\title{
Genetic and Biochemical Characterization of Ergot Alkaloid Synthesizing Fungi and their Symbionts
}

\author{
Matthew Duane Maust \\ West Virginia University, mmaust@mix.wvu.edu
}

Follow this and additional works at: https://researchrepository.wvu.edu/etd

Part of the Analytical Chemistry Commons, Environmental Microbiology and Microbial Ecology Commons, Genetics Commons, and the Plant Pathology Commons

\section{Recommended Citation}

Maust, Matthew Duane, "Genetic and Biochemical Characterization of Ergot Alkaloid Synthesizing Fungi and their Symbionts" (2020). Graduate Theses, Dissertations, and Problem Reports. 7885.

https://researchrepository.wvu.edu/etd/7885

This Dissertation is protected by copyright and/or related rights. It has been brought to you by the The Research Repository @ WVU with permission from the rights-holder(s). You are free to use this Dissertation in any way that is permitted by the copyright and related rights legislation that applies to your use. For other uses you must obtain permission from the rights-holder(s) directly, unless additional rights are indicated by a Creative Commons license in the record and/ or on the work itself. This Dissertation has been accepted for inclusion in WVU Graduate Theses, Dissertations, and Problem Reports collection by an authorized administrator of The Research Repository @ WVU.

For more information, please contact researchrepository@mail.wvu.edu. 
Matthew Duane Maust

Follow this and additional works at: https://researchrepository.wvu.edu/etd

Part of the Analytical Chemistry Commons, Environmental Microbiology and Microbial Ecology Commons, Genetics Commons, and the Plant Pathology Commons 


\section{GENETIC AND BIOCHEMICAL CHARACTERIZATION OF ERGOT ALKALOID SYNTHESIZING FUNGI AND THEIR SYMBIONTS}

\section{Matthew Maust}

Dissertation submitted to the Davis College of Agriculture, Natural Resources and Design at West Virginia University in partial fulfillment of the requirements for the degree of

Doctor of Philosophy in

Genetics and Developmental Biology

Daniel Panaccione, Ph.D., Chair

Matthew Kasson, Ph.D.

F. Heath Damron, Ph.D.

Werner Geldenhuys, Ph.D.

Division of Plant and Soil Sciences

Morgantown, West Virginia

2020

Keywords: ergot alkaloids, mycotoxins, fungi, mass spectrometry, specialized metabolites 


\title{
ABSTRACT \\ Genetic and biochemical characterization of ergot alkaloid synthesizing fungi and their symbionts
}

\begin{abstract}
Matthew Maust
Ergot alkaloids are fungal tryptophan derived toxins which affect mammalian circulation and neurotransmission. These compounds are biosynthesized by a conserved genetic pathway, known as the ergot alkaloid synthesis (EAS) pathway by fungi belonging to the ascomycete families Trichocomaceae and Clavicipitaceae. Several Ipomoea species and related plants in the morning glory family harbor vertically transmitted symbiotic fungi in the genus Periglandula, also members of Clavicipitaceae, that produce ergot alkaloids. Metabolomic analysis of seeds identified a previously uncharacterized glycoside form of the pharmaceutically important ergot alkaloid, ergonovine. Several species belonging to the fungal genus Metarhizium have recently been shown to have the capacity to express lysergic acid derived compounds. Metarhizium species are prolific entomopathogens and have the capacity to form beneficial relationships with plants by colonizing their roots. Proteomics analysis showed that wildtype and knock out strains of Metarhizium brunneum infected insects had different antimicrobial peptide and protein expression profiles based on the presence of ergot alkaloids. Metabolomics analysis found that unlike with insects, $M$. brunneum does not produce ergot alkaloids when grown in conjunction with plants and factors known to promote microbial symbiosis and stress-response in plants were upregulated. Fungi from Trichocomaceae (genera include Penicillium) diverge from fungi in Clavicipitaceae at a middle step of the ergot alkaloid synthetic (EAS) pathway to produce fumigaclavines and related compounds. Penicillium biforme is a known producer of rugulovasine A/B, which has never been observed in Penicillium camemberti. Data presented here suggest that the ancestor of modern $P$. camemberti had the capacity to synthesize rugulovasines and other ergot alkaloid precursors but lost this capability due to a V13G mutation on the protein. Analysis of the genomes from $P$. camemberti and $P$. biforme revealed that the two species contain the same cluster of EAS genes, and both organisms express mRNA from these genes in specific culture conditions. Metabolomics analysis confirmed that the regulatory elements needed for EAS gene expression are functional in $P$. camemberti. These results show how genetic techniques and biochemical analysis can provide new insights into these organisms.
\end{abstract}




\section{Table of Contents}

CHAPTER 1: INTRODUCTION

CHAPTER 2: DISCOVERY OF A NOVEL ERGOT ALKALOID GLYCOSIDE FROM METABOLOMIC ANALYSIS OF IPOMOEA SPECIES

$\begin{array}{lr}\text { ABSTRACT } & 8\end{array}$

$\begin{array}{lr}\text { INTRODUCTION } & 10\end{array}$

METHODS 12

$\begin{array}{lr}\text { RESULTS } & 18\end{array}$

$\begin{array}{ll}\text { DISCUSSION } & 23\end{array}$

$\begin{array}{ll}\text { ACKNOWLEDGMENTS } & 25\end{array}$

$\begin{array}{ll}\text { FIGURES } & 27\end{array}$

CHAPTER 3: THE IMPACT OF ERGOT ALKALOIDS ON METARHIZIUM BRUNNEUM SYMBIOSIS WITH \begin{tabular}{ll}
\hline INSECTS AND PLANTS & 41 \\
\hline
\end{tabular}

$\begin{array}{ll}\text { ABSTRACT } & 41\end{array}$

$\begin{array}{ll}\text { INTRODUCTION } & 43\end{array}$

$\begin{array}{lc}\text { METHODS } & 44\end{array}$

$\begin{array}{ll}\text { RESULTS } & 49\end{array}$

DISCUSSION

$\begin{array}{ll}\text { FIGURES } & 56\end{array}$

CHAPTER 4: INVESTIGATION OF EASC AND EXPRESSION OF THE ERGOT ALKALOID SYNTHESIS

\begin{tabular}{lc} 
PATHWAY IN PENICILLIUM CAMEMBERTI & 68 \\
\hline
\end{tabular}

$\begin{array}{lc}\text { ABSTRACT } & 68\end{array}$

$\begin{array}{ll}\text { INTRODUCTION } & 70\end{array}$

$\begin{array}{ll}\text { METHODS } & 72\end{array}$

$\begin{array}{ll}\text { RESULTS } & 75\end{array}$

$\begin{array}{ll}\text { DISCUSSION } & 78\end{array}$

$\begin{array}{lr}\text { FIGURES } & 81\end{array}$

\begin{tabular}{lr} 
CHAPTER 5: SUMMARY & 93 \\
\hline
\end{tabular}

\begin{tabular}{ll} 
REFERENCES & 96 \\
\hline
\end{tabular} 


\section{CHAPTER 1 \\ Introduction}

Since ancient times, humans have had mostly negative interactions with the class of mycotoxins now known as ergot alkaloids. Humans have a long history of interaction with ergot alkaloids with regards to agriculture. Claviceps purpurea, a pathogen of rye, has been implicated in the contamination of livestock feed and food for humans. As a result of consumption, mammals typically experience conditions ranging from psychoactive effects, such as hallucination, to impacts related to the toxins' vasoconstrictive properties such as gangrene. Therefore, many countries monitor harvested grains for the presence of fungal structures associated with ergot alkaloid production (Belser-Ehrlich et al., 2013). In more recent times these compounds have been harnessed in medicine. This group of specialized metabolites have a commonality in that they are tryptophan-derived indoles, and share structural similarities with the mammalian signaling molecules serotonin, dopamine and noradrenaline (Wallwey \& $\mathrm{Li}, 2011)$. Because of this, ergot alkaloids have found a role as pharmaceutical agents for a number of human conditions, such as migraines, Parkinson's disease and to control bleeding during childbirth (Bonuccelli et al., 2009; Howard et al., 1964; P. C. Tfelt-Hansen \& Koehler, 2008).

These compounds are biosynthesized by a conserved fungal genetic pathway, known as the ergot alkaloid synthesis (EAS) pathway. Early steps in the pathway are highly conserved, with later steps employing different enzymes based on the species, leading to the diversity of structures that are known (Gerhards et al., 2014). Pathway intermediates, side-products and end-products can all be commonly observed in any particular synthesizing fungus, as the pathway is known to be relatively inefficient (Panaccione, 2011). Due to this diversity and inefficiency, a lot of work has been performed to understand what genetic and regulatory elements are involved in the production of the ergot alkaloid suite found in any fungus or fungal host. 
The ergot alkaloid synthesis pathway is composed of an entire gene cluster and can be found in several Ascomycete families including Clavicipitaceae and Trichocomaceae (Robinson \& Panaccione, 2015). The metabolic pathway with gene names can be seen in figure 1. Early steps of the pathway are highly conserved, with the genes $d m a W$, easF, eas $C$, and eas $E$ found in representatives from both clades. The gene dmaW starts synthesis with the precursor dimethylallyltryptophan (DMAT), and the early pathway concludes with the production of chanoclavine-I by the eas $C$ gene.

Most species of ergot alkaloid synthesizing organisms proceed through a couple of transitional steps with enzymes encoded by easA and easG, leading to the production of festuclavine, or the related compound agroclavine. It is at this point that major differences in end-products start to occur. A large proportion of these variations are found within the Clavicipitaceae family. These compounds can be classified as clavines, lysergic acid amides and ergopeptines (Florea et al., 2017). Species representing this clade include Periglandula ipomoeae, Metarhizium brunneum, and Epichloë inebrians. A separate group of modifications to the ergot alkaloid synthesis pathway, the fumigaclavines, occurs in a group of fungi from the family Trichocomaceae. Representatives from this family include Penicillium camemberti, Penicillium biforme, and Neosartorya fumigata. The production and effects of the ergot alkaloids produced by $P$. ipomoeae, $M$. brunneum and $P$. biforme (and the lack thereof by $P$. camemberti) form the basis for study for the interactions investigated in this work. These compounds are utilized by these fungi to provide competitive advantages in their respective niches.

Many ergot alkaloid producing fungi occupy a role as endophytes of the plants they grow within. These fungi provide their plant hosts with a competitive advantage, and encourage them to maintain the relationship, by producing ergot alkaloids which punish or even outright prevent herbivory. Lambs grazing on ergot alkaloid containing pastures show signs of toxicosis and end up with lower body mass gains when compared to those grazing on ergot alkaloid free pastures (Parish et al., 2003a). A similar impact is observed in steers grazing on ergot alkaloid containing pastures (Parish et al., 2003b). This 
study also showed a behavioral component, where ergot alkaloid impacted steers had a decrease in grazing habits as well as water consumption. This effect was also observed in rabbits, and was specific to the presence of ergot alkaloids (Panaccione et al., 2006). In this study, the rabbits dramatically preferred feeding on plants that contained endophytes but lacked ergot alkaloids versus plants that lacked endophytes. When ergot alkaloids accumulated in the grass, this effect was counteracted. Satiety was also evaluated in this same study, which showed that the ergot alkaloid ergovaline reduced rabbit appetites.

Ergot alkaloids also provide plants with a competitive advantage with regards to challenge from invertebrates, including insects and nematodes. The ergot alkaloid content of the endophyte infected Cloudcroft sleepygrass (Achnatherum robustum) protected these plants from aphids (Shymanovich et al., 2014). This effect was confirmed when oat leaves were supplemented with the ergot alkaloid ergonovine. Another study showed that ergot alkaloids contribute to caterpillar resistance by endophyte infected ryegrass (Potter et al., 2008). Ergot alkaloid containing morning glories also gain an advantage against the insect pest potato psyllid (Bactericera cockerelli). In one study it was observed that morning glory species which contained large quantities of ergot alkaloids killed the insects rapidly, whereas they survived to adulthood on the species which lacked ergot alkaloids (Kaur et al., 2018). In a recently published follow-up study, it was shown that even supplementing endophyte lacking leaves with even a small amount of ergot alkaloids was enough to cause a large and significant increase in psyllid mortality (Kaur et al., 2020). In another study involving morning glory roots, ergot alkaloids were shown to have a protective effect when the plants were grown in soil infested with Southern root-knot nematode (Meloidogyne incognita) (Durden et al., 2019).

Ergot alkaloids also help fungi compete directly as they cause infections or compete for nutrients. Waxworm larvae (Galleria mellonella) which were injected with ergot alkaloid deficient strains of Neosartorya fumigata had significantly lowered mortality rates. Fumigaclavine $\mathrm{C}$, the pathway 
endpoint, appears to be a particularly important virulence factor, as strains of the fungus accumulating earlier pathway intermediates had lower virulence (Panaccione \& Arnold, 2017). Another study involving the entomopathogen Metarhizium brunneum, showed that this fungus produces high levels of ergot alkaloids when it infects living waxworm larvae (Leadmon et al., 2020). Perhaps ergot alkaloids are used as a virulence factor by the fungus when causing infection. Alternatively the compounds may be used to help the fungus secure nutritional resources in competition with other microorganisms, as some ergot alkaloids have been shown to have antibacterial properties (Eckart \& Pertz, 1999).

As a result of the diversity of structures found in ergot alkaloid synthesis pathways, and due to the inefficiency of the pathway leading to relatively high-level accumulation of pathway intermediates, metabolome profiling is a powerful approach to studying these fungi. Apart from ergot alkaloids, many fungi carry in their genomes the capacity to synthesize a whole suite of specialized metabolites which play roles in human activities including agriculture, production and preservation of food, and medicine (Nisa et al., 2015). With the development of accurate mass, high resolution mass spectrometers with increasingly fast scanning speeds, experiments which profile the global metabolome of a given organism or symbiotic relationship have become possible (Schrimpe-Rutledge et al., 2016).

While molecular biology and proteomic approaches can be utilized in powerful ways to study fungal gene regulation and phenotypes, many fungi lack the characterized genomes which help enable these types of analysis. Small molecule metabolite profiling becomes an elegant solution for investigating these situations. Where a genome may include $>20,000$ genes and the resulting proteome may result in $>100,000$ potential proteins, a typical metabolome may only contain approximately 5,000 species (Schrimpe-Rutledge et al., 2016). This issue is further compounded when fungi are involved in symbiotic relationships (e.g. plant symbiosis, insect pathogenesis) or are studied in complex matrices (e.g. milk for cheese making). Fortunately, while this large variation in genome and proteome can lead to high levels of complexity, these same proteins and genes contribute to the function of a large 
proportion of highly conserved pathways (Peregrín-Alvarez et al., 2009). The result is that variability in the abundance (or presence/absence) of a relatively smaller number of metabolites can be linked back the gene expression profiles. These metabolite profiles constitute the observations of phenotypes which relate to the underlying genotypes by which they are produced and can ultimately shed light into the nature of interactions between fungi and their hosts or their environment.

The production and effects ergot alkaloids form the basis for study for the interactions investigated in this work.

\section{Objectives for the work discussed in this document:}

1. To investigate the impacts of Periglandula species infections on the host metabolomic pathway expression of plants belonging to the genus Ipomoea

a. While many species of morning glories harbor fungal symbionts from the genus Periglandula, morning glories can be "cured" of fungus. While these "cured" plants seem to grow normally without their symbiont it is unknown how the metabolism of the plants changes in response to the fungus or ergot alkaloids.

2. To evaluate the metabolite profile of Ipomoea sp. for biomarkers indicating presence of nonergot alkaloid producing fungi

a. Ergot alkaloids can be detected in large number of species in the Convolvulaceae family and are used as a biomarker to indicate the presence of Periglandula sp. symbionts. That Periglandula sp. cannot be grown in pure culture makes studying these organisms difficult and has confounded attempts at generating a full genome sequence. It is unknown if there are species of Periglandula, which are symbionts of morning glories which lack that ability to produce ergot alkaloids. 
3. To investigate the impact ergot alkaloid expression by Metarhizium brunneum has on the immune response of its insect hosts

a. Fungi belonging to the genus Metarhizium are well characterized as entomopathogens. They are known to secrete a number of proteins and specialized metabolites which assist in colonizing insects. M. brunneum was recently shown to synthesize a suite of ergot alkaloids, it is unknown what role these play in virulence.

4. To investigate how plant metabolite expression changes as a response to M. brunneum symbiosis

a. M. brunneum can grow as symbiont of plants, as a result it is used as an insect biocontrol agent for several crops. Since these fungi may alter expression of host metabolites, it is important to understand the expression of host metabolites in these conditions.

5. To investigate the biochemical mechanism(s) which prevent Penicillium camemberti from producing the ergot alkaloids predicted by the genes shared with its ergot alkaloid producing ancestor, Penicillium biforme.

a. Like its close relative, P. biforme, P. camemberti transcribes genes from its EAS pathway under certain conditions. Despite this fact, the rugulovasines produced by $P$. biforme are not detected in cultures in $P$. camemberti.

b. Neither P. biforme nor $P$. camemberti produce mRNA transcripts for EAS genes, or the ergot alkaloid profiles predicted by their genes, when grown in cheese making conditions. 

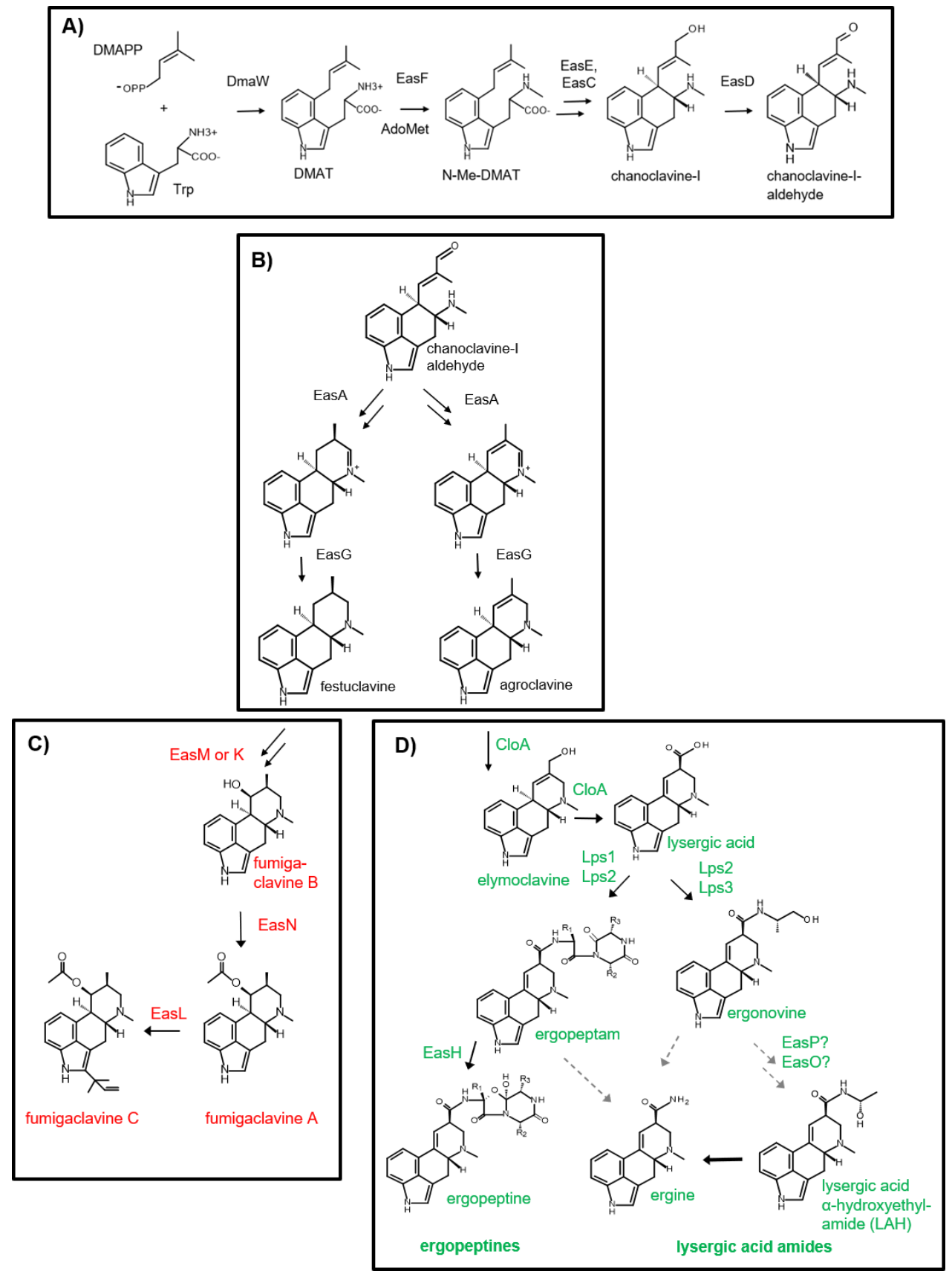

Figure 1. A map showing ergot alkaloid synthetic pathways. A) Highly conserved early precursor metabolites, B) after the middle steps, structures diverge according to lineage with those found in C) Trichocomaceae and those found in D) Clavicipitaceae. Redrawn from Robinson and Panaccione (2015). 


\section{CHAPTER 2 \\ DISCOVERY OF A NOVEL ERGOT ALKALOID GLYCOSIDE FROM METABOLOMIC ANALYSIS OF IPOMOEA SPECIES}

\section{Abstract}

Ergot alkaloids are fungal tryptophan derived toxins that affect human circulation and neurotransmission. Several Ipomoea species and related plants in the morning glory family harbor vertically-transmitted, symbiotic fungi in the genus Periglandula that produce ergot alkaloids. Many additional Ipomoea species are found to contain ergot alkaloids, indicating symbiosis with uncharacterized Periglandula species. A metabolomics approach was used to investigate biomarkers of fungal infection, which could indicate the presence of cryptic non-ergot producing Periglandula species. The metabolites of the Ipomoea tricolor seeds studied were collected from Periglandula sp.-infected plants $(\mathrm{P}+)$ or plants that had been cured by treatment with fungicide $(\mathrm{P}-)$. Seed extracts were screened for ergot alkaloids by fluorescence HPLC (LC-FLD), and total metabolites by mass spectrometry. Previously reported ergot alkaloids were present in high concentrations in P+ seeds and were not detected in P-seeds. Amino acid concentrations and detected plant stress hormones did not differ significantly between treatments. Analytes that were significantly more abundant in P+ seeds compared to P-seeds were compared to metabolomes from seed extracts of ergot alkaloid-positive and ergot alkaloid-negative seeds of Ipomoea parasitica and Ipomoea pes-caprae, as well as from nine ergot alkaloid-deficient Ipomoea species. Four metabolites tracked the presence of symbiont in this survey. One of these compounds identified by MS/MS analysis is a previously uncharacterized glycoside of ergonovine, a pharmaceutically important compound. The data indicate that apart from the 
accumulation of ergot alkaloids, Periglandula species have a minimal impact on the metabolome of seeds of their host plants. Furthermore, I found no evidence of cryptic, non-ergot alkaloid producing Periglandula species in seeds of the nine additional Ipomoea species analyzed. 


\section{$\underline{\text { Introduction }}$}

Ergot alkaloids are a class of specialized metabolites biosynthesized from tryptophan by a largely conserved gene pathway expressed in fungi (Robinson \& Panaccione, 2015). This class of compounds has a well-documented history of toxicity to invertebrates and mammals (Florea et al., 2017). Perhaps owing to their capacity to synthesize these compounds, Clavicipitaceous fungi occupy a variety of ecological niches. For members of the genus Metarhizium this takes the role of a pathogen of invertebrates (St. Leger et al., 2011). Within the genera Epichloë and Claviceps this manifests in a symbiotic relationship with monocotyledonous plants from the order Poales. One thing these genera all have in common, apart from their reliance on host organisms, is the expression of ergot alkaloids.

For years, it had been thought that the expression of ergot alkaloids was not unique to fungi, with certain plants within the family Convolvulaceae also accumulating these compounds. The potential source of these biosynthetic pathways may have been convergent evolution or acquisition through horizontal gene transfer from plant to fungi or vice versa (Steiner \& Leistner, 2012). Unexpectedly, experimentation revealed these plants accumulating ergot alkaloids were infected with a Clavicipitaceous fungi of the genus Periglandula that were the source of the ergot alkaloids (Steiner et al., 2011). There were only two signs of the infection - the presence of ergot alkaloids and, in some species of Ipomoea, white fungal colonies on the surface of new unfolded leaves. Curing the plant of the fungus by fungicide treatment eliminated the ergot alkaloids along with the fungus (Kucht et al., 2004). Much of the biochemistry of the symbiosis is a mystery. It is unknown how Periglandula species infecting plants in the genus Ipomoea impact pathway expression of the host plant and whether the plant responds to the presence of the fungus as it would a pathogen. Furthermore, while it is known that ergot alkaloid expression in morning glories always accompanies infection by a Periglandula 
species, it is unknown if there are non-ergot alkaloid producing fungi from that genus which could be present.

While some DNA sequencing has been performed on Periglandula species expressing ergot alkaloids, it is unknown if the symbiosis results in changes to plant or fungal gene expression. The biology of fungi in the genus Periglandula and their inability to be grown in pure culture has made genome sequencing challenging. A lack of a well-populated database from which to work from make RNA sequencing based approaches challenging and liquid chromatography mass spectrometry (LC-MS) proteomics based approaches impossible. Furthermore, the inability to grow Periglandula in pure culture makes distinction between plant and fungal genes or proteins dubious.

In recent years, developments in mass spectrometry and chromatography have enabled researchers to quantitatively profile thousands of small molecule metabolites simultaneously from a single sample. Stationary phases used in hydrophilic interaction chromatography (HILIC) allow for the reversal of mobile phases typically seen in a reversed phase chromatography gradient, allowing for improved retention of extremely polar compounds (Buszewski \& Noga, 2012). While these compounds can be challenging to separate chromatographically, high resolution accurate mass spectrometry (HRAM), found in quadrupole time of flight (QTOF) and quadrupole orbitrap mass spectrometers, allows for improved detection of molecules within a given range of mass channels when compared to older unit mass resolution instruments. With these techniques, individual isotopes from co-eluting compounds can be distinguished, and their chromatographic features can be resolved (Lesur \& Domon, 2015). As a result of these improvements, liquid chromatography mass spectrometry metabolomic biomarker discovery based approaches have found acceptance in clinical diagnostic and drug discovery arenas (Nagy-Szakal et al., 2018; Yeung, 2018). 
By leveraging the technological advances enabling HILIC-QToF mass spectrometry, the objective for this study was to investigate the impacts of Periglandula species infections on the host metabolomic pathway expression of plants belonging to the genus Ipomoea. Furthermore, since it is unknown whether non-ergot alkaloid expressing fungi are present in Ipomoea infections, biomarkers indicating fungal presence were also evaluated.

\section{Methods}

\section{Biological materials and metabolite extractions}

Seeds from Periglandula sp.-infected $(\mathrm{P}+)$ Ipomoea tricolor cv. Pearly Gates and cv. Flying Saucers as well as seeds that were cured of fungus by treatment with fungicide ( $\mathrm{P}-$ ) were acquired from the Keith Clay lab (Indiana University, Bloomington, Indiana). The P+ and P-seeds had been collected from plants demonstrated to contain or be free of Periglandula fungus (which has not yet been formally described; Beaulieu et al., 2013). This study made use of an extensive collection of methanolic extracts of seeds of Ipomoea species collected from a world-wide sampling of herbarium samples and wild plants by Keith Clay and students(Beaulieu, 2014). Previously prepared ergot alkaloid extractions from Ipomoea species (I. parasitica, I. acanthi, I. barbatisepala, I. clavata, I. pes-caprae, I. pandarata, I. imperati, I. aquatica, I. tubiodes, I. carnea, I. polpha, I. leptophylla) were weight normalized for further metabolomic analysis.

Methanol extracts of pulverized seeds from I. leptophylla and I. tricolor were screened for ergot alkaloids by high resolution accurate mass LC-MS/MS analysis. A seed was placed inside a 1-mL screw cap tube containing 10 glass beads, and tubes were shaken on a Fastprep 120 instrument (Bio101, Carlsbad, CA) at $6 \mathrm{~m} / \mathrm{sec}$ for 20 seconds. Bead beating was repeated after a brief cooling period if necessary, to pulverize the seed. The seed powder was incubated at room temperature for 10 minutes 
with $500 \mu \mathrm{L}$ of HPLC grade methanol. Following incubation, solids were pelleted by centrifugation and the supernatant was removed.

Polar metabolite extraction followed a similar workflow. Metabolite extracts were weight normalized based on the weight of the intact seeds. The metabolite extraction solvent $(50 \%$ methanol, $50 \%$ water) was added to yield a concentration of $10 \mathrm{mg}$ of seed per $1 \mathrm{~mL}$ of extraction solvent. After bead beating, samples were centrifuged to pellet insoluble material, and the supernatants were removed. The supernatants were stored at $-80^{\circ} \mathrm{C}$ until analyzed.

\section{QToF Metabolomics}

Polar metabolites were separated using HILIC chromatography, by injecting $10 \mu \mathrm{L}$ of extracts onto a 2.1 x 100 mm, $5 \mu \mathrm{m}$ Phenomenex Luna NH2 column (Torrance, CA). A 15 minute linear gradient, which ramped from $90 \%$ solvent $B(100 \%$ acetonitrile) to $60 \%$ solvent $A$ (10 mM ammonium acetate) at a flow rate of $300 \mu \mathrm{L} / \mathrm{min}$, was used to elute the metabolites for detection by electrospray ionization mass spectrometry. Each sample was analyzed in positive and negative ionization modes. The LC-MS system used for analysis consisted of an Infinity 1290 ultra-high pressure liquid chromatography instrument (Agilent Technologies, Santa Clara, CA) coupled to an Agilent 6530 quadrupole time of flight (QToF) mass spectrometer configured with a jet stream source for electrospray ionization. For both positive and negative ionization modes, the mass spectrometer scanned over a range from 50 to 1200 $\mathrm{m} / \mathrm{z}$ with a scan speed of $2 \mathrm{hz}$. 


\section{Analysis of Metabolomics Data}

LC-MS data were processed using Agilent Masshunter Profinder (version B.06.00) in batch mode according to polarity of acquisition. Chromatographic features were extracted which contained a minimum peak height of 600 counts and a maximum charge state of 2 . The resulting chromatography was inspected for consistency between samples, with retention time and mass alignments performed on irregular features. Peak areas from these chromatographic features were exported to Mass Profiler Professional (version 2.4.3) for further filtering, quality control and statistical analysis. Differences in peak abundances of chromatographic features between ergot alkaloid containing seeds and ergot alkaloid lacking seeds (as determined by HPLC with fluorescence detection) were assessed by a Bonferonni adjusted T-test $(p<0.05)$, with a minimum fold change $>5$.

\section{Regarding Mass Spectrometry methods}

A variety of instrument platforms were utilized during the course of this research. A distinction could be made between high resolution accurate mass instruments, such as the Agilent Technologies 6530 quadrupole time of flight mass spectrometer or the Thermo Scientific Q Exactive Orbitrap mass spectrometer, and unit mass resolution instruments (i.e. Thermo Scientific LCQDecaXP, Sciex QTrap 5500). The HRAM instruments both offer sub $10 \mathrm{ppm}$ mass measurements as well as efficient fragmentation mechanisms, which are conducive for the identification of small molecules (Ichou et al., 2014; Kind et al., 2018). Under ideal circumstances, all of the mass spectrometry analysis in this study would have been carried out on such instruments. Due to uncontrollable circumstances, analytical methodologies were adapted to the instruments which were available at the time of analysis. Some common threads linking these experiments were the usage of positive ion mode analysis, reversed phase chromatography and mobile phases (following the initial discovery of ergonovine glycoside on a 
HILIC chromatographic method). While precursor mass assignments from unit mass resolution instruments are not sufficient in and of themselves to confirm the identity of a peak, the selectivity afforded by retention times and tandem mass spectra were utilized to confirm the presence of the molecule(s) of interest throughout these experiments. Data in figures 2, 4, 5, and 8 were collected using a Thermo Q Exactive. Data in figure 3 were collected using an Agilent 6530 QToF. Data in 7a were collected using a Thermo LCQDecaXP, while 7b and 10 were collected using a Sciex QTrap 5500.

\section{Q Exactive analysis of ergot alkaloid extracts}

Reversed phase separations of analytes contained in the supernatant were performed by injecting $5 \mu \mathrm{L}$ onto a $2.1 \times 100 \mathrm{~mm}, 3.5 \mu \mathrm{m}$, Zorbax SB-C18 LC column (Agilent Technologies, Santa Clara, CA) held at $40^{\circ} \mathrm{C}$. Analytes were eluted with a gradient ramping from $95 \%$ solvent $A(0.1 \%$ formic acid) and $5 \%$ solvent $B(0.1 \%$ formic acid, acetonitrile) to $50 \%$ solvent B over 15 minutes, which was generated using an Accela 1290 UHPLC instrument (Thermo Scientific, San Jose, CA) at a flow rate of $300 \mu \mathrm{L} / \mathrm{min}$. Detection of analytes was performed by positive polarity electrospray ionization using an in-line $Q$ Exactive hybrid quadrupole Orbitrap mass spectrometer (Thermo Scientific, San Jose, CA), operated in a data dependent acquisition mode. A precursor scan of 200 to $600 \mathrm{~m} / \mathrm{z}$ was generated using the 70,000 resolution setting. The top five most abundant ions from the precursor scan were isolated by the quadrupole (2 Da isolation window) for higher-energy collisional dissociation (HCD) set at a normalized collision energy of 30. Fragment ions were then scanned with a resolution setting of 35,000. 


\section{LCQ Deca XP analysis of ergot alkaloid extracts}

Reversed phase separations of analytes contained in the supernatant were performed by injecting $5 \mu \mathrm{L}$ onto a $2.0 \times 150 \mathrm{~mm}, 4 \mu \mathrm{m}$, Synergi Polar-RP LC column (Phenomenex, Torrance, CA) held at $30^{\circ} \mathrm{C}$. Analytes were eluted with a gradient ramping from $86 \%$ solvent $\mathrm{A}(5 \%$ acetonitrile $+0.1 \%$ formic acid) and $14 \%$ solvent $B(0.1 \%$ formic acid $+75 \%$ acetonitrile) to $100 \%$ solvent $B$ over 20 minutes, which was generated using an Surveyor HPLC instrument (Thermo Scientific, San Jose, CA) at a flow rate of $200 \mu \mathrm{L} / \mathrm{min}$. Detection of analytes was performed by positive polarity electrospray ionization using an in-line LCQ Deca XP ion trap mass spectrometer (ThermoFinnigan, San Jose, CA), operated in data dependent acquisition mode. A precursor scan of 200 to $500 \mathrm{~m} / \mathrm{z}$ was generated using the 70,000 resolution setting. The top most abundant ion from the precursor scan was isolated by the quadrupole (2 Da isolation window) for collision induced dissociation (CID) set at a normalized collision energy of 35.

\section{Sciex 5500 QTrap analysis ergot alkaloid extracts}

Ergonovine and a previously unknown glycoside of ergonovine were monitored in plant extracts by positive mode electrospray ionization LC-QQQ-MS using a Sciex ExionLC AD UHPLC coupled to a Sciex QTrap 5500 linear ion trap quadrupole mass spectrometer (Sciex, Framingham, MA). Chromatographic separations were performed on $2.1 \times 50 \mathrm{~mm}, 3.5 \mu \mathrm{m}$, Zorbax SB-C18 column (Agilent Technologies, Santa Clara, CA) using a gradient composed of $0.1 \%$ formic acid in water (solvent $\mathrm{A}$ ) and $0.1 \%$ formic acid in acetonitrile (solvent B). The gradient ramped from $5 \%$ solvent B to $95 \%$ solvent B over 7 minutes, at a flow rate of $300 \mu \mathrm{L} / \mathrm{min}$, with the column held at $40^{\circ} \mathrm{C}$.

Mass spectrometry analysis was performed using multiple reaction monitoring. Ergonovine was monitored using the $326.3 \rightarrow 208.0$ transition. Ergonovine glycoside was monitored using the $488.3 \mathrm{~m} / \mathrm{z}$ 
$\rightarrow 326.3 \mathrm{~m} / \mathrm{z}$ transition. Analytes were fragmented using a dwell time of $50 \mathrm{msec}$ and a collision energy of 35 .

\section{Extraction of ergot alkaloids from drunken horse grass (Achnatherum inebriens)}

Three biological replicates of homogenized leaves of Epichloë gansuense-infected Achnatherum inebrians were received from the Chunjie Li lab (Lanzhou University, Lanzhou, Gansu Province, China). Homogenates $(50-100 \mathrm{mg}$ ) were incubated in $500 \mu \mathrm{L}$ of $50 \%$ HPLC grade methanol to extract ergot alkaloids. The supernatant was removed after centrifuging at $14,000 \times \mathrm{g}$ for 10 minutes to pellet solids.

\section{Plant incubations}

Leaves were removed from P+ and P- Ipomoea tricolor cv. Pearly Gates plants. The leaves were cut at the petiole, which was then submerged into a $2 \mathrm{~mL}$ microcentrifuge tube containing a $3 \mathrm{mg} / \mathrm{mL}$ solution of ergonovine (Sigma-Aldrich, St Louis, MO) in water. The tube was then covered with parafilm. The leaf was incubated in the solution for several days; ergonovine solution that was lost to transpiration by the leaf or by evaporation was replaced. Following this treatment, the leaf was then cut into $2 \times 2 \mathrm{~mm}$ pieces and bead beaten in a $50 \%$ methanol extraction solution (as described above for seeds).

Leaves were removed from plants listed in Table 2. All plants were acquired from the campus of West Virginia University or the surrounding area (Morgantown, WV). The leaves were cut at the petiole or approximately three inches from the end of the leaf (for plants lacking a petiole), which was then submerged into a $15 \mathrm{~mL}$ centrifuge tube containing a $3 \mathrm{mg} / \mathrm{mL}$ solution of ergonovine. The tube was then capped with a covering of paraffin film. Leaves were incubated in the solution for a week, ergonovine solution that was lost to transpiration by the leaf or by evaporation was replaced. Following 
this treatment, the leaf was then cut into $2 \times 2 \mathrm{~mm}$ pieces and bead beaten in a $50 \%$ methanol extraction solution.

\section{Method for PNPGal/UDP gal incubations with b-galactosidase}

Ergonovine and PNPGal/Glu or UDPGal/Glu (Sigma Aldrich, St. Louis, MO) were added to a solution containing phosphate buffered saline $\mathrm{pH} 7.5$ with $\mathrm{MgCl}(1 \mathrm{mM})$ and $\beta$-mercaptoethanol $(5 \mathrm{mM})$. Ergonovine and activated hexoses were added in equimolar amounts for the respective incubations, for a final concentration of $10 \mathrm{mM}$. The samples were incubated for 8 hours at room tempurature. A set of PNPGal incubations were repeated with the same buffer conditions ( $\mathrm{pH} 4.5$ and $\mathrm{pH} 6)$ and with or without boiling prior to incubation. Samples were analyzed using a Sciex 5500 Qtrap, LCQ DecaXP or Q Exactive, where noted in the results.

\section{$\underline{\text { Results }}$}

\section{Ergot alkaloid content of Ipomoea sp. seeds}

The infection status of Periglandula-infected ( $\mathrm{P}+$ ) Ipomoea tricolor seeds (cv. Pearly Gates) was confirmed by analyzing extracts for the same assortment of ergot alkaloids (chanoclavine-I aldehyde, chanoclavine, ergine, ergonovine, LAH) as previously reported (Beaulieu et al., 2015) (figure 1). Retention times, mass spectra and tandem mass spectra matched what is found in the related Metarhizium brunneum for these ergot alkaloids (Leadmon et al., 2020). Those I. tricolor seeds that had been cured of fungus by treatment with fungicide (P-) lacked all ergot alkaloids. Among the tested seeds from I. acantha, I. aquatica, I. carnea, I. clavata, I. imperati, I. pandurata, I. polpha and I. tuboides all individuals lacked ergot alkaloids. All seeds from I. barbatisepala and I. leptophylla contained ergot alkaloids (Beaulieu, 2014). In addition to the ergot alkaloids listed for I. tricolor, seeds of I. leptophylla 
also accumulated the on-pathway intermediate chanoclavine-I aldehyde to detectable levels (figure 2). Within the collection of seeds from I. pes-caprae, and I. parasitica different individuals contained and lack ergot alkaloids (Table 1). I. pes-caprae also accumulated ergobalansine, which agrees with the findings of Beaulieu et al. (2015).

\section{Metabolomic analysis of Ipomoea sp. seeds}

Full metabolite analysis was performed on methanol seed extracts by HILIC-LC-ESI-QTOF-MS. Samples were injected twice, for analysis in positive and negative ion modes. Metabolomic analysis tracked expression of 1768 compounds in negative ion mode, and 1371 compounds in positive ion mode. Analytes that were significantly more abundant, as assessed by t-test $(p<0.05)$ in $P+$ seeds compared to P-seeds were compared to metabolomes from seed extracts of ergot alkaloid-positive and ergot alkaloid-negative seeds of I. parasitica and I. pes-caprae, as well as from the nine ergot alkaloiddeficient Ipomoea species. Among some of the compounds that were identified, no detectable changes in the expression of cinnamate, amino acids, jasmonic acid and salicylic acid (often associated with response to microbial infection) were found between $\mathrm{P}+$ seeds and $\mathrm{P}$ - seeds. Four metabolites, with masses not associated with previously reported ergot alkaloids, tracked the presence of the Periglandula symbiont in this survey (figure 3). These data taken together, bolster the argument that there are no cryptic non-ergot alkaloid producing Periglandula symbionts of plants in the Ipomoea genus. In negative ion mode, three compounds - 551.2830 Da (putative formula: C26 H39 N3 07), 549.2759 Da (putative formula: C28 H33 N5 O4) and 581.2974 Da (putative formula: C24 H44 N O9 P) were identified as upregulated in P+ samples. In positive mode, a compound of 487.2389 Da (putative formula: C25 H33 N3 07) was found to be upregulated in P+ samples. For all four compounds, searches of precursor masses and ms/ms spectra using databases from Biocyc and Metlin, failed to produce identifications. 
Manual investigation of the $\mathrm{ms} / \mathrm{ms}$ spectra for $488.2391 \mathrm{~m} / \mathrm{z}$ (the $\mathrm{M}+\mathrm{H}$ ion of $487.2389 \mathrm{Da}$ ) revealed fragment ions of $326.1845 \mathrm{~m} / \mathrm{z}, 283.1428 \mathrm{~m} / \mathrm{z}, 223.1219 \mathrm{~m} / \mathrm{z}, 208.0747 \mathrm{~m} / \mathrm{z}$, and $197.1064 \mathrm{~m} / \mathrm{z}$. When compared to the fragmentation spectra of an authentic ergonovine analytical standard, these fragment $\mathrm{m} / \mathrm{z}$ values matched within a mass error of less than $1.0 \mathrm{ppm}$, indicating that the molecule contained ergonovine (figure 4). Investigation of the precursor mass minus the mass of the highest abundance fragment ion revealed a mass shift of $162.0523 \mathrm{Da}$, which is consistent with the mass of a hexose residue (-3.09 ppm mass error).

\section{Bioconversion capacity of $I$. tricolor tissues}

To test the requirement of the Periglandula fungus for bioconversion of ergonovine to the glycoside form, leaves from $\mathrm{P}+$ and $\mathrm{P}-\mathrm{I}$. tricolor plants were incubated in an ergonovine solution and evaluated by LC-MS. Initially, leaves from the P+ plants were evaluated, and bioconverted ergonovine to its glycoside form (figure 5). Conversion is evidenced by the two peaks of the $488 \mathrm{~m} / \mathrm{z}$ ion in the chromatograph. It is unknown whether these peaks are a result of different monosaccharides incorporated into the ergot alkaloid, or if these peaks correspond to different stereoisomers in the ergoline scaffold. Examples of chromatographic separation of such stereoisomers can be seen in figure 2 (e.g. LAH, ergine, ergonovine).

When compared with a non-incubated control, ergonovine levels in the leaf tissue increased, whereas ergonovine levels were below detectable limits in the non-incubated leaf sample. Likewise, it was found that ergonovine glycoside accumulated to detectable levels compared to the non-incubated control. Following this initial evaluation, a P- leaf was leaf was incubated in ergonovine, and ergonovine glycoside was detected. Tissue was collected from the root, leaf, stem and flower of a P- I. tricolor plant and homogenized in PBS, then incubated in the presence of ergonovine. While ergonovine was detected 
in the homogenates, ergonovine glycoside was observed at very low levels by HPLC with fluorescence detection (figure 1) but was below the limit of detection by mass spec (figure 6). These results indicate that the presence of the Periglandula symbiont is not required for ergonovine glycoside conversion, and that there is a structural component in the intact plant tissues which is required for efficient bioconversion to take place.

\section{Synthesis of ergonovine glycoside}

Prior findings from in vitro studies showed that ergonovine- $\beta$-D-galactoside (wherein ergometrine is a synonym for ergonovine) can be synthesized with $\beta$-galactosidase isolated from Aspergillus oryzae and p-nitrophenyl $\beta$-D-galactoside (PNPGal) as the galactose donor (Křen et al., 1990, 1992). In my study, these conditions were repeated using UDP-glucose (UDPGlu), UDP-galactose (UDPGal), PNPGal and p-nitrophenyl $\beta$-D-glucose (PNPGlu), to identify the individual peaks in the doublet for $488.2391 \mathrm{~m} / \mathrm{z}$ as seen in I. tricolor (figure 5). LC-MS analysis of UDPGlu incubations gave two prominent peaks of similar peak areas, eluting at 5.2 minutes and 6.9 minutes. In contrast, when the UDPGal incubation was analyzed, the second peak (6.9 minutes) showed a large increase in peak area relative to the peak at 5.2 minutes (figure 7). This result was confirmed with I. tricolor leaves, which also showed increased synthesis of the later eluting peak, when supplemented with UDPGal. An MRM assay revealed that the presence of UDPGal improved the glycosylation capacity of $I$. tricolor leaves (figure 8).

Furthermore, the data reveal a marked increase in the abundance of the later eluting peak when the plant leaf is incubated with UDPGal. This observation contrasts with the results of incubations with UDPGlu which did not produce an increased abundance for either peak (figure 9a). Epichloë inebriansinfected drunken horse grass samples analyzed along with the morning glory leaf UDP incubated samples revealed two peaks, matching the abundance profile (figure 9b). PNPGal and PNPGlu were also 
tested for in vitro enzymatic synthesis; these synthesis experiments yielded similar results to the UDPGal/Glu experiments. When $\beta$-galactosidase was deactivated with boiling prior to incubation, ergonovine glycoside was not detected (figure 9).

In both the PNP and UDP synthetic experiments, these data indicate that this enzymatic approach has a relatively low turnover in comparison to what was previously reported in in vitro studies both substrates were generated at a ratio of approximately 1:10 (glycoside to ergonovine) (Křen et al., 1990).

\section{Conversion capability of a variety of plants}

Ergonovine glycoside was observed in Periglandula containing Ipomoea species. Two species of plants from the Commelinidae clade (Achnatherum inebrians and Achnatherum robustum) harbor Epichloë. Representatives from this fungal genus are known to produce ergot alkaloids, including ergonovine. Sleepy grass (A. robustum) grows in the southwestern United States and is known to harbor two different types of ergot alkaloid producing fungal endophytes, an undescribed Epichloë sp. (ergonovine and ergine production) and Epichloë funkii (chanoclavine I production)(Shymanovich et al., 2014). Drunken horse grass (A. inebrians) grows in northwestern China and has also been shown to harbor ergonovine producing Epichloë inebrians. Both plants species from genus Achnatherum which contained ergonovine also contained ergonovine glycoside (figure 10), while the data are not shown it was observed that the chanoclavine I containing plants did not produce ergot alkaloid glycosides.

Since I. tricolor was found to convert ergonovine to ergonovine glycoside when the fungus is not present, plants representing a variety of clades were investigated for the capacity to perform this conversion. Plants were chosen from pteridophyta and ginkgoopsida (gymnosperm) to represent nonangiosperm clades. Plants were also chosen from the angiosperm clades Asteridae (Solanales and 
Asterales), Commelinidae, Dilleniidae, Hamamelididae, Liliidae, Magnoliidae and Rosidae. A full list of plants tested can be found in table 2. The MRM assay detected peaks corresponding to ergonovine glycoside from every representative tested. Plant specimens varied in the number of peaks which were detected and as well as in abundances. This may indicate variation in the types of sugar residues that were incorporated into the glycoside. Based on the results from the galactose incorporating experiments, many plants produced a peak corresponding to the retention time of ergonovine galactoside.

\section{Discussion}

The results presented indicate that apart from ergot alkaloids, Periglandula species have a minimal impact on the metabolome of seeds of their host plants. We also found no evidence of nonergot alkaloid producing Periglandula species in seeds of the Ipomoea species analyzed. However, it does appear that a biochemical process within the plant is in place which modifies the ergot alkaloid ergonovine to its glycoside form. As a result of this finding, the focus of this study shifted to explore the capacity for plants to perform this modification in vivo and characterize the structure of a newly discovered form of ergonovine.

The results presented here led to the proposed structure of the most abundant compound as ergonovine with a hexose residue conjugated at the primary hydroxyl group. This structure was originally described as ergonovine- $\beta$-D-galactoside by Křen et al (1992). They produced this compound in vitro by incubating PNPGal along with $\beta$-galactosidase from Aspergillus oryzae but never observed it from a natural source. Also, in this report Křen et al. synthesized galactosides of elymoclavine, 9,10dihydrolysergol, lysergol, and chanoclavine. An earlier report was made by Floss et al. (1976), who isolated elymoclavine-O- $\beta$-D-fructoside from a saprophytic culture of a Claviceps strain. In a related 
study, Claviceps purpurea grown in saprophytic culture media supplied with O- $\beta$-D-fructofuranosyl was shown to produce elymoclavine-O- $\beta$-D-fructosides (Flieger et al., 1989).

This present report differs from previous reports, in that ergonovine glycoside accumulated in vivo in the Ipomoea sp. symbiotic with Periglandula sp. that were evaluated in this study. Furthermore, as shown in this report, for ergonovine to be glycosylated in plant tissues a fungal symbiont is not required. Until now this phenomenon was only observed using fungal cultures induced with high levels of sugar containing substrates or in vitro with enzymes derived from fungal or bacterial origin. While Křen et al. (1992) reported generating glycosides from several forms of ergot alkaloids, of the ergot alkaloids produced by Periglandula sp., only ergonovine was glycosylated in vivo. This was the case across all the plants tested, which indicates that the enzymes required for this transformation have specificity for the chemical moieties found on ergonovine

The fragmentation pattern for the compound supports the presence of a hexose residue. Under low energy fragmentation (i.e. CID), it would be expected that a hexose residue connected via a glycosidic bond, would be readily dissociated from the parent ion(Yu et al., 2016). Indeed, the $326 \mathrm{~m} / \mathrm{z}$ ion representing ergonovine is the predominant ion found in the spectra. This evidence along with the accurate mass data, agree with the structure reported here, and by Křen et al. (1992). It is worth noting that the primary hydroxyl found on chanoclavine-I, the secondary hydroxyl on LAH, the carboxylic acid on lysergic acid, and the primary amine on ergine, apparently are not suitable sites for the catalytic activity of the enzyme responsible for this conversion in Ipomea sp., because glycosylated forms of these compounds were not detected. While many plant extracts contained multiple chromatographic peaks for ergonovine glycoside, the evidence suggests that one of the most abundant peaks is the galactoside form. Based on what can be found in other complex carbohydrates, it could be speculated that other hexose residues could alternately be incorporated into the structure. Perhaps other hexose residues which were not tested (e.g. mannose, fructose, etc.) could be responsible for other peaks present in the 
chromatographs presented. The modification of ergonovine also seems to be specific to hexose incorporation, as glucuronic acid forms were not observed.

The appearance of ergonovine glycoside in plant tissues is enzyme mediated and not a spontaneous reaction. While the data are not presented here, the one animal model tested (Galleria mellonella) did not produce ergonovine glycoside when infected with an ergonovine producing fungus. This process is possibly not specific to plants (it was synthesized in vitro using a fungal enzyme and galactose; Křen et al., 1992), however glycosyltransferase function is conserved across a wide range of plant clades. While performing the plant transformation experiments, vascular tissue seemed to be important for conversion. When these structures were disrupted or not enough ergonovine solution was provided to vascular tissue, conversion was reduced or halted. One explanation for the conservation of this function could be as a mechanism for mobility within plant tissue, as part of a detoxification process or defense response against ergot alkaloids. Plants are known to use glycosyltransferases for moving specialized metabolites throughout tissues or for storage (J. Wang \& Hou, 2009). This has been shown specifically with I. tricolor, where ergot alkaloid species are differentially allocated throughout the plant, independent of the presence of the fungal symbiont (Beaulieu et al., 2013). In Ipomoea sp. this may be a part of leveraging the symbiosis with its Periglandula symbiont. Most recently, it has been that observed the ergot alkaloids being transported throughout morning glories are a part of the defense response against nematodes (Durden et al., 2019), perhaps specialized glycosyltransferase enzymes are involved in transporting ergonovine in its glycoside form, from where it is produced by the fungus, to the infected roots.

\section{Acknowledgments}


Experiments with industrial hemp were conducted under License No. 0005 from the West Virginia

Department of Agriculture. Angie Macias was instrumental in identifying and collecting leaves from the variety of plants used in this work. 


\section{$\underline{\text { Figures }}$}

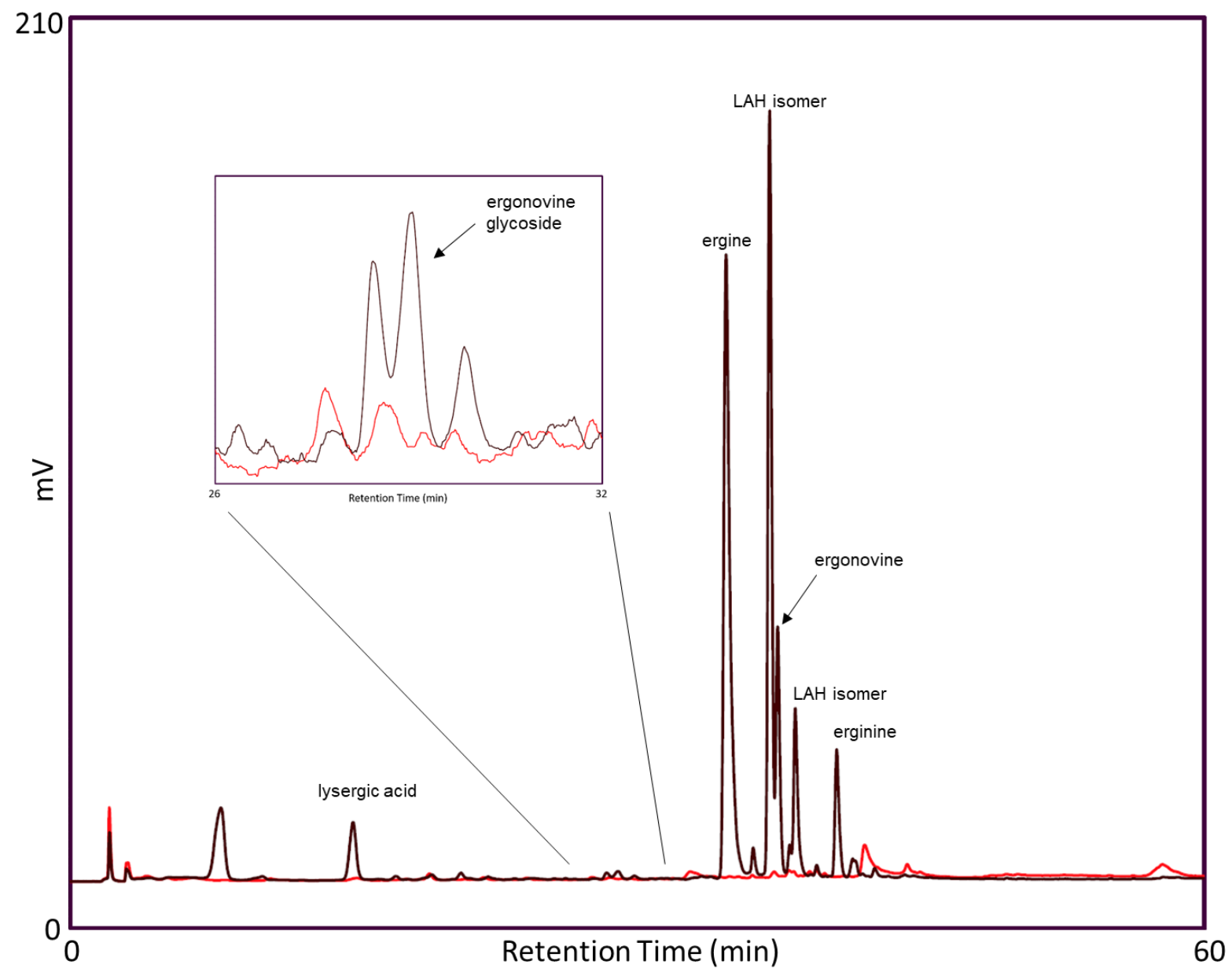

Figure 1. HPLC characterization of ergot alkaloids present in Ipomoea tricolor infected with Periglandula sp. endophyte (brown trace), or without endophyte (red). Ergot alkaloids were detected with fluorescence excitation at $310 \mathrm{~nm}$ and emission at $410 \mathrm{~nm}$. This ergot alkaloid profile matches what was previously observed in this symbiotic relationship (Beaulieu et al., 2013). 


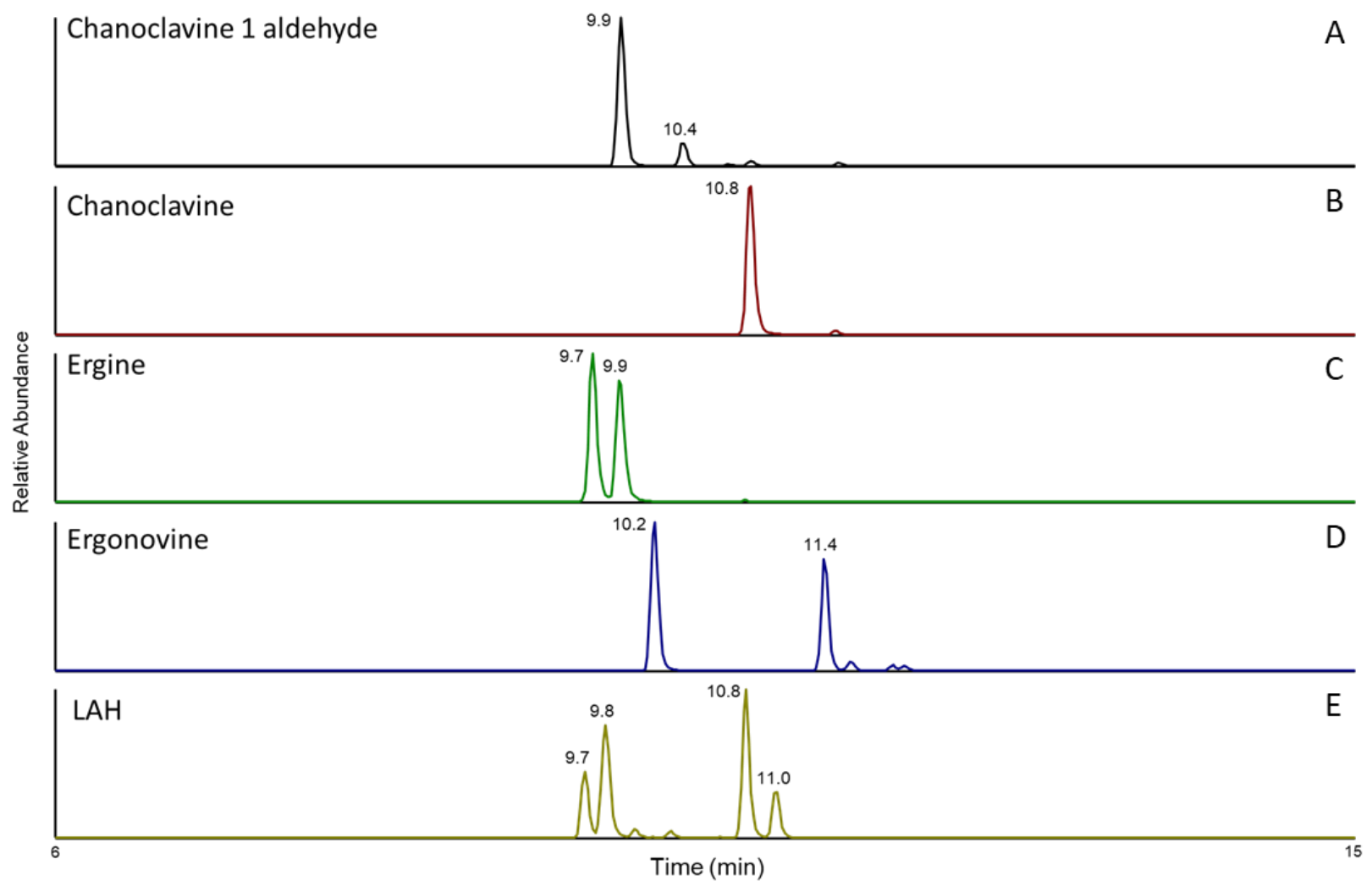

Figure 2a. Extracted ion chromatography, from a Q Exactive, of ergot alkaloids observed in I. leptophylla.

(A) chanoclavine 1 aldehyde, $255.1492 \mathrm{~m} / \mathrm{z}$, (B) chanoclavine, $257.1649 \mathrm{~m} / \mathrm{z}$, (C) ergine, 268.1445, $\mathrm{m} / \mathrm{z}$, (D) ergonovine, $326.1863 \mathrm{~m} / \mathrm{z}$, (E) D-lysergic acid $\alpha$-hydroxyethylamide (LAH), $312.1707 \mathrm{~m} / \mathrm{z}$. All ions were extracted with a $10 \mathrm{ppm}$ mass window centered on the theoretical monoisotopic $\mathrm{M}+\mathrm{H}$. The multiple peaks observed in ergine, ergonovine and LAH are chromatographically resolved stereoisomers. 


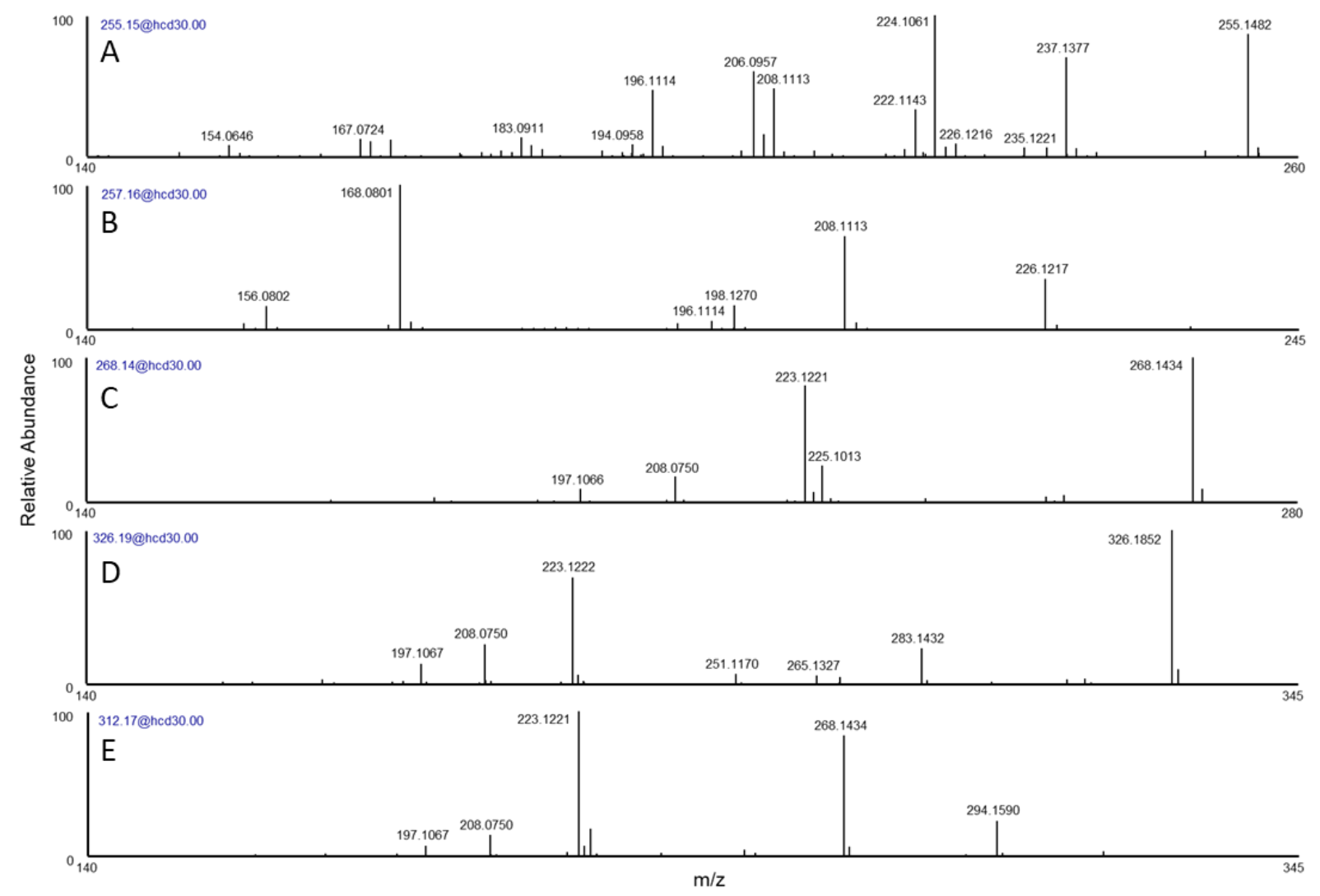

Figure $\mathbf{2 b}$. High resolution, accurate mass HCD fragmentation spectra, from a $Q$ Exactive, for ergot alkaloids detected in I. leptophylla. (A) chanoclavine 1 aldehyde, (B) chanoclavine, (C) ergine, (D) ergonovine, (E) LAH. Early pathway intermediates (A, B) show common fragment ions $-196.111 \mathrm{~m} / \mathrm{z}$, $208.111 \mathrm{~m} / \mathrm{z}$ and $226.122 \mathrm{~m} / \mathrm{z}$, while later pathway intermediates (C-E) show common fragment ions $197.107 \mathrm{~m} / \mathrm{z}, 208.075 \mathrm{~m} / \mathrm{z}$, and $222.122 \mathrm{~m} / \mathrm{z}$. 

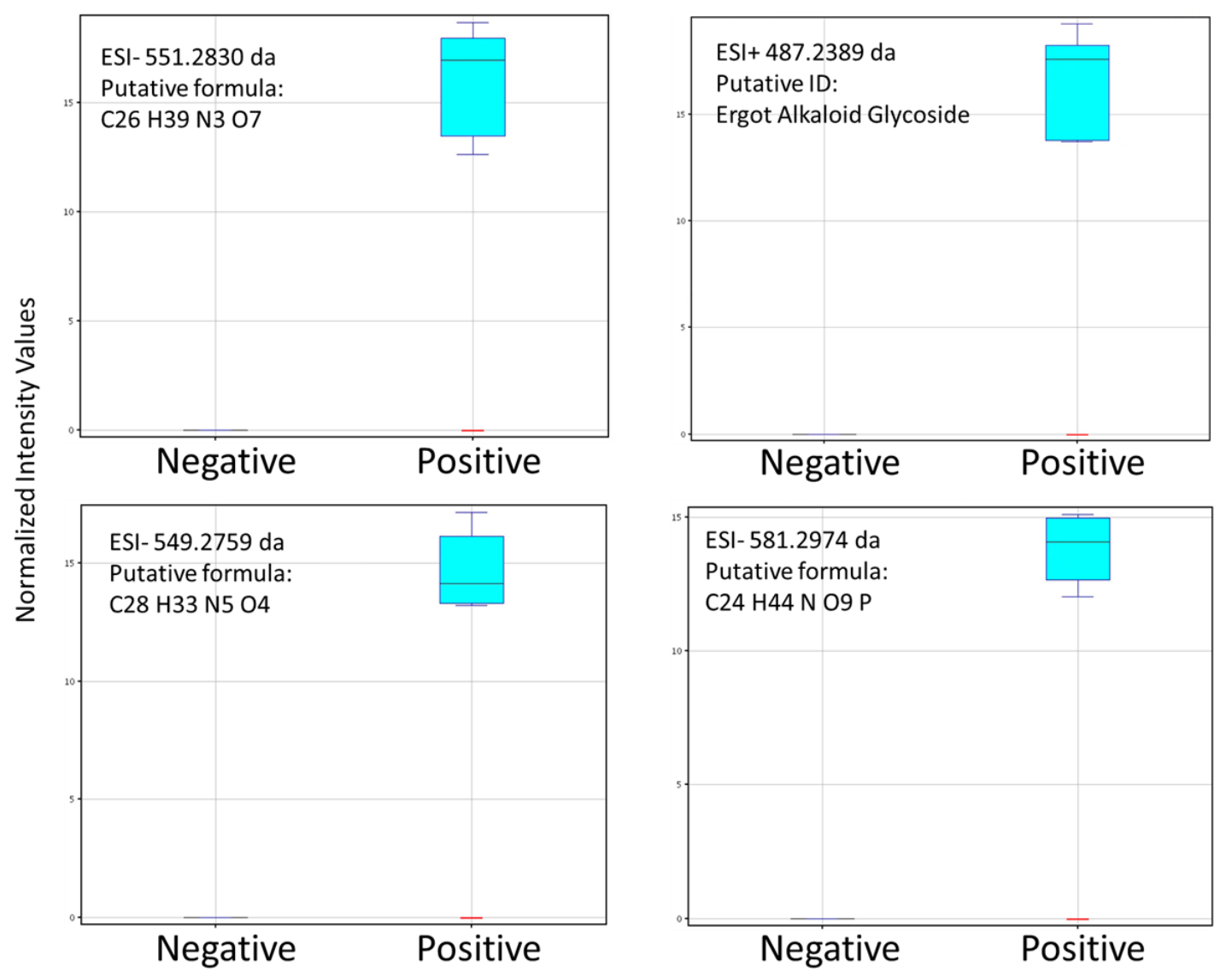

Figure 3 - Abundance profiles of four different metabolites from Ipomoea sp. grouped in ergot positive and ergot negative cohorts. 


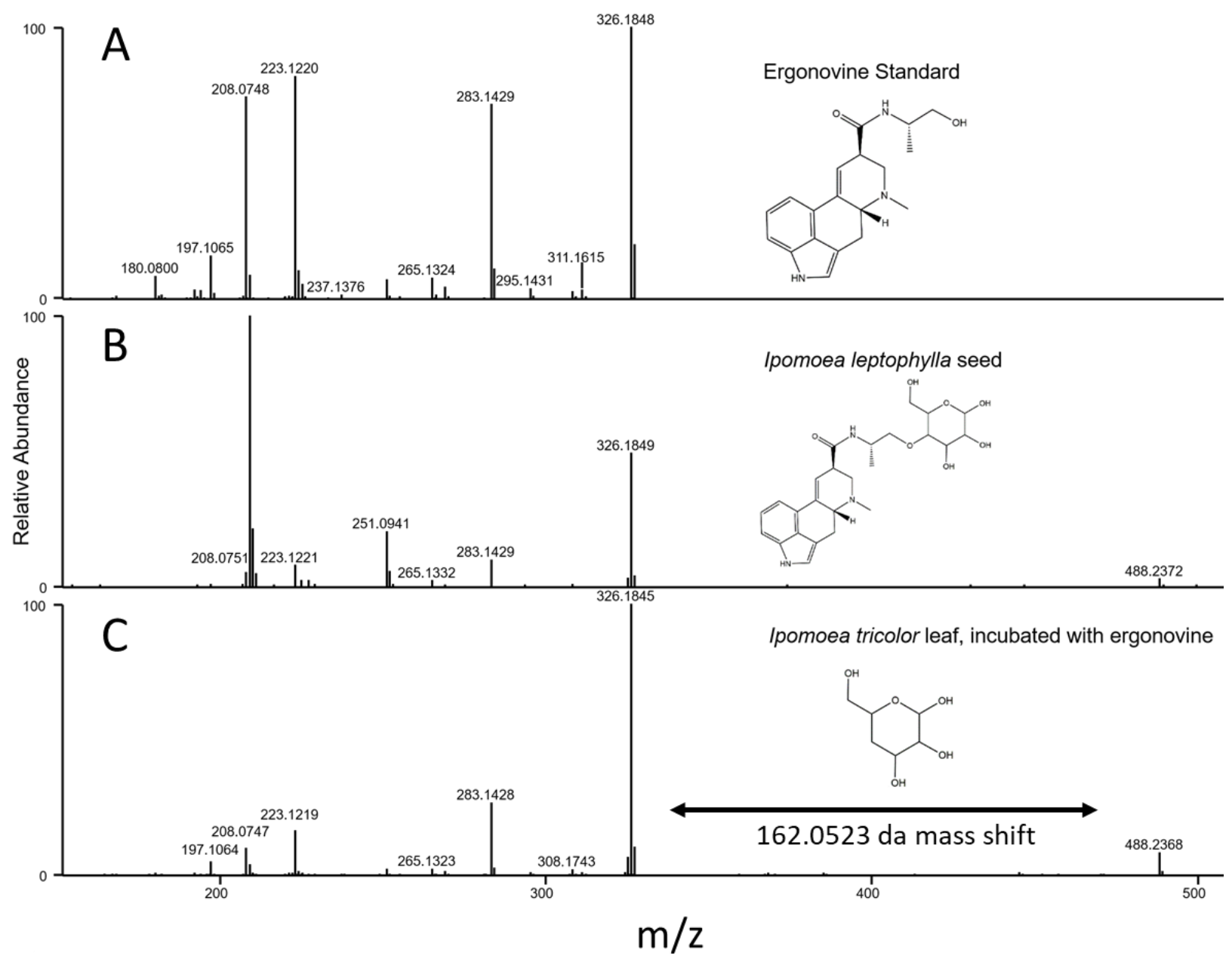

Figure 4. Q Exactive MS/MS spectra (RT $9.6 \mathrm{~min}$ ) from ergonovine (A), an ergonovine-like precursor from an I. leptophylla seed (B) and I. tricolor leaf incubated in an ergonovine solution (C). The mass shift seen in $B$ and $C$, from $488.237 \mathrm{~m} / \mathrm{z}$ to $326.185 \mathrm{~m} / \mathrm{z}$, is consistent with the mass of a hexose residue (-3.1 ppm). 


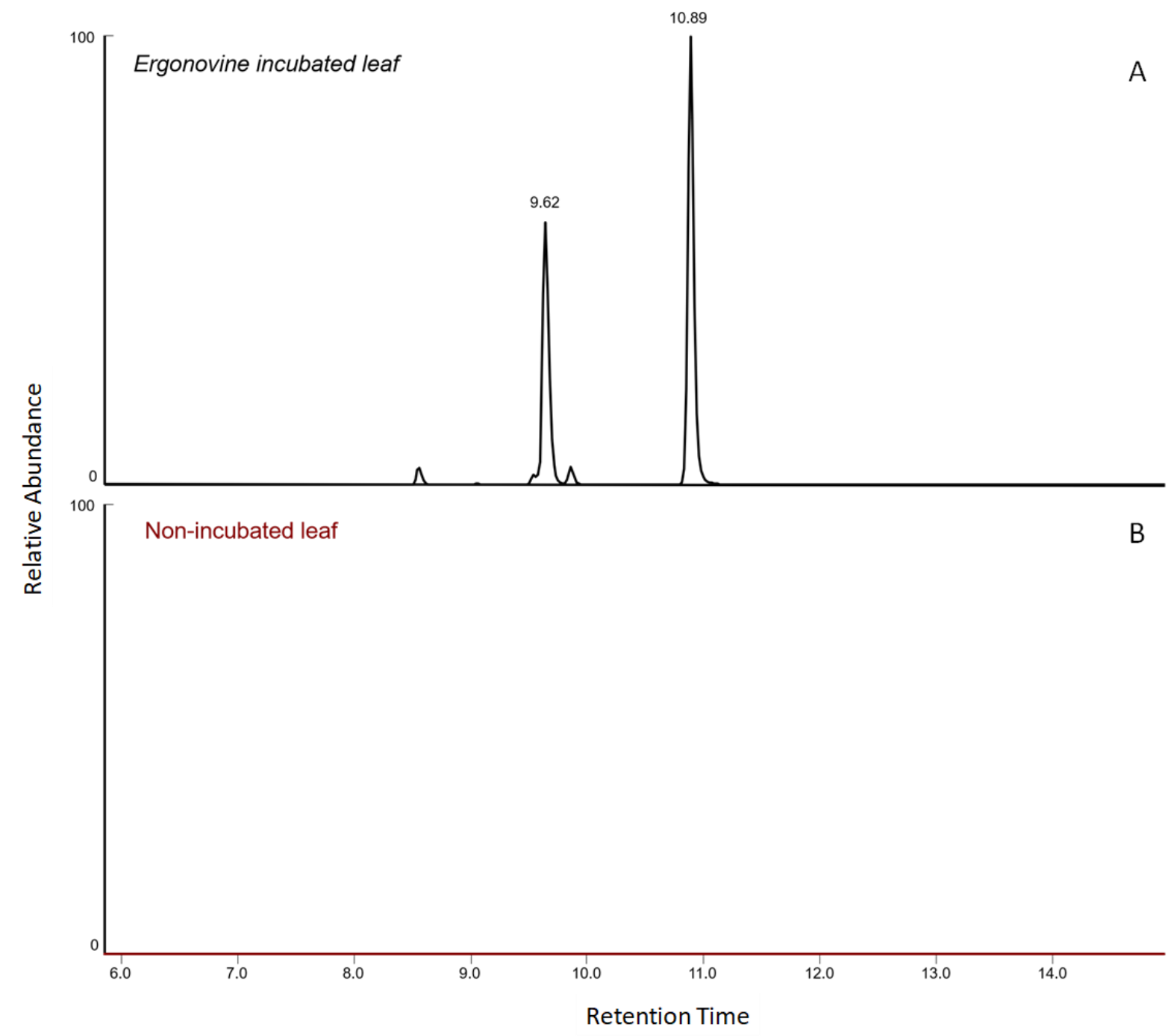

Figure 5. Extracted ion chromatogram $(488.239 \mathrm{~m} / \mathrm{z})$ generated on a $Q$ Exactive, from an ergonovine incubated $I$. tricolor leaf (A), and a non-incubated leaf (B). 


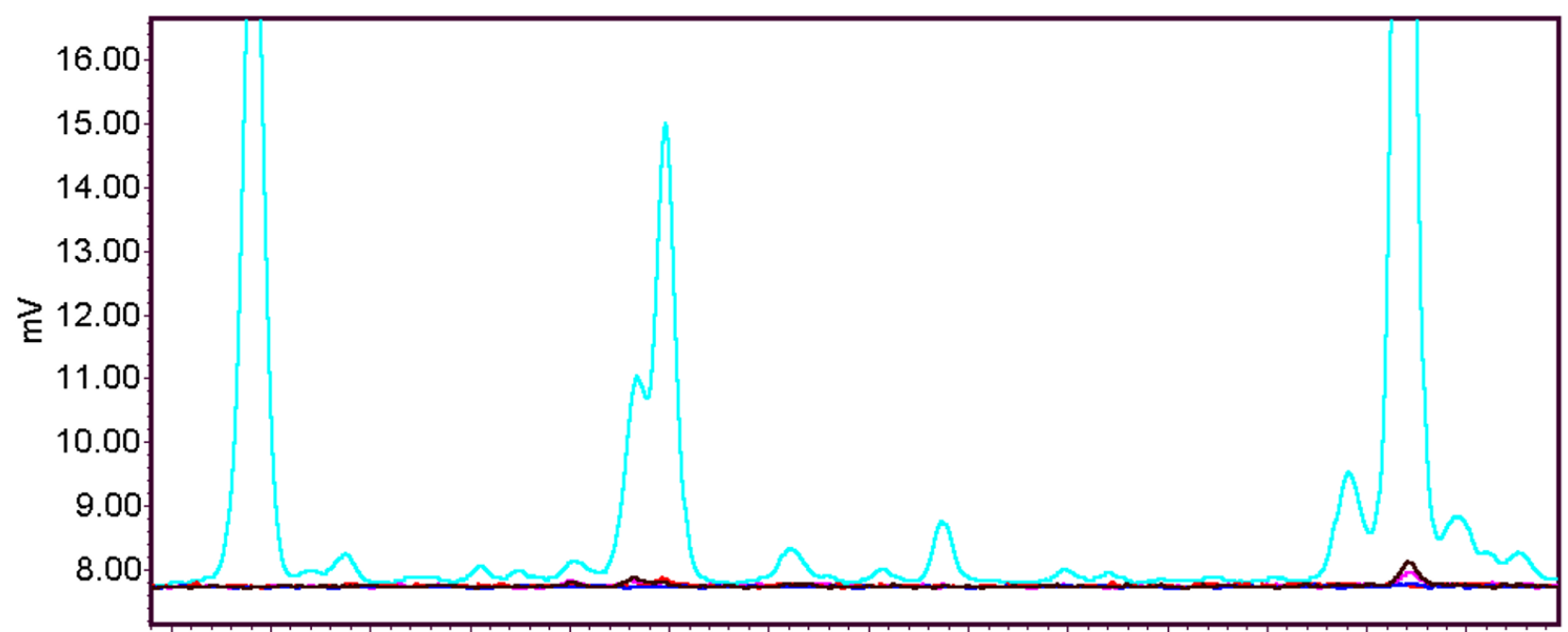

17.0018 .0019 .0020 .0021 .0022 .0023 .0024 .0025 .0026 .0027 .0028 .0029 .0030 .00 Minutes

Figure 6. Fluorescence chromatography of ergot alkaloids extracted from morning glory leaves. The ergonovine glycoside retains at $\mathbf{2 9 . 5}$ minutes with this method. The teal trace shows an intact leaf which was incubated in an ergonovine solution. The black trace shows a P+ leaf which was homogenized in a phosphate buffered saline solution containing ergonovine. The magenta trace shows a P- leaf which was homogenized in phosphate buffered saline containing ergonovine. The cyan and red traces show P+/Pleaves homogenized in PBS. 


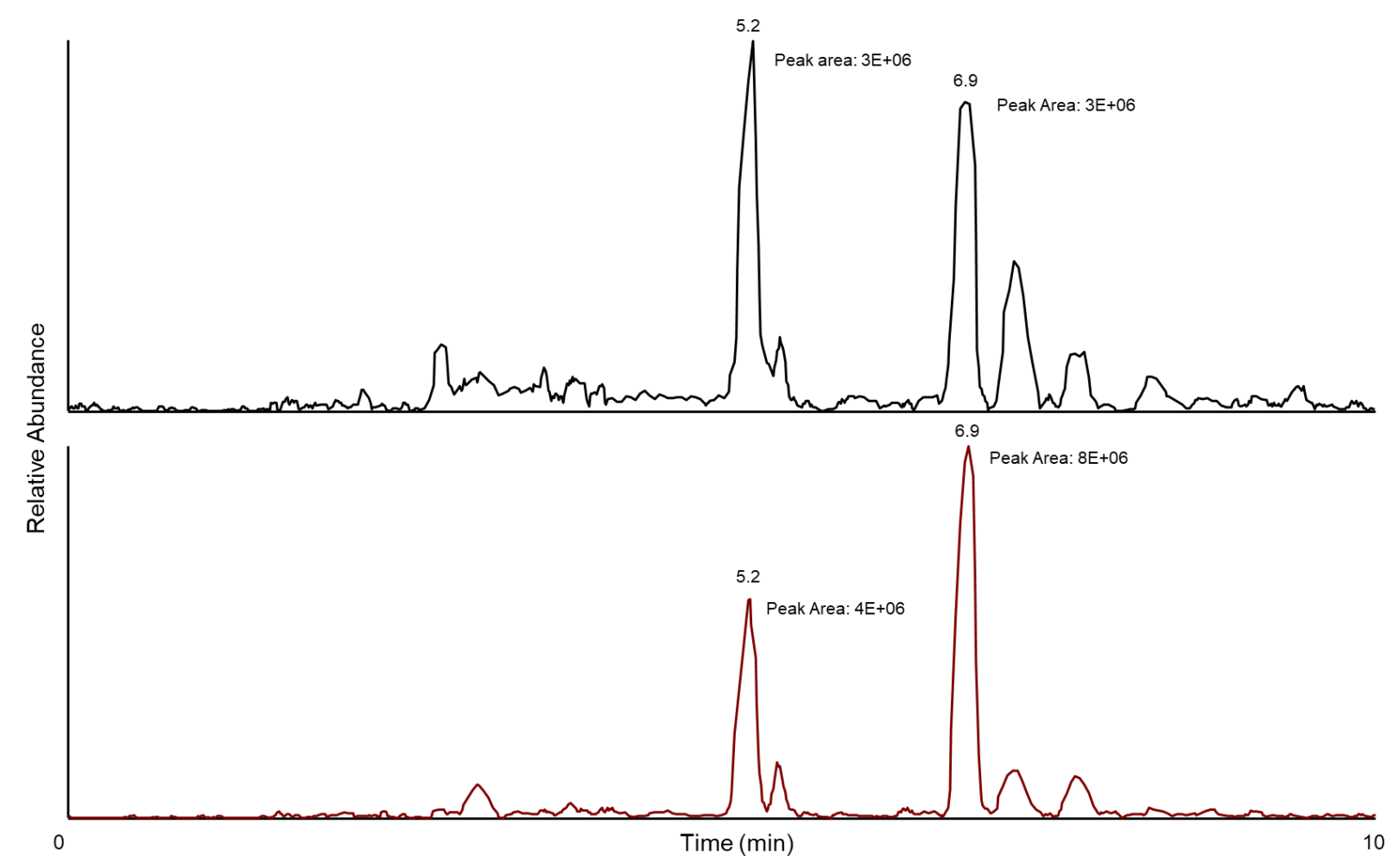

Figure 7a. Extracted ion chromatograms of $488.5 \mathrm{~m} / \mathrm{z}$, generated on a LCQDecaXP, from enzymatic synthesis of ergonovine glycoside. (A) UDP-glucose incubated with $\beta$-galactosidase, (B) UDP-galactose incubated with $\beta$-galactosidase. The peak area at 6.9 minutes grew a half order of magnitude in the sample incubated with UDP-galactose. 


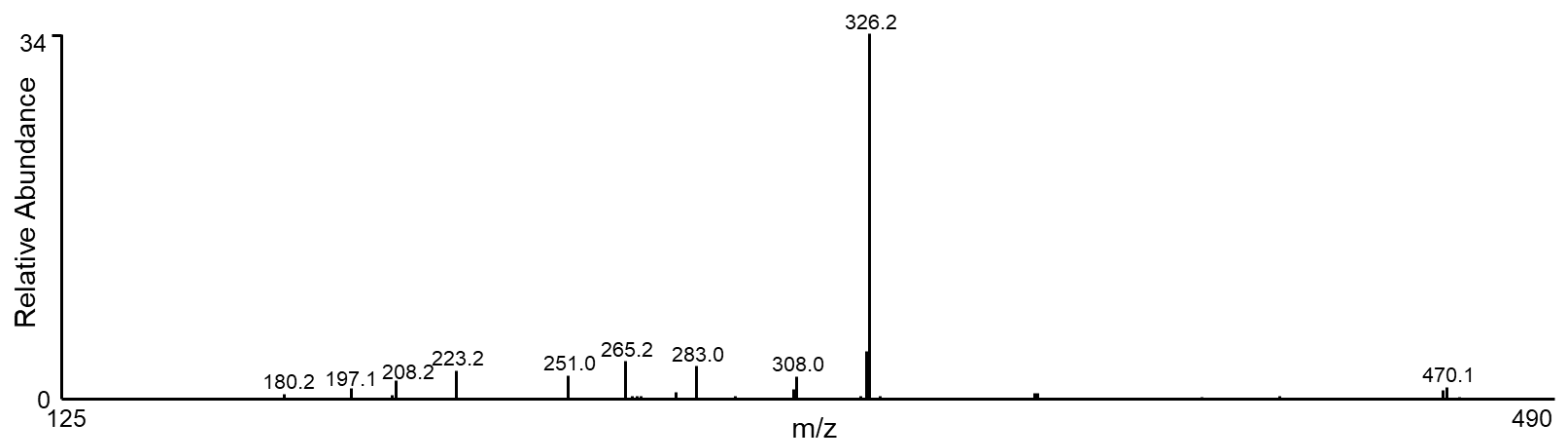

Figure 7b. Representative CID fragmentation spectrum, from a LCQDecaXP, of ergonovine glycoside. Due to the lower energy of the fragmentation technique, most of the abundance of the spectrum is represented by the $326.2 \mathrm{~m} / \mathrm{z}$ ergonovine fragment. The spectra is zoomed to show the lower mass peaks, matching those ions found in the HCD fragmentation spectrum. 

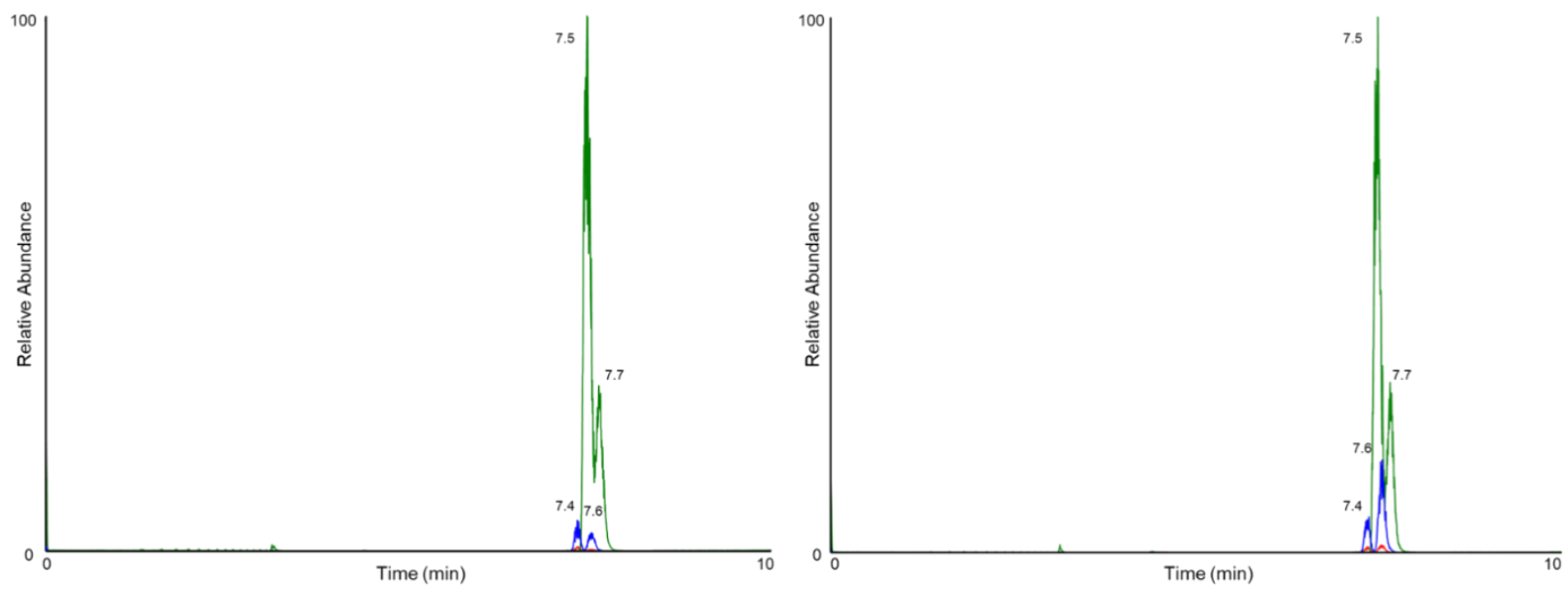

Figure 8. Rresults from a targeted LC-MS assay, generated on a 5500 QTrap, which monitors ergonovine (green trace) and the glycoside of ergonovine (blue). The trace on the left shows the result of incubating I. tricolor leaves with UDP-glucose and ergonovine, while the trace on the right shows I. tricolor leaves incubated with UDP-galactose and ergonovine. While the peak at 7.4 minutes remains at the same level between the two experiments, the peak at 7.6 minutes grows when UDP-galactose is added. 


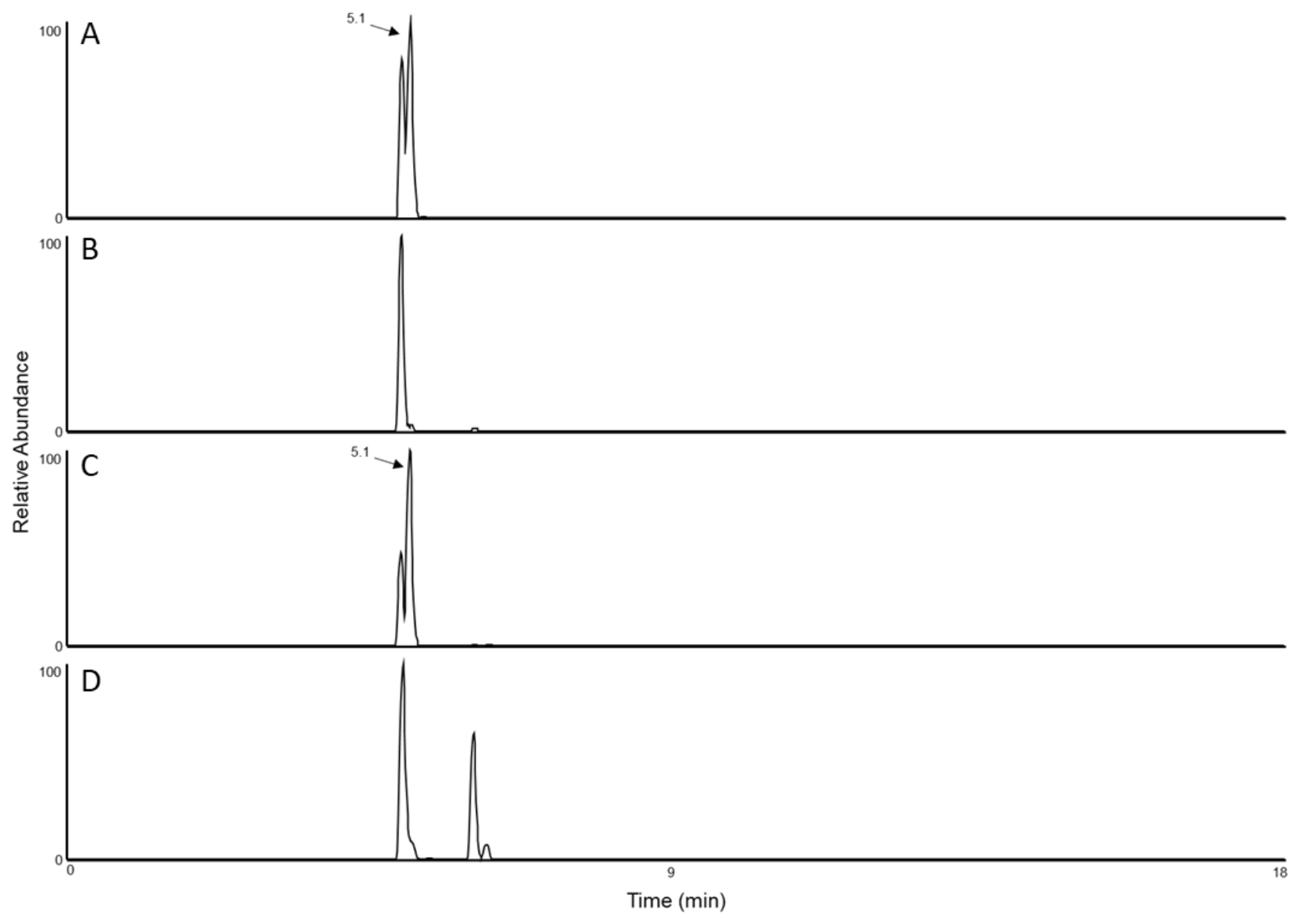

Figure 9. Extracted ion chromatograms showing $488.2931 \mathrm{~m} / \mathrm{z}$, from LC-MS analysis, on a Q Exactive, of four different $\beta$-galactosidase incubations. A peak matching the previously observed fragmentation spectrum for ergonovine glycoside was observed at 5.1 minutes. (A) $\beta$-galactosidase was incubated along with PNPGal and ergonovine at $\mathrm{pH} 4.5$ (B) $\beta$-galactosidase was incubated along with PNPGal and ergonovine at $\mathrm{pH} 4.5$, the solution was heated at $90^{\circ} \mathrm{C}$ for five minutes prior to incubation. (C) $\beta$ galactosidase was incubated along with PNPGal and ergonovine at $\mathrm{pH}$ 6. (D) $\beta$-galactosidase was incubated along with PNPGal and ergonovine at $\mathrm{pH} 6$, the solution was heated at $90^{\circ} \mathrm{C}$ for five minutes prior to incubation. 

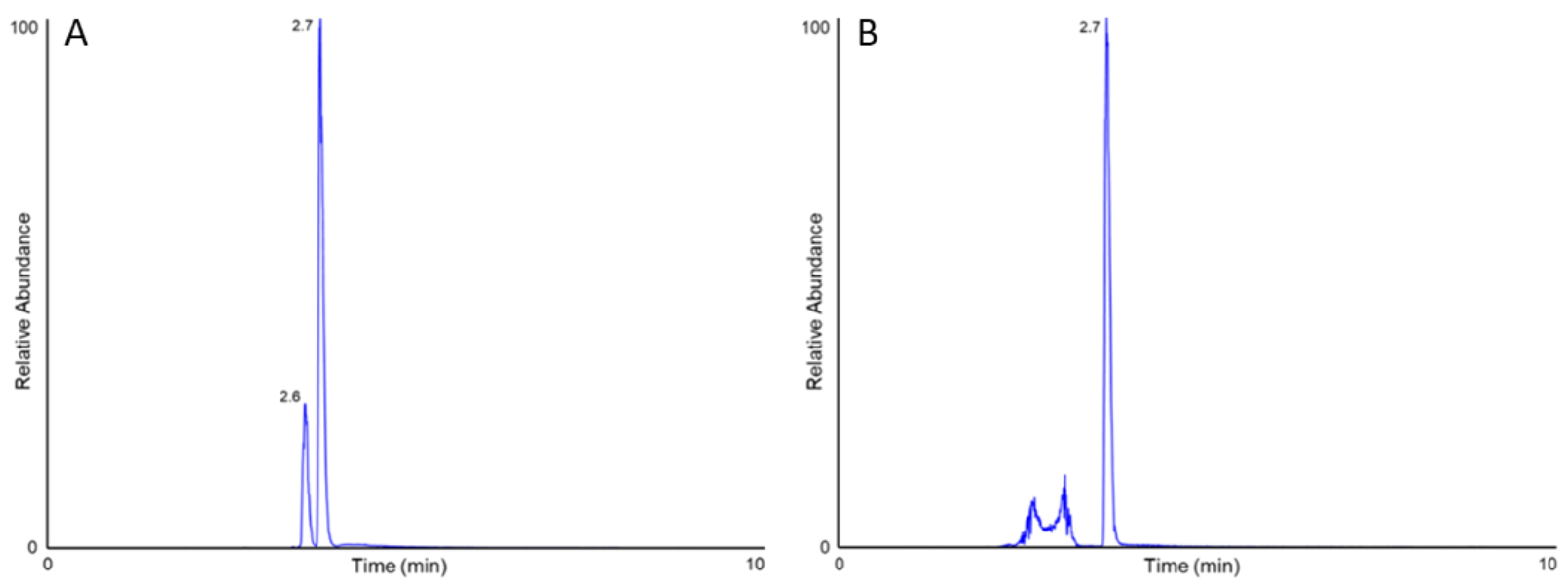

Figure 10. (A) Achnatherum inebrians, (B) Achnatherum robustum; Targeted LC-MS analysis ( $488 \mathrm{~m} / \mathrm{z} \rightarrow$ $326 \mathrm{~m} / \mathrm{z}$ ) using a 5500 QTrap, of ergot alkaloid extracts from two plants harboring ergonovine producing Epichlöe sp. A peak corresponding to ergonovine galactoside $(2.7 \mathrm{~min})$ can be seen in both extracts, $A$. inebriens may contain an earlier eluting alternate glycoform (2.6 min). 
Table 1. Profiling of ergot alkaloids

in Morning glory seeds by reversed phase fluorescence HPLC

\begin{tabular}{lc} 
Ipomoea species & Ergot alkaloids \\
\hline I. acantha & - \\
I. aquatica & - \\
I. barbatisepala & + \\
I. carnea & - \\
I. clavate & - \\
I. imperati & + \\
I. leptophylla & - \\
I. pandurata & $-/+$ \\
I. parasitica & a \\
I. pes-caprae & a \\
I. polpha & $-/+$ \\
I. tricolor $(\mathrm{P}+)^{\mathrm{b}}$ & - \\
I. tricolor $(\mathrm{P}-)^{\mathrm{c}}$ & + \\
I. tuboides & - \\
\hline
\end{tabular}

${ }^{a}$ of the seeds profiled, certain individuals contained or lacked ergot alkaloids

${ }^{\mathrm{b}}$ wild type seeds

${ }^{c}$ seeds from a lineage of plants that were cured of

Periglandula

Table 1. Seeds of various species of genus Ipomoea were tested by fluorescence HPLC for retention times matching known ergot alkaloids. Three species had individuals that contained ergot alkaloids and others that lacked ergot alkaloids. Those from I. tricolor that lacked ergot alkaloids were from a line of plants that had been cured of Periglandula ipomoeae infection. 


\section{Table 2. Species tested for ergonovine conversion to glycoside}

\begin{tabular}{lcc}
\hline \multicolumn{1}{c}{ Species } & Common name & Clade \\
\hline Achillea milleform & Yarrow & Asteridae, Asterales \\
Solanum lycopersicum & Tomato & Asteridae, Solanales \\
Ipomoea tricolor & Morning glory & Asteridae, Solanales \\
Tradescantia virginiana & Spiderwort & Commelinidae \\
Secale cerleale & Rye & Commelinidae \\
Zea mays & Corn & Commelinidae \\
Tilia cordata & European linden & Dilleniidae \\
Ginko biloba & Ginko & Ginkoopsida \\
Castaneamollissima & Chinese chestnut & Hamameliddae \\
Hemerocallis sp. & Day lily & Liliidae \\
Iris croatica & Bearded iris & Liliidae \\
Magnoliavirginiana & Sweetbay magnolia & Magnoliidae \\
Equisetum arvense & Horsetail & Pteridophyta \\
Cannabis sativa & Industrial hemp & Rosidae, Rosales \\
\hline
\end{tabular}




\section{CHAPTER 3 \\ THE IMPACT OF ERGOT ALKALOIDS ON METARHIZIUM BRUNNEUM SYMBIOSIS WITH INSECTS AND PLANTS}

\section{Abstract}

Ergot alkaloids are a group of specialized metabolites expressed by several fungi including species from the Clavicipitaceae. As members of the family Clavicipitaceae, several species belonging to the fungal genus Metarhizium have recently been shown to express lysergic acid derived compounds. Metarhizium species are prolific entomopathogens and have the capacity to form beneficial relationships with plants by colonizing their roots. A major goal of this study was to investigate the impact ergot alkaloid expression by Metarhizium brunneum had on the immune response of an insect host. A second goal was to determine how plant metabolite expression changes as a response to fungal symbiosis. Galleria mellonella larvae were injected with conidia selected from M. brunneum ARSEF 9354 and a strain of $M$. brunneum which had an ergot alkaloid synthesis gene knocked out. Insects infected with the knockout strain showed an altered melanization response compared to the wild type and control. LC-MS proteomic analysis showed that wild type and knock out strain infected insects had different protein expression profiles compared to each other as well as the control. Of particular interest, several antimicrobial peptides and proteins were downregulated in the ergot alkaloid expressing wildtype fungus. Metabolomics analysis demonstrated that stachydrine and $\curlyvee$-aminobutyric acid (GABA), among other compounds, were upregulated in the roots. These metabolites are known to promote microbial symbiosis and to promote stress-responses in plants. The results presented here, help to support the 
concept of $M$. brunneum as a biocontrol agent. This is the first report which suggests a potential mechanism for ergot alkaloids as a virulence factor for insect infection.

Some of the results presented in this chapter have been published as part of Leadmon et al (2020). In this chapter, I have excerpted and expanded upon my original contributions to that published study. 


\section{$\underline{\text { Introduction }}$}

Ergot alkaloids are a group of specialized metabolites expressed by several fungi from the Clavicipitaceae family. Representative genera from this family include Claviceps spp., Epichloë spp., Balansia spp., and Periglandula spp. (Florea et al., 2017; Robinson \& Panaccione, 2015). These fungi produce ergot alkaloids derived from lysergic acid, which have played a role in the development of medicine. As pharmacological understanding of these compounds has improved over time, synthetic derivatives of lysergic acid have been used to mitigate symptoms related to dementia, hyperprolactinemia, Parkinson's disease and migraines (Baskys \& Hou, 2007; Donnet et al., 2016; PerezLloret \& Rascol, 2010; Peer C. Tfelt-Hansen, 2013). Furthermore, these fungi have been shown to grow in close association with plants, either as mutualistic symbionts or as parasites. This plays a significant role in agriculture since lysergic acid derived compounds have been shown to cause toxic pathologies in humans and livestock resulting from the consumption of contaminated grains and forage crops (Craig et al., 2015; Florea et al., 2017; Haarmann et al., 2009; Klotz, 2015). While several studies have shown that ergot alkaloids can deter herbivory from mammals and insects, more research is needed to fully understand the role of ergot alkaloids in these fungal-plant relationships (Kaur et al., 2018; Panaccione et al., 2006; Parish et al., 2003a; Parish et al., 2003b; Potter et al., 2008).

As members of the family Clavicipitaceae, several species belonging to the fungal genus Metarhizium have recently been shown to have the capacity to express lysergic acid derived compounds (Leadmon et al., 2020). The ergot alkaloid gene cluster found in M. brunneum is composed of the same genes required in the pathway for the synthesis of LAH in Periglandula ipomoeae (Beaulieu et al., 2015; Hu et al., 2014; Schardl et al., 2013). Like other members in this family, Metarhizium species have the capacity to form beneficial relationships with plants by colonizing their roots. Furthermore, these fungi are also prolific entomopathogens, owing this lifestyle to their suite of virulence factors including 
hydrolytic enzymes and destruxins (Boldo et al., 2009; Duan et al., 2009; St. Leger et al., 1996, 2011; B. Wang et al., 2012). Because of the interaction between these two lifestyles, humans have been able to harness these fungi as biocontrol agents for agricultural applications (Faria \& Wraight, 2007).

Several studies have been published which investigated the gene expression response of insects infected by a variety of fungi, including Metarhizium sp. In the case of Metarhizium sp. It is well established that a variety of excreted proteins and metabolites from this genus, especially destruxins, modulate the insect immune response during infection (Mc Namara et al., 2017). Panaccione and Arnold (2017) found that ergot alkaloids contributed to the ability of $N$. fumigata to infect waxworms (Galleria mellonella). Since ergot alkaloid expression was only recently shown, it is unknown what role ergot alkaloids play as a virulence factor for Metarhizium sp. during insect infection. The goal of the study presented here was to investigate the impact ergot alkaloid expression by Metarhizium brunneum had on the immune response of its insect hosts, whether ergot alkaloids were produced in plant associations and how plant metabolite expression changes as a response to fungal symbiosis.

\section{Methods}

\section{Culture conditions and ergot alkaloid extraction}

Petri dish cultures of M. brunneum (ARSEF 9354) were grown on sucrose yeast extract agar composed of $20 \mathrm{~g}$ of sucrose, $10 \mathrm{~g}$ yeast exact, $1 \mathrm{~g}$ magnesium sulfate heptahydrate, $2 \mathrm{~mL}$ of trace elemental solution (Hutner et al., 1950), and $15 \mathrm{~g}$ agar (totaling to $1 \mathrm{~L}$ with water) for 7 days at room temperature. 


\section{LC-MS analysis of ergot alkaloid extracts}

Ergot alkaloids were extracted from $50-\mathrm{mm}^{2}$ agar-cutouts placed in methanol. These extracts were agitated and then centrifuged to pellet debris; supernatants were concentrated using a vacuum concentrator. A $2.1 \times 100 \mathrm{~mm}, 3.5 \mu \mathrm{m}$ particle size Zorbax SB-C18 LC column (Agilent Technologies, Santa Clara, CA) held at $40^{\circ} \mathrm{C}$ was used for reversed phase separations of $5 \mu \mathrm{L}$ injections of ergot alkaloid extracts. The analytical gradient ramped from $95 \%$ solvent $A(0.1 \%$ formic acid $)$ and $5 \%$ solvent $B(0.1 \%$ formic acid, acetonitrile) to $50 \%$ solvent B over 15 minutes, which was generated using an Accela 1290 UHPLC instrument (Thermo Scientific, San Jose, CA) at a flow rate of $300 \mu \mathrm{L} / \mathrm{min}$. A top five data dependent positive polarity electrospray ionization experiment using an in-line Q Exactive hybrid quadrupole Orbitrap mass spectrometer (Thermo Scientific, San Jose, CA) was used to detect and identify ergot alkaloids. Precursor scans were performed at 70,000 resolution over a detection window of 200 to $600 \mathrm{~m} / \mathrm{z}$. The top five most abundant ions from the precursor scan were isolated by the quadrupole (2 Da isolation window) for higher-energy collisional dissociation (HCD) set at a normalized collision energy (NCE) of 30. Fragment ions were then scanned with a resolution setting of 35,000.

\section{Waxworm hemolymph protein extraction and digestion}

Waxworm (Galleria mellonella) larvae were inoculated with $20 \mu \mathrm{L}$ PBS modified to contain $0.01 \%$ tween 20 and $10 \mathrm{ug} / \mathrm{mL}$ rifampin, or $20 \mu \mathrm{L}$ of modified PBS containing 800,000 conidia of $M$. brunneum eas $O$ knockout or wild type and then incubated at room temperature for 24 hours in quintuplicate. The extraction was performed according to the procedure that was previously described in the melanization assay, and then diluted to $100 \mu$ with PBS. Proteins were precipitated by adding $20^{\circ} \mathrm{C}$ acetonitrile at a 6:1 ratio (vol acetonitrile: vol hemolymph+PBS). Samples were then centrifuged at 
15,000 for 15 minutes at $4^{\circ} \mathrm{C}$. The protein pellet was resuspended in $50 \mathrm{mM}$ Tris, $1 \mathrm{mM} \mathrm{CaCl} 2$ buffered at pH 8.0 (digestion buffer). Protein concentrations were determined using A280 measurement on a NanoDrop One UV-Vis spectrophotometer (ThermoFisher Scientific, Waltham, MA). Protein concentrations were then normalized using digestion buffer. Cysteine bonds were disrupted by incubating in $1 \mathrm{M}$ dithiothreitol at $37^{\circ} \mathrm{C}$ for 60 minutes and were subsequently alkylated with $2 \mathrm{M}$ iodoacetamide in the dark at room temperature for 30 minutes. Trypsin was added at a 1:50 ratio (enzyme to substrate) and incubated at $37^{\circ} \mathrm{C}$ for 8 hours, and then acidified with $5 \mu$ of $1 \mathrm{~N} \mathrm{HCl}$.

\section{LC-MS sequencing and quantification of Galleria mellonella hemolymph proteins following}

\section{Metarhizium infection}

Peptides were introduced to the Q Exactive hybrid quadrupole Orbitrap mass spectrometer using a reversed phase gradient separation from an Acquity BEH C18, 130Å, $1.7 \mu \mathrm{m}, 1 \mathrm{~mm}$ X $150 \mathrm{~mm}$ UPLC column (Waters Corporation, Milford, MA) on an Accela 1290 UHPLC instrument. The column was equilibrated with $98 \%$ mobile phase A $(0.1 \%$ formic acid, water) and then a gradient ramped from $2 \%$ mobile phase B ( $0.1 \%$ formic acid, acetonitrile) to $38 \%$ B over 54 minutes at a flow rate of $150 \mu \mathrm{L} / \mathrm{min}$ held at $45^{\circ} \mathrm{C}$. The mass spectrometer was operated in positive ionization mode with a data dependent acquisition scheme. Precursor ions were monitored between $300-1750 \mathrm{~m} / \mathrm{z}$ at 70,000 resolution. The top 10 most abundant multiply charged ions (charge state 2-6) were selected for sequencing by MS/MS with a 20 second dynamic exclusion list. Precursor ions were selected using a $2.0 \mathrm{~m} / \mathrm{z}$ isolation width and were fragmented by and HCD setting of 28 NCE. Fragment ions were scanned at 17,500 resolution.

Peptide searches and relative quantification were performed using Proteome Discoverer 2.4 (Thermo Scientific, Waltham, MA) and the Sequest HT search engine against the Uniprot Galleria mellonella TrEMBL database. The search was configured in the following manner: required fully tryptic 
sequence; up to two missed cleavages; fixed modification of carbamidomethylation (C) and dynamic modifications of oxidation (M) and $\mathrm{N}$-terminal acetylation; precursor mass tolerance set to $10 \mathrm{ppm}$ and a fragment mass tolerance of $0.02 \mathrm{Da}$. Protein and peptide results were filtered based on a minimum of two unique peptides and a 1\% FDR calculated from the number of hits in the search against a concatenated database of reverse protein sequences. Minora Feature Detector was used to align precursor ion peaks in a $10 \mathrm{ppm}$ window, linked to peptide spectral matches with a high confidence level $(q<0.01)$. The peak areas of the two most abundant peptides for each protein were averaged to generate an abundance value normalized against the total abundance of all peptides from each analytical run. Sample group protein abundance ratios were generated pairwise verses each treatment type and filtered based on a minimum Log2 fold change of one. Quantitative significance was assessed on the individual protein level by ANOVA $(p<0.05)$ with a Benjamini-Hochberg adjustment to compensate for multiple comparisons.

\section{Hemolymph melanin measurement}

Hemolymph was extracted from waxworm larvae inoculated with PBS, M. brunneum easO knockout or wildtype and then incubated at room temperature for 6 hours in triplicate. The extraction was performed according to the procedure that was previously described the proteomic study. Hemolymph was centrifuged at $10,000 \mathrm{rpm}$ for 10 minutes at $4^{\circ} \mathrm{C}$ to remove cellular debris and then transferred to 96 well flat bottom plate for analysis. Melanization was measured by absorbance at 470 $\mathrm{nm}$ in a Synergy HTX plate reader (BioTek, Winooski, VT). Differences in absorbance were assessed using JMP (SAS, Cary, NC). A Brown-Forsythe test was used to check for unequal variances, while means were assessed with ANOVA and a post-hoc Tukey's Test. 


\section{LC-MS metabolomics of Zea mays inoculated with Metarhizium sp.}

Plants used in the following studies were prepared according to the procedures described in Leadmon et. al (2020). Polar metabolites were separated using HILIC chromatography, by injecting $10 \mu \mathrm{L}$ of extracts onto a $2.1 \times 100 \mathrm{~mm}, 5 \mu \mathrm{m}$ Phenomenex Luna NH2 column (Torrance, CA). A 15 minute linear gradient, which ramped from $90 \%$ solvent B (100\% acetonitrile) to $60 \%$ solvent $A$ (10 mM ammonium acetate) at a flow rate of $300 \mu \mathrm{L} / \mathrm{min}$, was used to elute the metabolites for detection by electrospray ionization mass spectrometry. Each sample was analyzed in positive and negative ionization modes. The LC-MS system used for analysis consisted of an Infinity 1290 ultra-high pressure liquid chromatography instrument (Agilent Technologies, Santa Clara, CA) coupled to an Agilent 6530 quadrupole time of flight (QToF) mass spectrometer configured with a jet stream source for electrospray ionization. For both positive and negative ionization modes, the mass spectrometer scanned over a range from 50 to 1200 $\mathrm{m} / \mathrm{z}$ with a scan speed of $2 \mathrm{hz}$.

\section{Analysis of Metabolomics Data}

LC-MS data were processed using Agilent Masshunter Profinder (version B.06.00) in batch mode according to polarity of acquisition. Chromatographic features were extracted which contained a minimum peak height of 600 counts and a maximum charge state of 2 . The resulting chromatography was inspected for consistency between samples, with retention time and mass alignments performed on irregular features. Peak areas from these chromatographic features were exported to Mass Profiler Professional (version 2.4.3) for further filtering, quality control and statistical analysis. Differences in peak abundances of chromatographic features between treatment types were assigned significance by a Bonferonni adjusted T-test $(p<0.05)$, with a minimum fold change $>5$. 


\section{Compound Identity confirmation by tandem MS analysis}

Those compounds identified as significantly changing in the previous step were re-analyzed using MS/MS fragmentation. Metabolite extracts were pooled according to sample type, and $20 \mu \mathrm{L}$ of metabolite extract were injected onto an Accela 1290 UHPLC instrument configured for HILIC chromatography (according to the previously described separation), coupled to a Q Exactive hybrid quadrupole Orbitrap mass spectrometer. Injections were repeated for analysis in positive and negative ionization modes. The instrument was operated in a data dependent acquisition mode with an inclusion list generated from the significant compounds in the QToF analysis, a match tolerance of $10 \mathrm{ppm}$ was used. A precursor scan of 80 to $1200 \mathrm{~m} / \mathrm{z}$ was generated using the 70,000 -resolution setting. The top ten most abundant singly charged ions from the precursor scan were isolated by the quadrupole (2 Da isolation window) for HCD fragmentation at an NCE of 30. Fragment ions were then scanned with a resolution setting of 35,000. The resulting data were searched using Compound Discoverer 2.1 (Thermo Scientific, Waltham, MA) against the KEGGS, BioCyc and Metlin metabolite libraries using a mass match tolerance of $10 \mathrm{ppm}$.

\section{$\underline{\text { Results }}$}

\section{Confirmation and identification of ergot alkaloids expressed by Metarhizium brunneum}

High-resolution mass spectrometry data confirm the accumulation of chanoclavine, lysergic acid a-hydroxyethylamide (LAH), ergonovine, and ergine (spontaneously formed from LAH) in $M$. brunneum cultures (figure 1a), consistent with HPLC and unit mass resolution LC-MS data (Leadmon et al., 2020). Also observed for the first time was ergotryptamine, an early pathway spur product synthesized through the activities of DmaW, EasF, and EasC (Ryan et al., 2013, 2015). All compounds were observed within a narrow 10 ppm mass window, and fragmentation patterns match the expected patterns for all compounds (figure $1 b$ ). 


\section{LAH synthesizing $M$. brunneum modulates the melanization response in waxworms}

To test the effect of LAH as a virulence factor for M. brunneum, waxworms were injected with conidia selected from M. brunneum ARSEF 9354 and a hypervirulent strain of M. brunneum which had easO knocked out, the gene for the committed biosynthetic step for LAH production. In addition to its lack of ergot alkaloids, the eas $O$ mutant kills insects more rapidly than the wild type but does not emerge from the corpse of the insect (Tyo and Panaccione, 2018). In these ways the eas $O$ mutant provides a stark contrast to the wild type strain. Hemolymph was extracted six hours after injection and tested for the melanization response by measuring absorbance at $470 \mathrm{~nm}$. The insects that were injected with the eas $O$ knockout strain of $M$. brunneum demonstrated a significantly lower level of melanization as compared to the PBS control and the wild type fungus $(P<0.001$, ANOVA) (figure 2$)$. Insects were screened by HPLC analysis to confirm that absence of LAH in easO knockout infected insects. These data suggest that ergot alkaloids play a role in the regulation of insect melanization as a response to fungal infection.

\section{Waxworm antimicrobial proteome expression is suppressed by LAH synthesizing $M$. brunneum}

Hemolymph was recovered from G. mellonella larvae for assessment of differences in protein expression between the PBS control and the two types of $M$. brunneum infections (eas $O$ knockout and wild type). Analysis of these proteomes yielded unique protein expression profiles based on treatment type. PCA analysis generated from protein abundance profiles $(P<0.05)$ showed that samples from each treatment type group together within their respective cohorts but separately from the other treatment types (figure 3). Furthermore, when comparing normalized abundance profiles of significantly changing proteins $(P<0.05)$ using Euclidean farthest neighbor hierarchical clustering, the wildtype infections, and the PBS control treatments cluster together and apart from easO knockout strain infections (figure 4). 
Volcano plots, contrasting log2 fold change with significance were generated to identify individual proteins which had their expression significantly changed by the treatment types (figure 5). A total of 148 proteins were quantified among the three groups. Of the proteins monitored, this present study provides evidence at the protein level for the expression of 44 genes, which were previously only observed by mRNA sequencing. A total of 21 proteins were found to be differentially expressed in at least one of the three treatment groups (table 1), with 6 of these proteins being differentially expressed between the eas $O$ knockout infected larvae and the wild type-infected larvae. These expression profiles of the wild type-infected larvae showed a decrease in abundance for G. mellonella orthologs of four (or is it three?) antimicrobial peptides (figure 6) - gloverin (-3.1 $\log _{2}$ fold change), moricin (-1.2 $\log _{2}$ fold change), proline rich antimicrobial peptide (-2.7 $\log _{2}$ fold change) and peptidoglycan recognition protein $\left(-0.8 \log _{2}\right.$ fold change).

\section{M. brunneum colonization alters the metabolome of Zea mays}

Since plant roots are also an ecological niche occupied by Metarhizium species, I analyzed how colonization by Metarhizium species affected the metabolome of corn (Zea mays). When the roots of filter-paper sprouted corn seedlings were exposed to inocula from M. brunneum and M. flavoviride, fungal mycelia were observed growing on the surface of the plants. Since homogenates of these plants were negative for ergot alkaloids when tested using an FLD-HPLC assay (Leadmon et al., 2020), metabolomics analyses also provided a means for demonstrating colonization of the plants by the fungi. Global metabolite profiling was performed on homogenized plant tissues using HILIC-LC-MS analysis. Of the $>1000$ chromatographic features which were monitored (ergot alkaloids also were not detected in these independent analyses), 66 had altered expression profiles between plants inoculated with $M$. brunneum, M. flavoviride, or non-inoculated control plants (table 2). The identities of these compounds 
and their relative abundance profiles can be found in table 3 . Of particular note, $\curlyvee$-aminobutyric acid was observed with a $20-\log _{10}$ fold higher abundance in roots infected with $M$. brunneum and a $18-\log _{10}$ fold higher abundance in roots infected with $M$. flavoviride $(P<0.05)$.

\section{Discussion}

The accurate mass results presented here confirm the ergot alkaloid profile produced by $M$. brunneum as previously reported (Leadmon et al., 2020). These ergot alkaloids were shown to accumulate in M. brunneum infected $G$. mellonella, with lysergic acid $\alpha$-hydroxyethylamide being the most abundant. A role for ergot alkaloids in modulating an insect's immune response to Metarhizium sp. has not been investigated, until now. The genetically modified ergot alkaloid deficient strain of $M$. brunneum proved to be a useful tool for probing these interactions. An important point to note regarding the eas $O$ mutant of $M$. brunneum used in the present study is that its chemotype is a lack of all ergot alkaloids. Based on genotype, an easO deficient strain of $M$. brunneum would be expected to accumulate ergot alkaloids up through and including ergonovine. The lack of all ergot alkaloids in the eas $\mathrm{O}$ mutant used here indicates further mutations or a change in gene regulation. Nonetheless, the strains lack of ergot alkaloids and hypervirulent phenotype provide an altered background against which the wild type of $M$. brunneum can be compared.

Melanization is a well characterized physiological pathway in insects, whereby they respond to a variety of stimuli, including infection (Nakhleh et al., 2017). The melanization assay performed here was used as a measure of the insect immune response. These data suggest that the regulation of insect immunity as a response to infection by a fungal pathogen is modified by ergot alkaloids and point to a role for these compounds as virulence factors. 
Global hemolymph proteome analysis was used to further investigate the role of ergot alkaloids in Metarhizium virulence. Principal component analysis (figure 3) of the observed changing proteins confirms what was seen in the melanization assay. The protein expression profiles cluster together according to infection type, with the wild-type infections separating away from the eas $O$ knockout infections, with the PBS control separating into its own distinct group. When this is taken into consideration with the hierarchical clustering analysis (figure 4), the proteomic differences indicate that while the M. brunneum infected waxworms do mount some response to the presence of the fungus. Indeed, several antimicrobial peptides and defense related proteins were measured and were expressed at similar levels between the two types of infections (table 1). However, the overall insect proteome in wild-type M. brunneum infections is more like what is found in a healthy individual than an individual that has been infected by the hypervirulent strain of $M$. brunneum lacking ergot alkaloids, with several immune related peptides and proteins being downregulated. This difference in immune response is not surprising, as different microorganisms have been shown to elicit differential immune peptide responses in G. mellonella from different pathogens (Mak et al., 2010).

The interesting part, that may point to a role for ergot alkaloids, is that six proteins were differentially regulated between Metarhizium brunneum infections with and without ergot alkaloids (figure 6). Among these proteins gloverin, moricin and the proline-rich peptide are known as antimicrobial peptides and have been shown to be involved in insect immune response (Mc Namara et al., 2017; Vogel et al., 2011). The Manduca sexta (tobacco hornworm) homolog of the gloverin-like protein, has been shown in to bind cellular components of a variety of microbes and present antimicrobial activity against bacteria and fungi alike (X. X. Xu et al., 2012). The proline-rich peptide identified here may be related to proline-rich peptides from other lepidopteran species, which are known to have antimicrobial activity against gram-negative and gram-positive bacteria, as well as fungi (Yi et al., 2014). A recent study where G. mellonella was challenged by Candida albicans infection 
showed elevated peptidoglycan recognition-like protein as a response (Sheehan \& Kavanagh, 2018). Moricins are a group of antimicrobial peptides originally identified in the lepidopteran insect Bombyx mori (Hara \& Yamakawa, 1995), more recently G. mellonella was shown to have its own unique repertoire of moricin-like peptides (Brown et al., 2008). In that study moricin A (also identified in this present study) was shown to be especially active against the filamentous fungi Fusarium graminearum, Fusarium oxysporum, Ascochyta rabiei, and Leptosphaeria maculans. All of this together suggests that the waxworm immune response is limited in scope when challenged by a fungus using ergot alkaloids as a virulence factor. Indeed, a similar conclusion was reached by Panaccione and Arnold (2017) for the distantly related fungus Neosartorya fumigata and its ergot alkaloids. This study suggests that the actual protein targets of ergot alkaloids are regulatory elements upstream of both the melanization response and the antimicrobial peptides identified as changing in this study. Further research could be performed to associate genes in the waxworm with this response.

While Metarhizium brunneum is effective as an entomopathogen, it also readily grows in association with plants. However, one major distinction can be made with regards to that association unlike with insects, $M$. brunneum does not produce ergot alkaloids when grown in conjunction with plants. Despite this, the metabolomic data presented here show that M. brunneum does not grow as a passive participant with its plant host, several metabolites were identified as changing when the plant was grown with the fungus present. Two of these metabolites, stachydrine and $\curlyvee$-aminobutyric acid (GABA) are particularly interesting. Stachydrine has been reported as being involved in promoting plant nodulation and microbial symbiosis, as it is released from the seeds of Medicago sp. and in turn activates nod genes in the symbiotic bacteria Sinorhizobium meliloti (Cooper, 2007). As for GABA, plants use this compound as a signaling molecule to regulate growth and development (Ramesh et al., 2017). Several reports have been released in recent years which indicate that supplementing a plant with GABA 
helps to promote responses to stress, including low-light conditions and high-salinity (Li et al., 2017; Vijayakumari \& Puthur, 2016; J. Xu et al., 2019).

The results presented here, help to support the concept of $M$. brunneum as a biocontrol agent. The ability of $M$. brunneum to grow in an association with a plant host, and promote certain key growth factors, while not producing ergot alkaloids make it suitable to be used with food crops. Furthermore, while it has already been shown that $M$. brunneum uses ergot alkaloids as a virulence factor in its role as an entomopathogen, this is the first report which suggests a potential mechanism. 


\section{Figures}

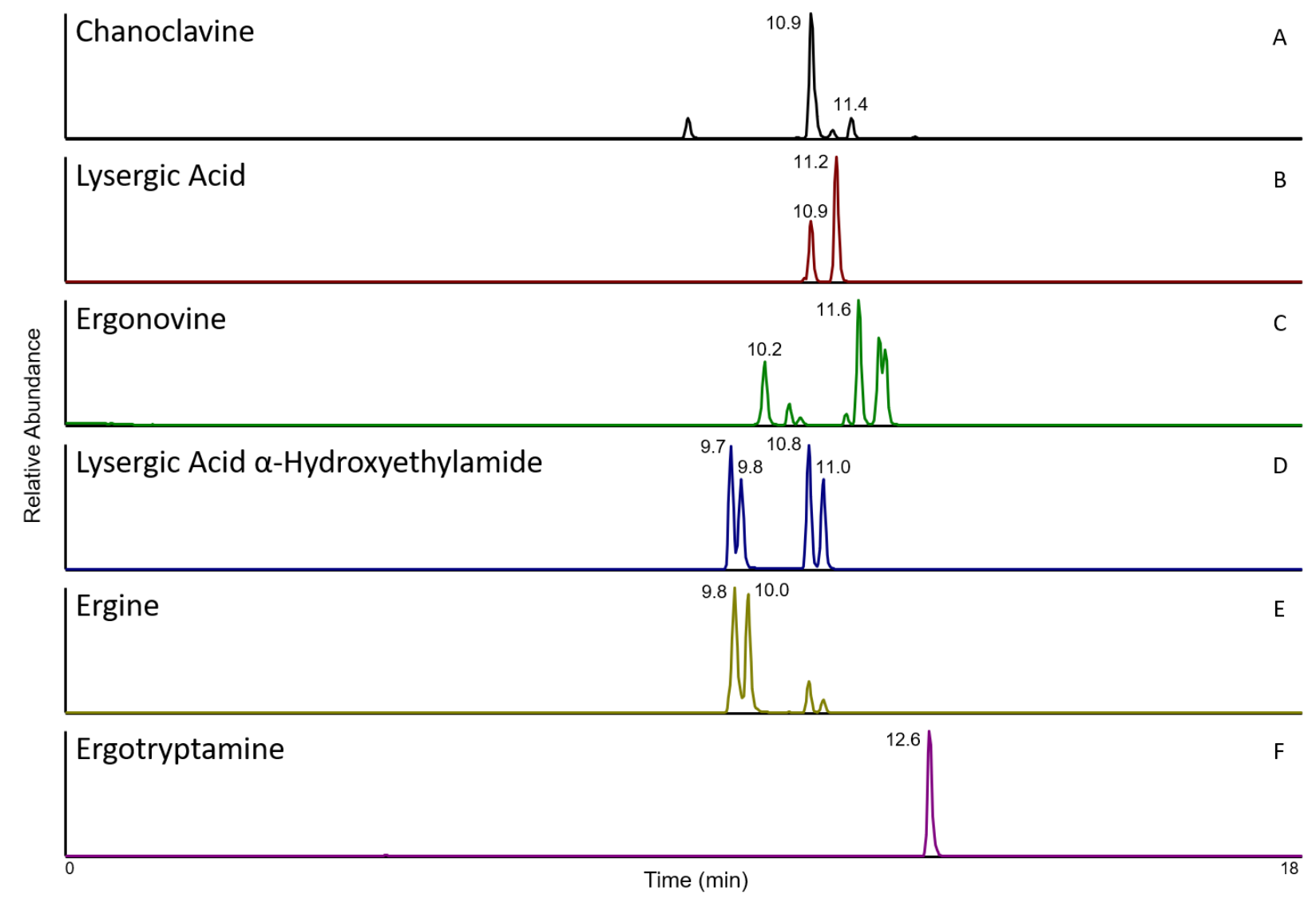

Figure 1a. Extracted ion chromatography, from a Q Exactive, of ergot alkaloids observed in M.

brunneum. (A) chanoclavine, $257.1649 \mathrm{~m} / \mathrm{z}$, (B) lysergic acid, $269.1285 \mathrm{~m} / \mathrm{z}$, (C) ergonovine, 326.1863

$\mathrm{m} / z,(\mathrm{D})$ D-lysergic acid $\alpha$-hydroxyethylamide (LAH), $312.1707 \mathrm{~m} / \mathrm{z}$, (E) ergine, $268.1445 \mathrm{~m} / \mathrm{z},(\mathrm{F})$

ergotryptamine, $259.1805 \mathrm{~m} / \mathrm{z}$. All ions were extracted with a $10 \mathrm{ppm}$ mass window centered on the theoretical monoisotopic $\mathrm{M}+\mathrm{H}$. The multiple peaks observed in most compounds are chromatographically resolved stereoisomers. Tandem MS spectra were used to assign identities for the labeled retention times. 


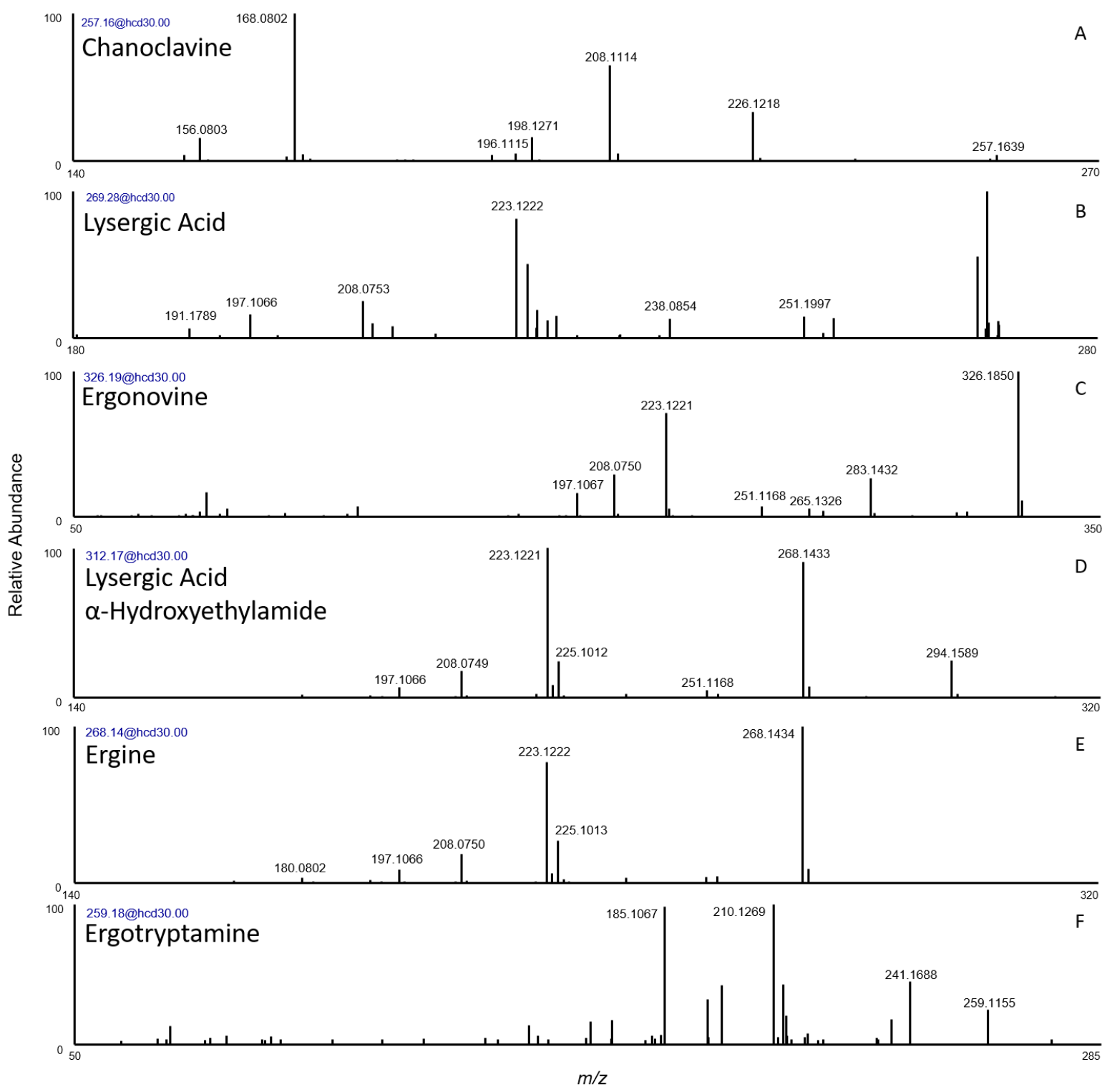

Figure $\mathbf{1 b}$. High resolution, accurate mass HCD fragmentation spectra, for ergot alkaloids detected in $M$. brunneum. (A) chanoclavine, (B) lysergic acid, (C) ergonovine, (D) D-lysergic acid $\alpha$-hydroxyethylamide (LAH), (E) ergine, (F) ergotryptamine. Later pathway intermediates (B-E) show common fragment ions $197.107 \mathrm{~m} / \mathrm{z}, 208.075 \mathrm{~m} / \mathrm{z}$, and $223.122 \mathrm{~m} / \mathrm{z}$. 


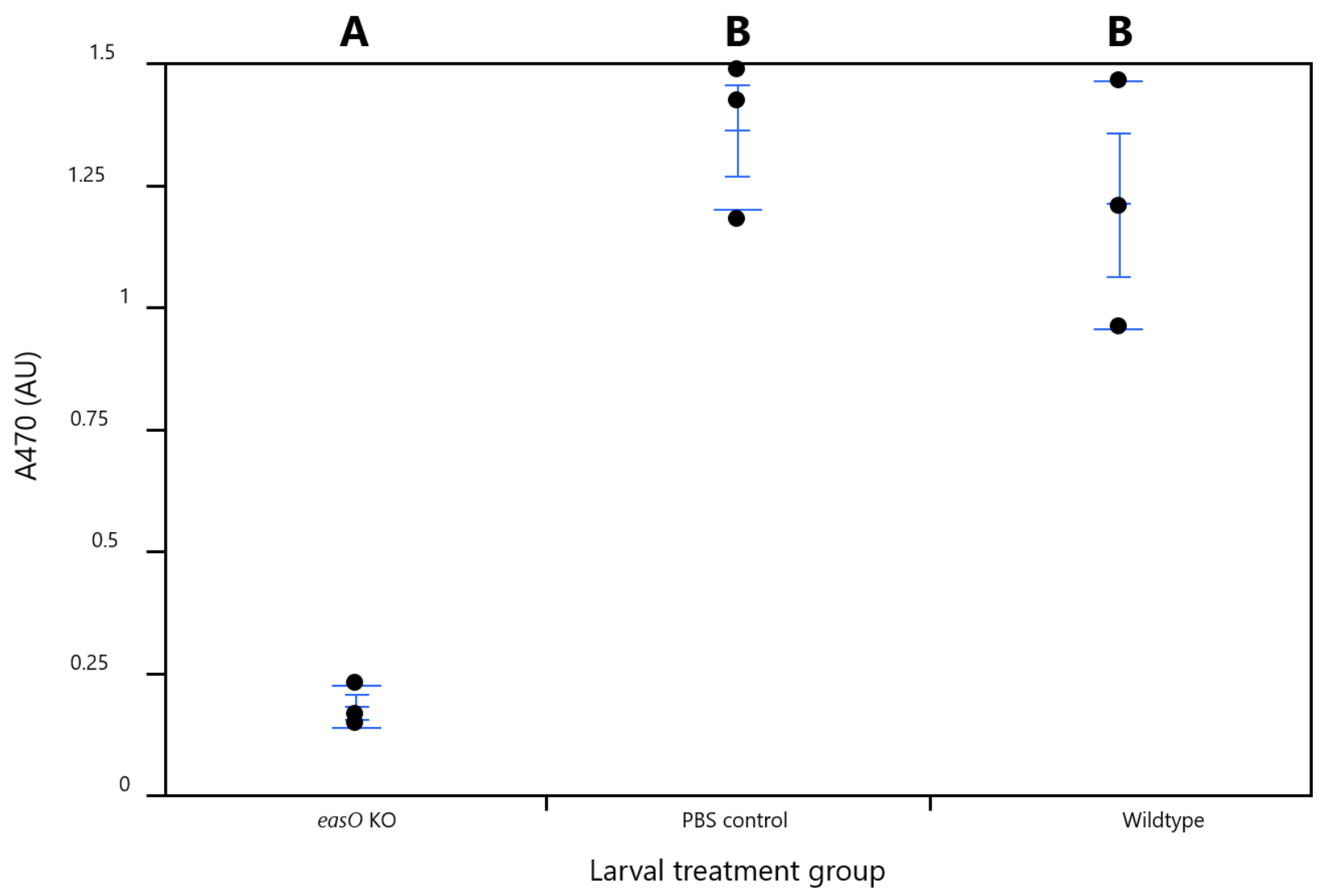

Figure 2. Abundance DOPA (measured by absorbance at $470 \mathrm{~nm}$ ) as a proxy for melanization responses for waxworm larvae injected with easO knockout M. brunneum, wild-type M. brunneum, and sterile phosphate buffered saline control. A comparison of all pairs using Tukey-Kramer HSD, showed that mean abundance response for the PBS control and wild-type fungus were similar (B), and both differed from the response for the easO knockout (A). 


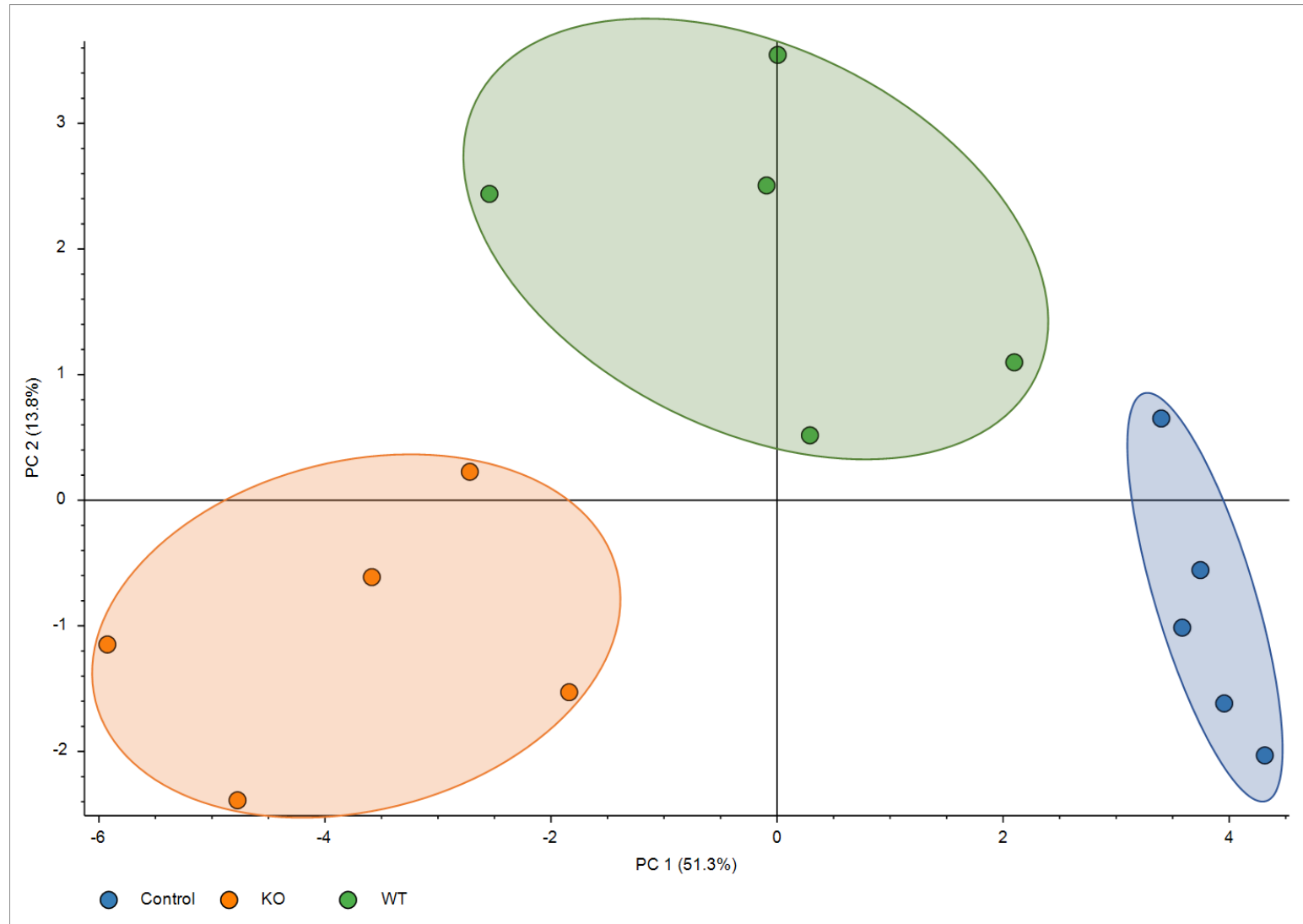

Figure 3. Principal component analysis plot showing separation between the three conditions used to challenge G. mellonella larvae. The control, easO knockout $M$. brunneum and wildtype $M$. brunneum separate into three different groups, suggesting different protein expression profiles. 


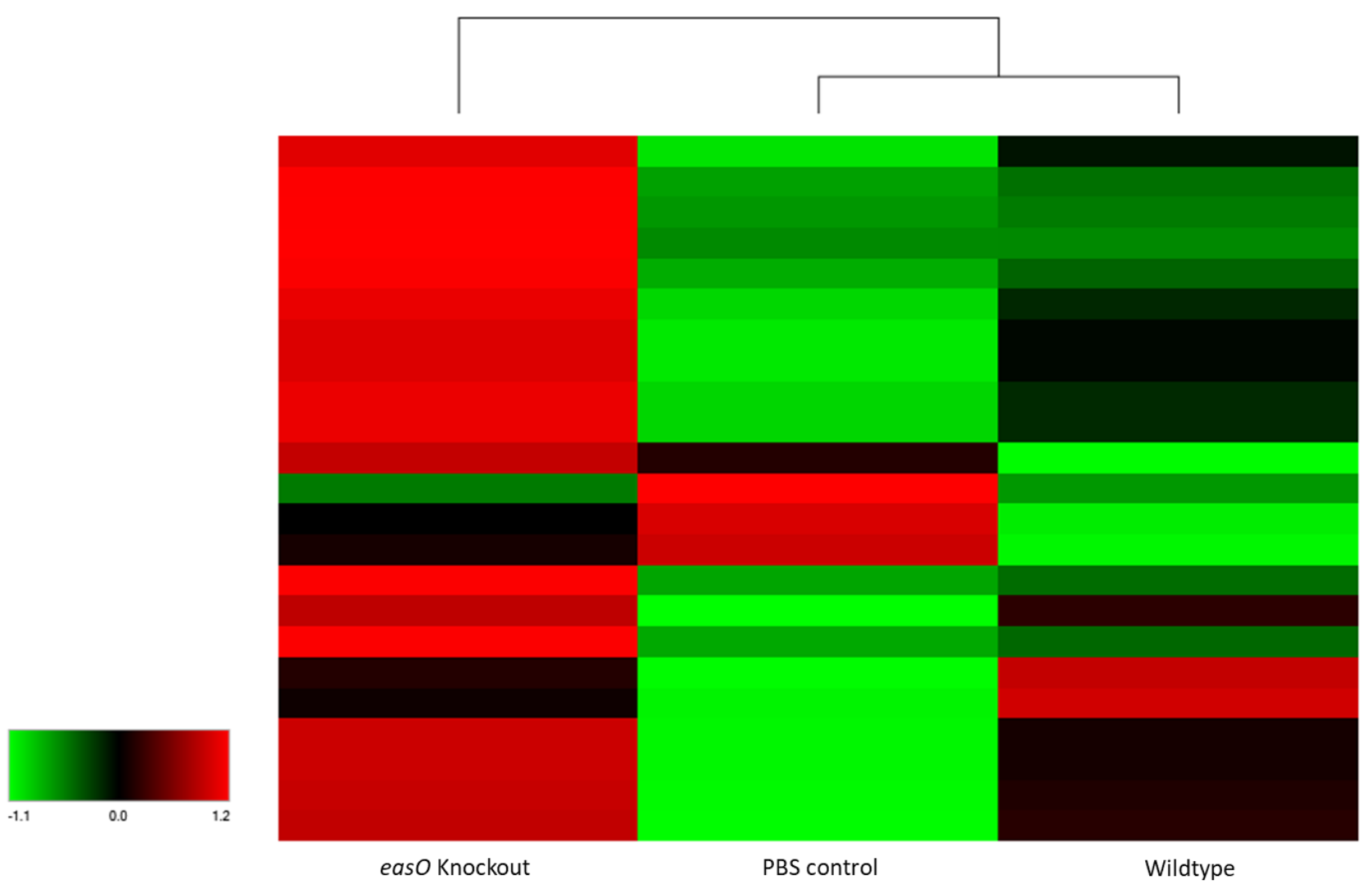

Figure 4. A Euclidean farthest neighbor hierarchical cluster generated from grouped protein abundances, scaled after clustering. These protein expression patterns indicate that general hemolymph protein content is more similar between the wild type infection and the PBS control than either is to the easO knockout infection. 

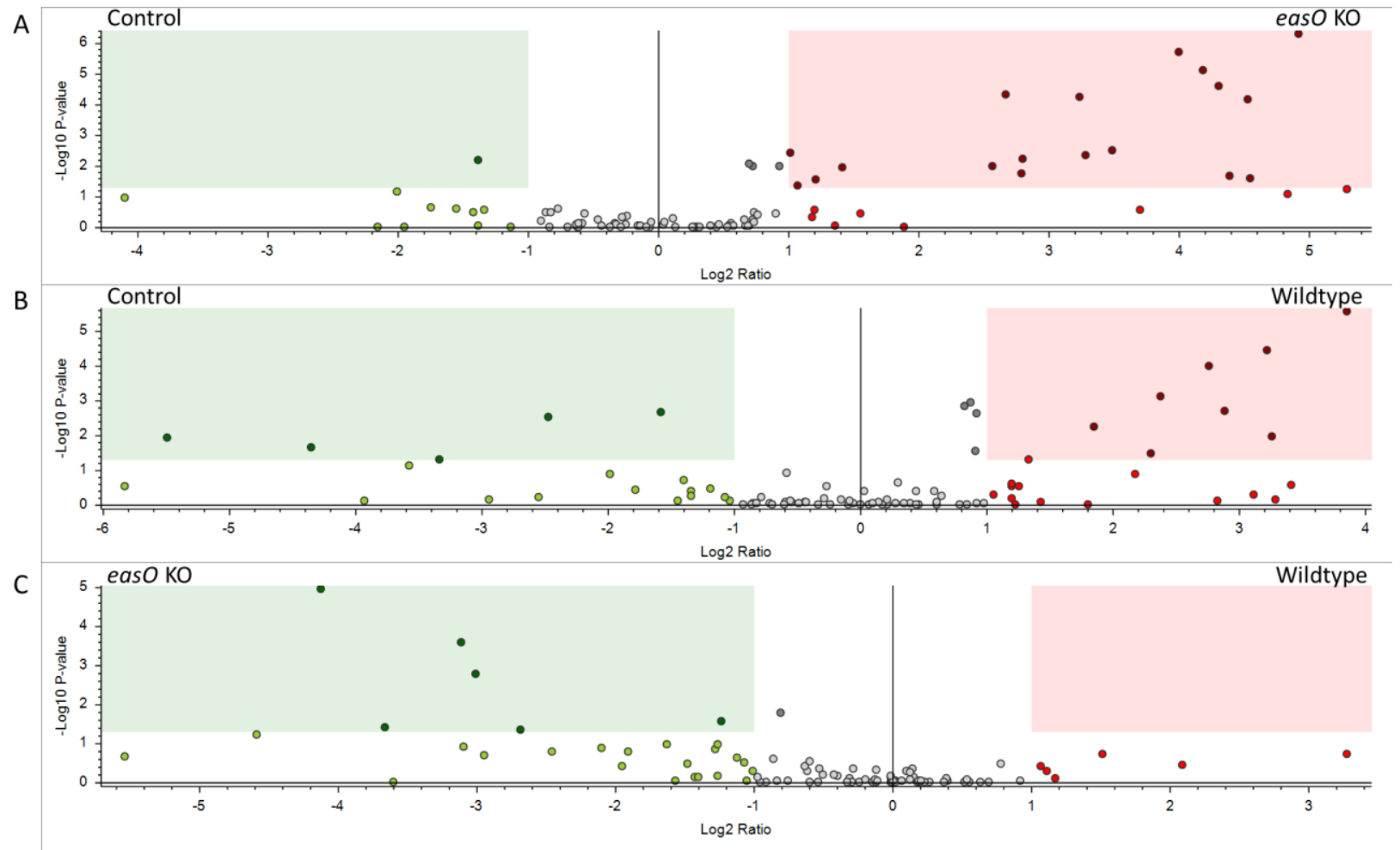

Figure 5. Volcano plots showing protein abundance differences in all identified proteins based on relative abundance changes in the three treatment types. Proteins are arranged based on their $p$ value ( $\log 10 \mathrm{p}$-value, $\mathrm{y}$-axis) and fold change ( $\log 2$ average label free quantitation difference in abundance, $x$ axis). Proteins found in the red and green boxes are considered to be statistically significant and having a log2 fold change greater than 1. 

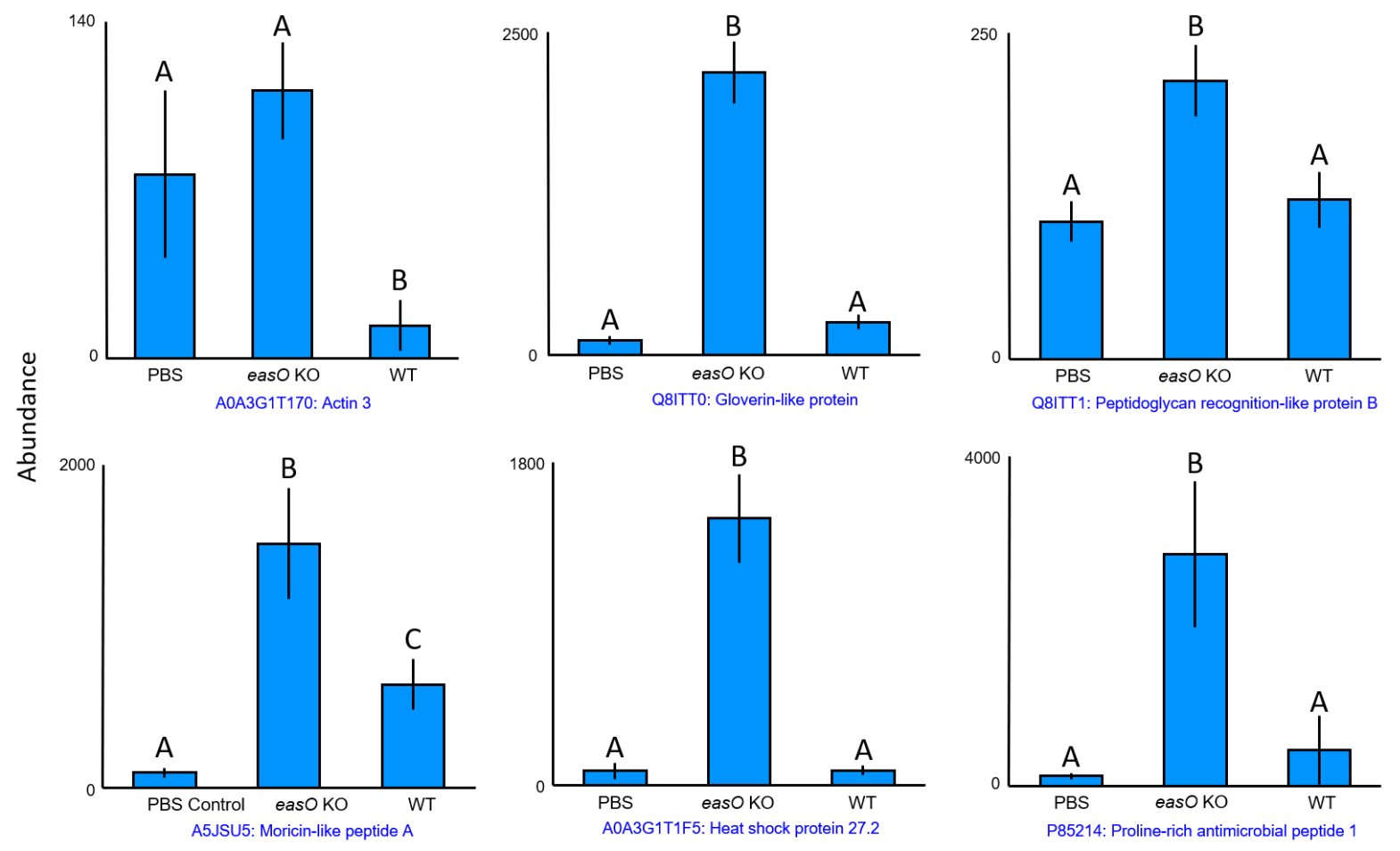

Figure 6. Bar graphs showing proteins that were differentially expressed between the easA knockout and wild-type strain infected G. mellonella. Moricin-like peptide A was the only protein which was differentially expressed between all three sample groups. 


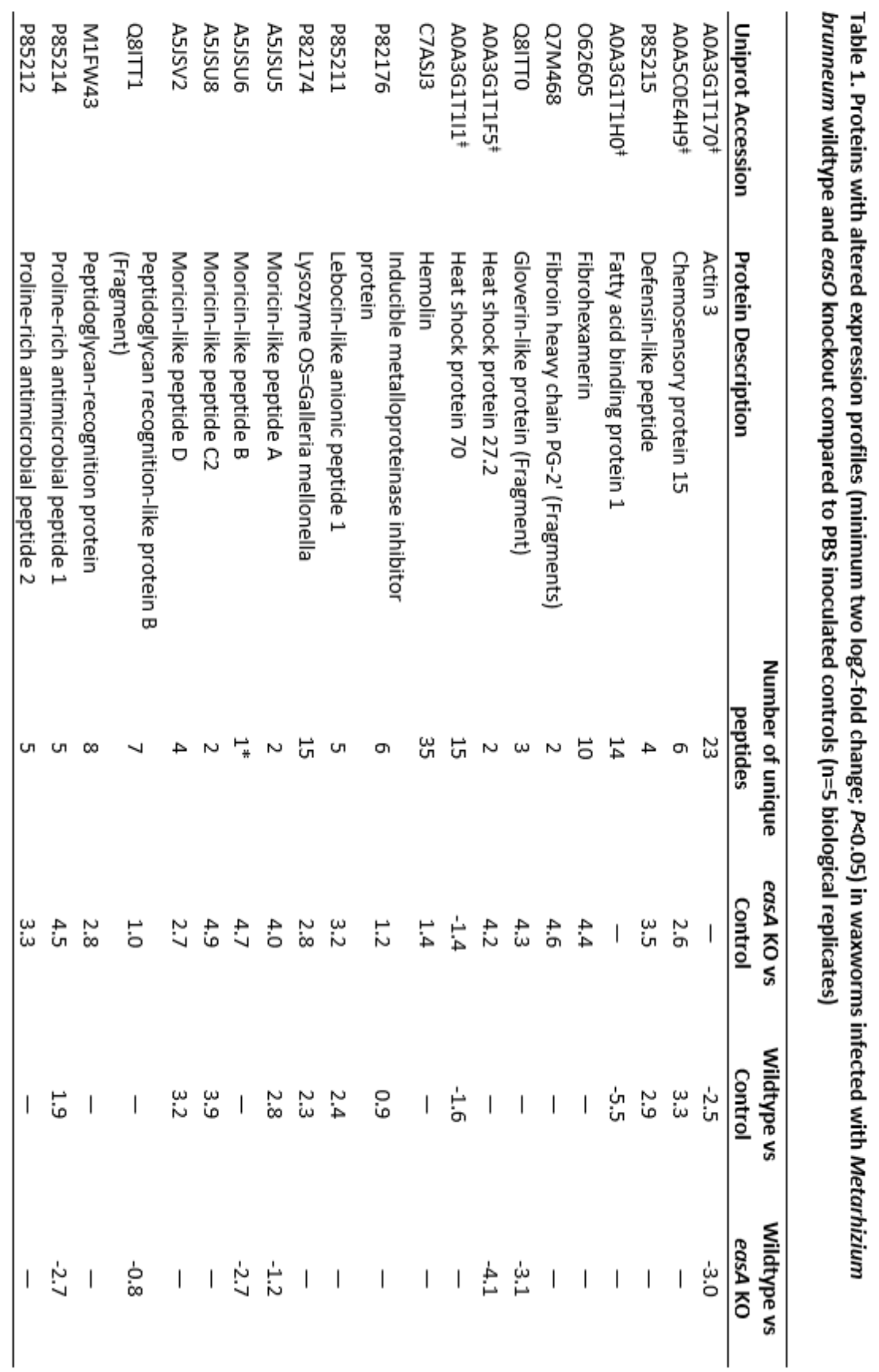


Table 2. Numbers of compounds that varied between Metarhizium species-infected corn roots compared to uninoculated corn roots by a minimum two log-fold difference $(P<0.05 ; n=3$ biological replicates).

Response $\quad$ Positive ionization mode Negative ionization mode

Up in M.b. ; up in M.f. ${ }^{b}$

${ }^{a}$ M.b., corn roots infected with M. brunneum ARSEF 9354

${ }^{b}$ M.f., corn roots infected with M. flavoviride BC 1163 
Table 3. Compounds altered in concentration (minimum two log-fold change; $P<0.05$ ) in corn roots infected with Metarhizium species compared to uninoculated corn roots ( $n=3$ biological replicates)

\begin{tabular}{|c|c|c|c|c|c|c|}
\hline $\begin{array}{l}\text { lonization } \\
\text { mode }\end{array}$ & Mass & $\begin{array}{l}\text { Log fold } \\
\text { change } \\
\text { M.b. }{ }^{a} \text { vs } \\
\text { Control }\end{array}$ & $\begin{array}{l}\text { Regulation } \\
\text { M.b. vs } \\
\text { Control }\end{array}$ & $\begin{array}{l}\text { Log fold } \\
\text { change } \\
\text { M.f. }{ }^{b} \text { vs } \\
\text { Control }\end{array}$ & $\begin{array}{l}\text { Regulation } \\
\text { M.f. vs } \\
\text { Control }\end{array}$ & Identification \\
\hline negative & 60.0232 & 15.9 & up & 14.9 & up & unknown \\
\hline negative & 103.0661 & 19.8 & up & 17.7 & up & Y-aminobutyric acid (GABA) \\
\hline negative & 114.0345 & 18.9 & up & 18.4 & up & $\begin{array}{l}\text { 4-hydroxy-5-methyl-3(2H)- } \\
\text { furanone }\end{array}$ \\
\hline negative & 138.0404 & -18.6 & down & -1.2 & unchanged & $\begin{array}{l}\text { salicylic acid or 4-nitroaniline } \\
\text { or 2-aminonicotinic acid }\end{array}$ \\
\hline negative & 150.0578 & 16.7 & up & 17.0 & up & unknown \\
\hline negative & 155.0729 & 20.7 & up & 20.3 & up & histidine \\
\hline negative & 166.0514 & -2.3 & down & -0.1 & unchanged & unknown \\
\hline negative & 166.0514 & 17.2 & up & 0.0 & unchanged & unknown \\
\hline negative & 177.0559 & 17.8 & up & 17.7 & up & unknown \\
\hline negative & 180.0287 & 17.6 & up & 18.6 & up & unknown \\
\hline negative & 181.0724 & 20.6 & up & 0.0 & unchanged & tyrosine \\
\hline negative & 188.0852 & 13.4 & up & 16.4 & up & unknown \\
\hline negative & 194.0447 & 16.1 & up & 0.0 & unchanged & 7-carboxy-7-deazaguanine \\
\hline negative & 199.1034 & 16.4 & up & 15.8 & up & unknown \\
\hline negative & 201.0675 & 14.9 & up & 15.3 & up & unknown \\
\hline negative & 205.0578 & 4.0 & up & 3.0 & up & unknown \\
\hline negative & 208.0941 & 14.3 & up & 15.8 & up & dambonitol \\
\hline negative & 215.0779 & 17.2 & up & 18.1 & up & succinyl proline \\
\hline negative & 239.0793 & 15.4 & up & 0.0 & unchanged & unknown \\
\hline negative & 240.0874 & 19.4 & up & 19.4 & up & unknown \\
\hline negative & 244.0654 & 3.4 & up & 3.1 & up & unknown \\
\hline negative & 250.0988 & 5.3 & up & 4.6 & up & $\begin{array}{l}\text { (3S,6S)-3-(4-Hydroxybenzyl)- } \\
6 \text {-(hydroxymethyl)-2,5- } \\
\text { piperazinedione }\end{array}$ \\
\hline negative & 254.0653 & 15.0 & up & 0.0 & unchanged & unknown \\
\hline negative & 266.1074 & 17.0 & up & 15.7 & up & unknown \\
\hline negative & 272.0684 & 18.6 & up & 0.0 & unchanged & unknown \\
\hline negative & 272.1166 & 17.4 & up & 15.8 & up & unknown \\
\hline negative & 279.1368 & 21.6 & up & 19.8 & up & $\begin{array}{l}\mathrm{N} \text {-(1-deoxy-1- } \\
\text { fructosyl)leucine }\end{array}$ \\
\hline negative & 306.0063 & 16.6 & up & 15.8 & up & unknown \\
\hline negative & 310.1848 & 19.3 & up & 0.0 & unchanged & potential botrydial \\
\hline negative & 317.1282 & 15.2 & up & 0.0 & unchanged & unknown \\
\hline
\end{tabular}




\begin{tabular}{|c|c|c|c|c|c|c|}
\hline negative & 326.1942 & 5.6 & up & 4.1 & up & $\begin{array}{l}\text { heptaethylene glycol or 2- } \\
\text { dodecylbenzenesulfonic acid }\end{array}$ \\
\hline negative & 354.1038 & -20.5 & down & -20.5 & down & asarinin \\
\hline negative & 358.0975 & 16.3 & up & 16.3 & up & unknown \\
\hline negative & 368.1164 & 15.0 & up & 14.2 & up & $\begin{array}{l}\text { 3-O-feruloyl-D-quinic acid (O- } \\
\text { feruloylquinic) }\end{array}$ \\
\hline negative & 388.1620 & 4.8 & up & -18.2 & down & unknown \\
\hline negative & 411.0667 & 16.3 & up & 0.0 & unchanged & unknown \\
\hline negative & 482.1381 & 18.2 & up & 0.0 & unchanged & unknown \\
\hline negative & 490.2749 & 2.4 & up & 0.0 & down & unknown \\
\hline negative & 502.3154 & -14.2 & down & -14.2 & down & unknown \\
\hline negative & 596.2847 & 23.7 & up & 23.9 & up & unknown \\
\hline negative & 688.4750 & 21.7 & up & 18.3 & up & unknown \\
\hline negative & 734.4921 & 3.3 & up & -1.6 & unchanged & unknown \\
\hline negative & 746.5172 & 2.9 & up & 2.2 & up & unknown \\
\hline negative & 810.6056 & 14.3 & up & 0.0 & unchanged & unknown \\
\hline negative & 812.6355 & 18.8 & up & 16.9 & up & unknown \\
\hline negative & 856.5146 & 4.9 & up & 4.0 & up & unknown \\
\hline positive & 124.0640 & 13.9 & up & 15.1 & up & 2-methoxy-3-methylpyrazine \\
\hline positive & 131.0945 & 20.6 & up & 19.7 & up & leucine/isoleucine \\
\hline positive & 131.1307 & 15.9 & up & 14.8 & up & unknown \\
\hline positive & 143.0945 & 20.2 & up & 17.6 & up & stachydrine \\
\hline positive & 173.1049 & 19.1 & up & 18.2 & up & $\mathrm{N}$-acetyl-L-leucine \\
\hline positive & 216.1108 & 17.5 & up & 17.7 & up & $\begin{array}{l}\text { tert-butyl 3-amino-1-methyl- } \\
\text { 2,3-dioxopropylcarbamate }\end{array}$ \\
\hline positive & 255.0745 & -17.8 & down & -17.8 & down & D-ribosylnicotinate \\
\hline positive & 276.0989 & 0.0 & unchanged & 15.2 & up & Glu-Glu \\
\hline positive & 283.1210 & -1.4 & unchanged & -16.6 & down & $\begin{array}{l}\text { ambiguous: ceramide or } n- \\
\text { trans- } p \text {-coumaroyl tyramine }\end{array}$ \\
\hline positive & 308.2238 & -1.5 & unchanged & -17.8 & down & $\begin{array}{l}\text { 7-(1-ethoxyethoxy)-4,10- } \\
\text { dimethyl-3,5,9,11- } \\
\text { tetraoxatridecane }\end{array}$ \\
\hline positive & 311.1219 & 14.8 & up & 15.2 & up & $\mathrm{N}, \mathrm{N}$-dimethylguanosine \\
\hline positive & 345.1224 & 0.0 & unchanged & 15.5 & up & unknown \\
\hline positive & 349.3177 & 14.7 & up & 0.0 & unchanged & unknown \\
\hline positive & 352.1310 & -13.7 & down & 0.3 & unchanged & $\begin{array}{l}\text { potential 1-(alpha-D- } \\
\text { glucopyranosyluronosyl)-3- } \\
\text { [(2S)-1-methyl-5-oxo-2- } \\
\text { pyrrolidinyl]pyridinium }\end{array}$ \\
\hline positive & 401.3491 & 1.8 & unchanged & -14.0 & down & unknown \\
\hline positive & 422.1051 & 15.9 & up & 17.2 & up & potential tetracenomycin $\mathrm{A} 2$ \\
\hline positive & 511.0720 & 25102.9 & up & 16.7 & up & unknown \\
\hline positive & 533.4801 & 36944.1 & up & 2.6 & up & unknown \\
\hline positive & 577.3516 & $\begin{array}{c}262910 . \\
0\end{array}$ & up & 19.5 & up & unknown \\
\hline
\end{tabular}




\begin{tabular}{ccccccc} 
positive & 603.2449 & 201676. & up & 0.0 & unchanged & unknown \\
& & 2 & & & & \\
positive & 911.6445 & 1.6 & unchanged & -14.5 & down & unknown \\
\hline
\end{tabular}




\section{CHAPTER 4 \\ INVESTIGATION OF EASC AND EXPRESSION OF THE ERGOT ALKALOID SYNTHESIS PATHWAY IN PENICILLIUM CAMEMBERTI}

\section{Abstract}

The Trichocomaceous fungus Penicillium camemberti is used in the production of camembert and brie cheeses. Ergot alkaloids are specialized metabolites synthesized by fungi belonging to the ascomycete families Trichocomaceae and Clavicipitaceae. The ergot alkaloid synthetic (EAS) pathway of fungi from Trichocomaceae (genera include Penicillium) diverges from fungi in Clavicipitaceae at a middle step to produce fumigaclavines and related compounds. Penicillium biforme is a known producer of rugulovasine A/B, which have never been observed in Penicillium camemberti. Analysis of the genomes from $P$. camemberti and $P$. biforme revealed that the two species contain the same cluster of EAS genes, and both organisms express mRNA from these genes in specific culture conditions. Heterologous gene expression of $P$. camemberti eas $C$ in a Neosartorya fumigata eas $C$ knockout strain, along with LC-MS analysis of $P$. biforme and $P$. camemberti cultures were used to test the regulation and the gene function of $P$. camemberti eas $C$. When grown on succinate and glucose containing media $P$. biforme produced rugulovasines, while the $P$. camemberti EAS pathway stopped at the step prior to Eas $C$ and accumulated $\mathrm{N}$-Me-dimethylallyltryptophan. Along with mRNA expression of eas $C$, this confirms that the regulatory elements needed for eas gene expression are functional in $P$. camemberti. When compared to $P$. biforme, $P$. camemberti eas $C$ contains a single nucleotide mutation. As a result, $P$. camemberti eas $C$ was unable to complement Neosartorya fumigata eas $C$ knockout in a transformed strain expressing the 
mRNA for the introduced gene. The data presented here suggest that the ancestor of modern $P$.

camemberti had the capacity to synthesize rugulovasines and other ergot alkaloid precursors but lost this capability due to a Gly to Val mutation at position 13 on the protein.

Some of the results presented in this chapter have been published as part of Fabian et al (2018). In this chapter, I have excerpted and expanded upon my original contributions to that published study. 


\section{$\underline{\text { Introduction }}$}

Ergot alkaloids are specialized metabolites synthesized by fungi belonging to the ascomycete families Trichocomaceae and Clavicipitaceae. The psychoactive, convulsive, and circulatory system effects of these compounds have been observed since the middle ages. In modern times, these compounds have been harnessed as precursors for medicines used to treat a variety of disorders including migraines, Parkinson's disease, and to prevent blood loss during childbirth (Haarmann et al., 2009). These effects are mostly attributed to lysergic acid related ergot alkaloids derived from Clavicipitaceae species, with clavine related compounds arising from Trichocomaceae being less understood (Florea et al., 2017).

Five enzymes involved in the early steps of the ergot alkaloid synthetic biochemical pathway are highly conserved across fungi and are contained in the ergot alkaloid synthesis (eas) gene cluster (Robinson \& Panaccione, 2015). Figure 1 shows a summary of these reactions. The gene dmaW encodes dimethylallyltryptophan (DMAT) synthase and begins the pathway with prenylation of tryptophan. Following this, the enzyme encoded by eas $F$ methylates the amino group of tryptophan, and a couple of reactions, involving enzymes coded by eas $C$ and easE, decarboxylate and oxidize the methylated DMAT and yield chanoclavine-I (Goetz et al., 2011; Lorenz et al., 2010). The primary alcohol on this closed-ring ergot alkaloid is then oxidized to an aldehyde by the product of the easD gene, yielding chanoclavine-I aldehyde (Wallwey et al., 2010). With this step, the conserved portion of the pathway is completed, with later products being modified forms of the chanoclavine-I aldehyde scaffold. Fungi from the Clavicipitaceae (genera include Metarhizium, Claviceps, Periglandula, Epichloë) perform modifications to form lysergic acid and related compounds, while fungi from Trichocomaceae diverge to produce fumigaclavines and related compounds (genera include Aspergillus and Penicillium) (Young et al., 2015). 
The genome sequence of the Trichocomaceous fungus Penicillium camemberti, used in the production of camembert and brie cheeses, was recently made publicly available (Cheeseman et al., 2014; Ropars et al., 2015). Analysis of its genome revealed that $P$. camemberti contains a cluster of ergot alkaloid synthesis (eas) genes. Interestingly, the closely related, ancestral species $P$. biforme contains a similar cluster organized in the same manner. Furthermore, the eas cluster from both species contain several genes with homology to the Neosartorya fumigata eas gene cluster (dmaW, easF, easC, easE, and easD), along with two additional genes, eas $H$ and eas $Q$ encoding activities associated with specialized metabolites (Fabian et al., 2018). A comparison of the $N$. fumigata and $P$. camemberti gene clusters can be seen in figure 2 .

Attempts by Fabian et al. (2018) to screen P. biforme and P. camemberti cultures using fluorescence (FLD) HPLC for the presence of ergot alkaloids were unsuccessful. Following successful transformation of chanoclavine-I aldehyde accumulating $N$. fumigata knockout strains with $P$. camemberti easH/easQ, cultures were screened by LC-MS and by FLD-HPLC for the presence of ergot alkaloids. LC-MS analysis revealed the presence of a $269.1 \mathrm{~m} / \mathrm{z}$, with high abundance counts in the mass spectrometer, and no signal in the FLD-HPLC analysis (excitation/emission wavelengths of $272 \mathrm{~nm} / 372$ $\mathrm{nm}$ and $310 \mathrm{~nm} / 410 \mathrm{~nm}$ ). While $269.1 \mathrm{~m} / \mathrm{z}$ is consistent with lysergic acid, the lack of FLD-HPLC signal hinted at a different structure. Indeed, LC-MS analysis of $P$. biforme malt extract cultures yielded a similar precursor mass and fragmentation spectra as that found in the $P$. camemberti easH/eas $Q$ transformed N. fumigata knockout strain (figure 3). P. biforme is a known producer of rugulovasines $A / B$, which have an accurate mass of $268.1206 \mathrm{Da}$ and is consistent with the observed $269.1 \mathrm{M}+\mathrm{H}$ ion observed.

Fabian et al. (2018) investigated and found by LC-MS analysis that ergot alkaloids were produced by $P$. biforme and not $P$. camemberti, when grown on malt extract agar. Furthermore, both Penicillium species produced mRNA transcripts for all the eas genes in the cluster. A heterologous 
complementation strategy along with $N$. fumigata eas gene knockout strains was used to investigate the functionality of several genes from the P. camemberti eas gene cluster. This work revealed that easH, eas $Q, d m a W$ and easE complemented $N$. fumigata knockout strains. By deduction and by chemical analyses described herein, I hypothesized that $P$. camemberti eas $C$ contains a defect, as a result this fungus has lost the ability to produce ergot alkaloids downstream of the easC gene product. Heterologous gene expression of $P$. camemberti in a N. fumigata easC knockout strain, along with LC-MS analysis of $P$. biforme and $P$. camemberti cultures were used to test this hypothesis.

\section{Methods}

\section{Culture conditions and ergot alkaloid extraction}

Petri dish cultures of P. biforme (NRRL 885), P. camemberti (NRRL 874 and 875), and N. fumigata easC knockout (Goetz et al., 2011) were grown on malt extract agar composed of $6 \mathrm{~g}$ of malt extract, $6 \mathrm{~g}$ dextrose, $1.8 \mathrm{~g}$ maltose, $1.2 \mathrm{~g}$ yeast extract, $15 \mathrm{~g}$ agar, totaling to $1 \mathrm{~L}$ with water), for 7 days at $22^{\circ} \mathrm{C}$. Cultures were also grown in a modified Czapek's medium (2 g sodium nitrate, 1 g dipotassium phosphate, $0.5 \mathrm{~g}$ magnesium sulfate, $0.5 \mathrm{~g}$ potassium chloride, 0.01 ferrous sulfate, totaling to $1 \mathrm{~L}$ with water), supplemented with succinate, glucose, glycine or glycerol as sole carbon sources at $10 \mathrm{mM}$. Ergot alkaloids were extracted from agar-based petri dishes, by repeatedly washing the culture with 4 $\mathrm{mL}$ of methanol. A portion of these methanol washes was concentrated using a vacuum concentrator.

\section{Transformation gene construct formation}

Restriction digested fusion PCR products were inserted in plasmids, to be used for heterologous gene expression. Phusion 5x high-fidelity PCR buffer $(100 \mathrm{mM} \mathrm{KCl}, 20 \mathrm{mM}$ Tris- $\mathrm{HCl}, 1.5 \mathrm{mM} \mathrm{MgCl}$, 
buffered at pH 7.4; Thermo Scientific, Waltham, MA) was combined with distilled/deionized water (11 $\mu \mathrm{L})$, Phusion HF polymerase ( $0.5 \mu \mathrm{L}$, Thermo Scientific), $1.25 \mathrm{mM}$ deoxynucleoside triphosphates (4 $\mu \mathrm{L})$, $20 \mu \mathrm{M}$ each of forward and reverse primer (1.25 $\mu \mathrm{L}$ of both), template DNA ( $2 \mu \mathrm{L})$. The sequences used for the forward and reverse primers can be found in table 1 . The $P$. camemberti eas $C$ coding sequence was amplified with primer set 1 , and the $N$. fumigata easA-eas $G$ promoter was amplified with primer set 2. These products were then combined by a fusion PCR reaction using primer set 3 . The PCR reactions followed a temperature-cycling program which consisted of an initial denaturing step at $98^{\circ} \mathrm{C}$ for $30 \mathrm{~s}$, then 35 cycles of denaturing at $98^{\circ} \mathrm{C}$ for $15 \mathrm{~s}$, annealing at the prescribed temperature, and extension at $72^{\circ} \mathrm{C}$ for the prescribed length of time, with a final extension of $72^{\circ} \mathrm{C}$ for $60 \mathrm{~s}$. The annealing temperatures and extension times can be found in table 1. The resulting PCR products were purified using a Zymogen DNA Clean \& Concentrator kit (Zymo Research Corp., Irvine, CA).

The fusion PCR product was ligated into the PBCphleo plasmid obtained from the Fungal Genetics Stock Center (Kansas State University, Manhattan, KS). Ligation was performed using T4 DNA ligase (New England BioLabs, Ipswich, MA), and the product was transformed into Escherichia coli. The resulting plasmids were recovered using a Zippy plasmid miniprep kit (Zymo Research Corp., Irvine, CA). A portion was analyzed by agarose gel electrophoresis to confirm successful construct formation.

\section{N. fumigata recombinant DNA transformation}

The $N$. fumigata easA/easG promoter - P. camemberti eas $C$ fusion $\mathrm{pBCphleo} \mathrm{plasmid} \mathrm{was}$ transformed into the $N$. fumigata eas $C$ knockout strain according to established methods (Fabian et al., 2018, Bilovol and Panaccione, 2016). The recipient $N$. fumigata culture was incubated overnight in malt extract broth. The collected mycelia were incubated at $22^{\circ} \mathrm{C}$ for 2 hours in $15 \mathrm{~mL}$ of $0.7 \mathrm{M} \mathrm{NaCl}$ along with $40 \mathrm{mg}$ of lysing enzyme (Sigma-Aldrich, St. Louis, MO) and $1 \mathrm{~g}$ of VinoTastePro (Gusmer Enterprises 
Inc., Mountainside, NJ). The resulting protoplasts were filtered, washed by repeated centrifugation and resuspension, and then incubated with the $N$. fumigata promoter-P. camemberti eas $C \mathrm{pBCphleo}$ plasmid construct as described previously (Bilovol and Panaccione, 2016). Phleomycin-resistant transformed protoplasts were transferred to malt extract agar containing phleomycin. Single spore cultures were then selected, to ensure nuclear purity, and cultured for genotype confirmation by DNA extraction with PCR (using primer set 4 from table 1) and phenotype confirmation by LC-MS.

\section{mRNA extraction and CDNA generation}

Transformants containing- $P$. camemberti eas $C$ and that had been verified for their ergot alkaloid phenotype were re-cultured as mycelial mats in malt extract broth for 3 days at room temperature. Mats were extracted and flash frozen in liquid nitrogen and pulverized via mortar and pestle. A Qiagen RNeasy plant kit (Qiagen, Germantown, MD) was used to extract RNA from the cultures; DNA was removed using on-column Dnase I digestion. The resulting RNA sample was reverse transcribed using SuperScript IV reverse transcriptase (Thermo Scientific, Waltham, MA) and an oligo(dT) primer. A PCR reaction using primer set 4 from table 1 was used to amplify eas C cDNA, which was then analyzed by agarose gel electrophoresis for length. Sanger sequencing (Eurofins Genomics, Louisville, KY) was used to confirm the sequence of the PCR product.

\section{LC-MS analysis of ergot alkaloid extracts}

High-resolution mass spectra were collected on a Thermo Scientific Q Exactive mass spectrometer coupled to a Thermo Accela 1250 UHPLC system. The mass spectrometer was operated in positive ion mode using data dependent acquisition settings. Precursor scans were acquired at 70,000 
resolution (at $m / z=200$ ) over an 80 to $1200 \mathrm{~m} / z$ mass range. The 10 most abundant ions from each precursor scan were selected for HCD fragmentation $(\mathrm{NCE}=30)$ and were analyzed at 35,000 resolution. Separations were performed on a $2.1 \mathrm{~mm}$ by $100 \mathrm{~mm}$ Zorbax Eclipse XDB-C18 column (Agilent, Santa Clara, CA) using a gradient composed of mobile phase A (water, $0.1 \%$ formic acid) and mobile B (acetonitrile, $0.1 \%$ formic acid). The sample was loaded at $95 \% A+5 \% B$ and held for one minute before ramping linearly to $40 \% \mathrm{~A}+60 \% \mathrm{~B}$ at 20 minutes using a flow rate of $300 \mu \mathrm{L} / \mathrm{min}$.

Unit mass resolution spectra (figure 5) were collected on a Thermo LCQ Deca XP Plus mass spectrometer coupled to a Thermo Surveyor HPLC system (Thermo Scientific, Waltham, MA). The mass spectrometer was configured and operated in the manner according to what is described in Ryan et al. $(2013,2015)$. Separations were performed on a $2 \mathrm{~mm}$ by $150 \mathrm{~mm}$ Phenomenex 4- $\mu \mathrm{m}$ polar RP column using a gradient composed of mobile phase $A$ ( $5 \%$ acetonitrile, $0.1 \%$ formic acid) and mobile phase $B$ (75\% acetonitrile, $0.1 \%$ formic acid) at $200 \mu \mathrm{l} / \mathrm{min}$. Samples were loaded at $14 \% \mathrm{~B}$, and eluted with a linear gradient which ramped to $100 \%$ B over 20 minutes.

\section{Results}

\section{P. camemberti eas pathway is expressed under certain culture conditions}

Investigation into culture conditions of $P$. camemberti and $P$. biforme revealed differences in ergot alkaloid production. Fabian et al. (2018) demonstrated that $P$. camemberti eas genes were transcribed when the organism was grown on malt extract agar, but not when grown in conditions used for cheese making. To test for differences between $P$. biforme and $P$. camemberti nutritional regulation of the eas pathway, both species were grown on a modified Czapek's media, and supplied alternately with glucose, succinate, glycerol and glycine as sole carbon sources. Those $P$. biforme cultures grown on glucose and succinate containing media (but not the other listed carbon sources) produced 
rugulovasines, whereas $P$. camemberti cultures were not able to produce rugulovasines under any culture conditions. However, under conditions which induced rugulovasines in P. biforme, P. camemberti produced N-methyl DMAT (figure 4, figure 5). Cheese samples (commercial and home-made) were analyzed for N-Me-DMAT, but none was detected.

\section{Rationale for investigating easC}

Fabian et al. (2018) showed that three eas genes of $P$. camemberti (dmaW, easC, easE) had genetic polymorphisms when compared to functional homologs from P. biforme. Moreover, they showed, by heterologous expression of $P$. camemberti eas genes in the corresponding $N$. fumigata knockout strains, that $P$. camemberti eas $H$, eas $Q$, easE and $d m a W$ complemented or augmented (in the case of easH and eas $Q$ ) the corresponding $N$. fumigata mutant and thus encode functional enzymes. Furthermore, it has been demonstrated that eas pathway regulation is intact for $P$. camemberti under the correct culture conditions (figure 4, figure 5), as evidenced by the presence of N-Me-DMAT and gene transcripts for eas genes. It can be reasoned that there must exist a defect in a gene downstream of $\mathrm{N}$ Me-DMAT in P. camemberti. Since the product of easC uses N-Me-DMAT as a substrate, I hypothesized that easC of $P$. camemberti (which had not previously been functionally analyzed but did contain a SNP relative to the functional allele in P. biforme) was dysfunctional (figure 6).

\section{P. camemberti easC does not restore N. fumigata easC knockout strain's mutant phenotype}

A construct for expressing easC in N. fumigata was prepared by joining the promoter from the $N$. fumigata eas $A$ gene to the coding sequences and $3^{\prime}$ untranslated sequences of $P$. camemberti eas $C$ (figure 7). When a previously constructed $N$. fumigata easC knockout strain (Goetz et al., 2011) was 
transformed with the $N$. fumigata eas $A$ promoter $+P$. camemberti eas $C$ fusion construct, fumigaclavine C was not produced. Figure 8 shows the results of a PCR experiment using promoter combination 4 in table 1 , where a fragment of genomic DNA was amplified, matching the size of the $P$. camemberti eas $C$ construct (1391 bp). Sanger sequencing of this product confirmed the identity of the amplified gene, verifying that the gene had been inserted into the $N$. fumigata eas $C$ knockout strain (figure 8).

\section{N. fumigata transcribes and correctly splices the $P$. camemberti eas $C$ gene}

Figure 6 shows the predicted location of introns and exons for $P$. camemberti eas $C$. The $P$. camemberti eas $C$ genomic sequence was aligned with eas $C$ from $N$. fumigata and the allele from another well-characterized ergot alkaloid producer, Claviceps purpurea (figure 9). The overall layout of the eas $C$ gene is similar among the three species. To confirm that $P$. camemberti eas $C$ was transcribed and properly processed by $N$. fumigata, a reverse transcription-PCR experiment was performed to verify the presence of correctly spliced mRNA corresponding to $P$. camemberti eas $C$. Figure 8 shows RT-PCR analysis using primers for $P$. camemberti easC. This analysis revealed a cDNA amplification product with a reduction in length corresponding to the predicted size of the intron (68 bp). Correct splicing of the intron was confirmed by Sanger sequencing of the cDNA. Shown in figure 8 is the absence of the intron with the two exons adjacent to one another in the cDNA. These results indicate that the $P$. camemberti gene was transcribed and spliced in a manner that was predicted and is consistent with how N. fumigata and C. purpurea splice their easC genes. 


\section{A point mutation in $P$. camemberti eas $C$ causes it to lose function}

Also confirmed by these Sanger sequencing results (figure 10), is a point mutation of $G \rightarrow T$ at position 199 of the gene. This results in a GTC codon instead of a GGC codon and causes $P$. camemberti to express valine at position 13 instead of the glycine residue at the same position in the homologous protein from $P$. biforme. Amino acid alignments of eas $C$ homologs from $P$. biforme, N. fumigata, $C$. purpurea, Periglandula ipomoeae, Metarhizium anisopliae and Epichloe inebriens reveal a conserved polar amino acid residue (GIn, Lys, or Asp) in that position for the other species surveyed (figure 11). $P$. biforme eas $C$ codes for a Gly residue at that position, with $P$. camemberti coding for a Val residue. Since this mutation was the only difference between the alleles from P. camemberti and P. biforme, and because $P$. camemberti accumulated the substrate for EasC (N-Me-DMAT), these results indicate that the mutation of a Gly to a Val residue causes the $P$. camemberti gene to lose the ability to catalyze decarboxylation of methylated DMAT to yield the precursor to chanoclavine-I. While, N-Me-DMAT is not the direct substrate for EasC, the substrate is likely the oxidized diene product of EasE (284 Da). This compound was not directly observed, it was proposed that EasE and EasC form a complex, so that the oxidized diene of N-Me-DMAT is a transient form (Goetz et al., 2011).

\section{Discussion}

The inability of $P$. camemberti eas $C$ to restore the $N$. fumigata eas $C$ knockout strain's production of ergot alkaloids correlates well with the LC-MS and transcriptome results previously reported (Fabian et al., 2018; Lessard et al., 2014). Fabian et al. detected no ergot alkaloids of any kind in cheese cultures of $P$.camemberti and $P$. biforme of varying ages and sources. Also detected were $P$. camemberti gene transcripts for eas genes in certain culture conditions but not in cheese cultures. While $P$. camemberti 
cannot synthesize rugulovasines, the presence of N-Me-DMAT suggests that regulatory elements needed for eas gene expression are still functional. Furthermore, there is a nutritional regulatory element regarding the expression of eas genes in both Penicillium sp. studied. The fact that succinate and glucose were the only carbon sources that allowed the fungi to produce ergot alkaloids suggests regulatory elements related to the citric acid cycle (succinate enters metabolism as a citric acid cycle intermediate). Most importantly, the data suggest that eas $C$ is defective in P. camemberti.

Residue 13 of EasC from both Penicillium spp. differs from the conserved polar amino acid residues seen in other ergot alkaloid synthesizing fungi. Considering this, it is noteworthy that only $P$. biforme (as opposed to P. camemberti) retained a functional eas $C$ gene. Betts and Russell (2007) discuss the consequences of substituting amino acid residues - while substituting polar residues carries little to no penalty in most cases, substitution of a hydrophobic valine residue generally carries a relatively harsh penalty (as in $P$. camemberti). Due to the bulky side chain on valine, this residue can have a restrictive impact on protein backbone folding conformations. In the case of $P$. biforme, the presence of a substituted glycine may impact activity if it occurs at the catalytic site, but otherwise with regards to structure it contributes little more than flexibility due to its lack of side chain. This mutation likely modifies the higher-order structure of the protein in such a way as to impact the catalytic site or to modify the required interaction of EasC with some other protein (e.g. EasE).

The data presented here suggest that the ancestor of modern $P$. camemberti had the capacity to synthesize rugulovasines and other ergot alkaloid precursors. While there is a lack of information regarding the toxicity of rugulovasines, they have been shown to be deadly to day-old poultry and to decrease blood pressure of cats (Meurant, 1981). Clearly the presence of these compounds in cheese would be a public health concern. Fortunately, we have shown that two factors prevent the accumulation of these compounds from $P$. camemberti, the fungus used to ripen camembert cheese., 
Due to its use in controlled domesticated processes, and that the end cheese product was destined for human consumption, there likely was a lack of selective pressure for $P$. camemberti to maintain eas $C$. 


\section{Figures}
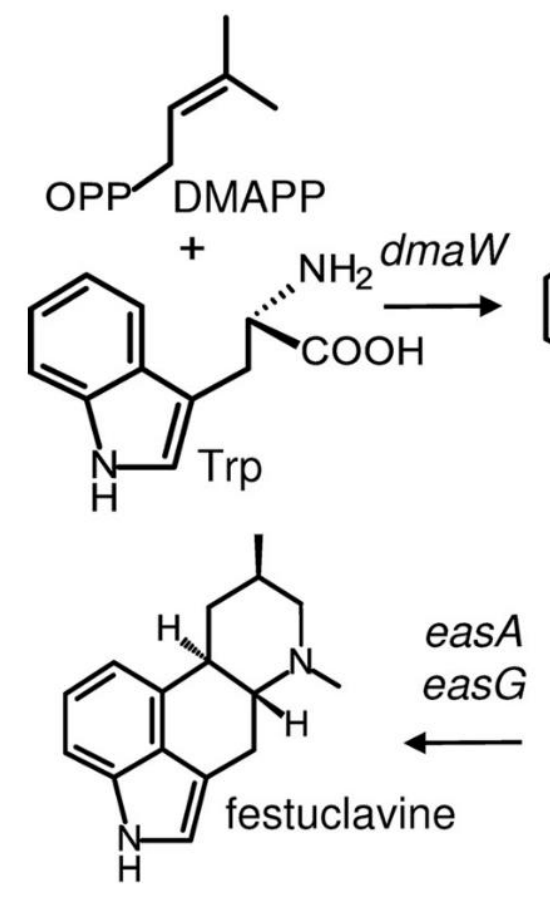<smiles>CC(C)=CCc1cccc2[nH]cc(C[C@H](N)C(=O)O)c12</smiles><smiles>CN[C@@H](Cc1c[nH]c2cccc(CC=C(C)C)c12)C(=O)O</smiles>

Figure 1. The ergot alkaloid synthesis pathway found in Neosartorya fumigata. The steps catalyzed by $d m a W, e a s F, e a s C, e a s E$, and eas $D$ are conserved across many fungi. These genes are found in Penicillium camemberti and Penicillium biforme, which diverge after the easD step. This figure was originally presented by Fabian et al. (2018). 


\section{A Penicillium camemberti eas cluster easD dmaW easE easF easC easH eas $Q$

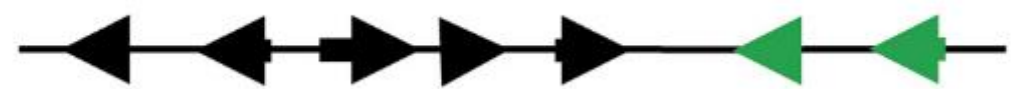

\section{B Neosartorya fumigata eas cluster}

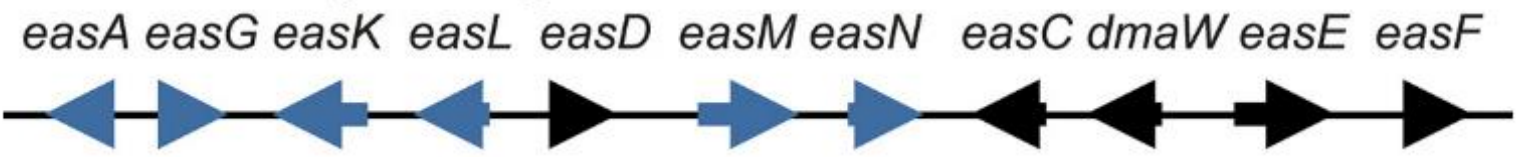

Figure 2. The alignment of ergot alkaloid synthesis genes between $P$. camemberti and $N$. fumigata. The

first five genes are conserved between N. fumigata, P. biforme, and P. camemberti (shown in black),

while two genes are unique (shown in green) to $P$. camemberti and six genes (shown in blue) are unique to N. fumigata. This figure was originally presented by Fabian et al. (2018). 


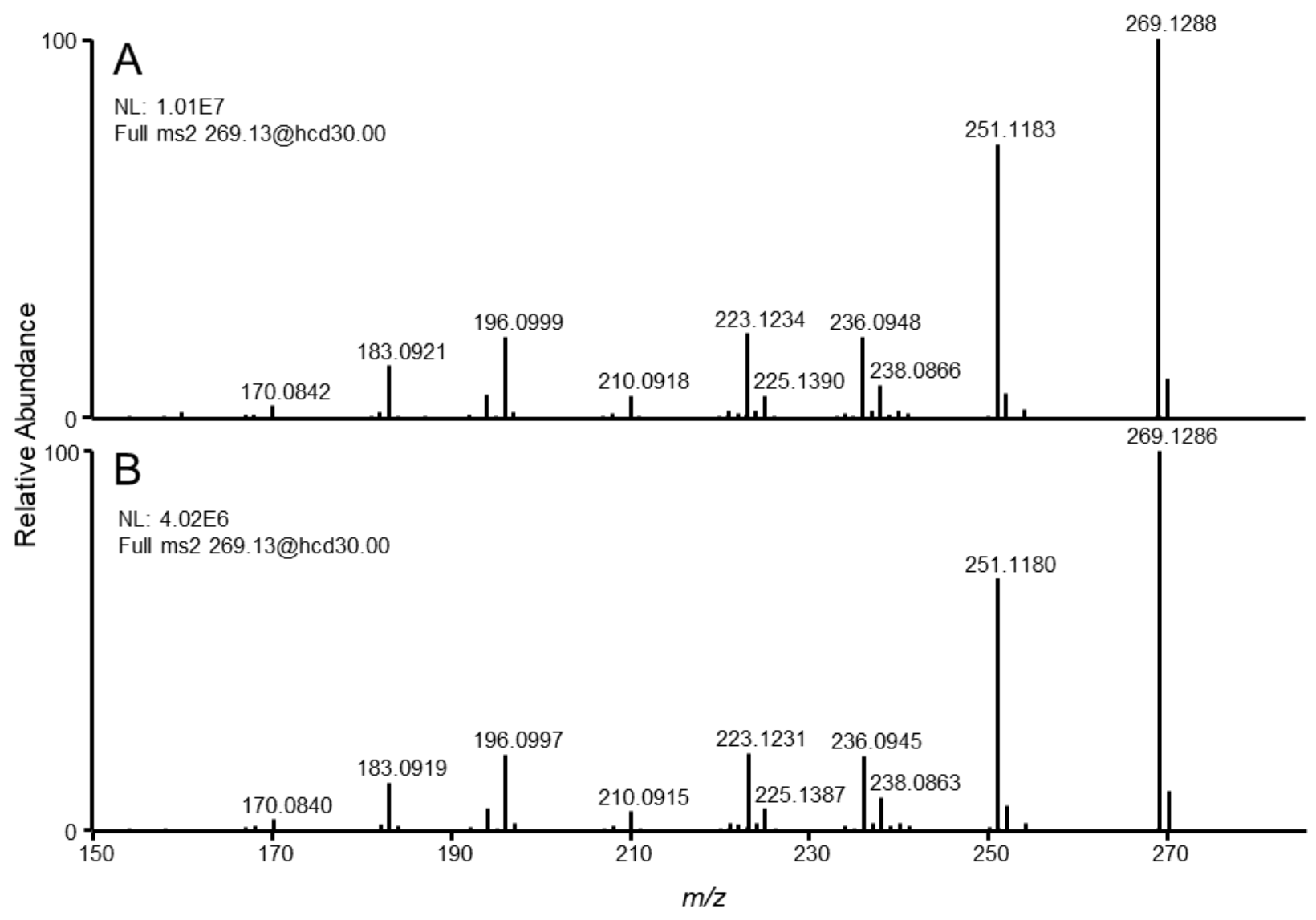

Figure 3. High-resolution mass spectra of ions resulting from fragmentation of the parent ion with a mass consistent with rugulovasine $(269.13 \mathrm{~m} / \mathrm{z})$ in (A) easH/eas Q transformed $N$. fumigata easA knockout and (B) P. biforme. Data were collected on a Thermo Q Exactive mass spectrometer operated in positive mode. 


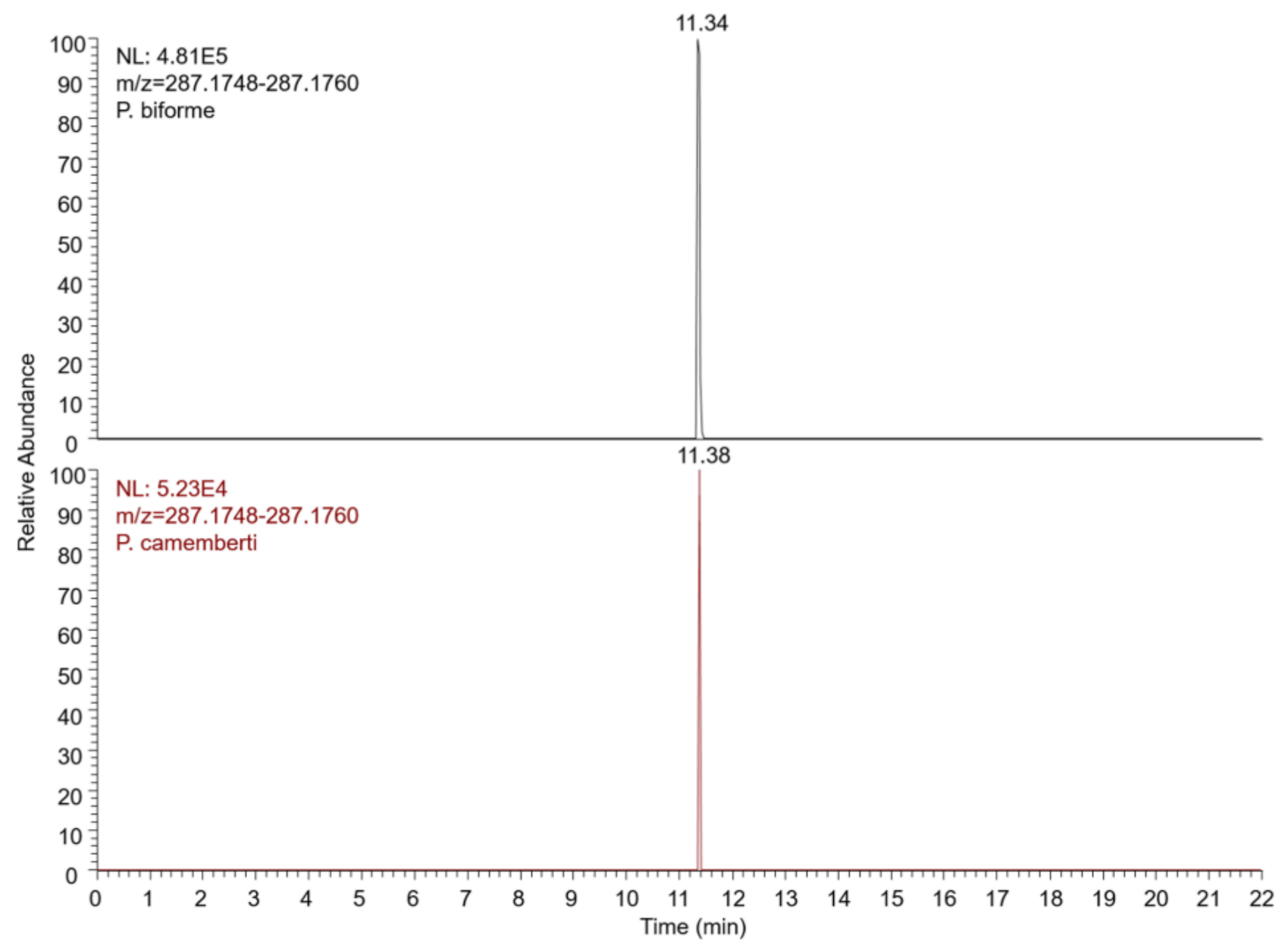

Figure 4. The presence of N-Me-DMAT in vitro from $P$. biforme and $P$. camemberti cultures. The chromatogram for $287.1754 \mathrm{~m} / \mathrm{z}$ was extracted using a $2 \mathrm{ppm}$ mass error extraction window. These data were generated using a Thermo Scientific Orbitrap high resolution accurate mass spectrometer. 


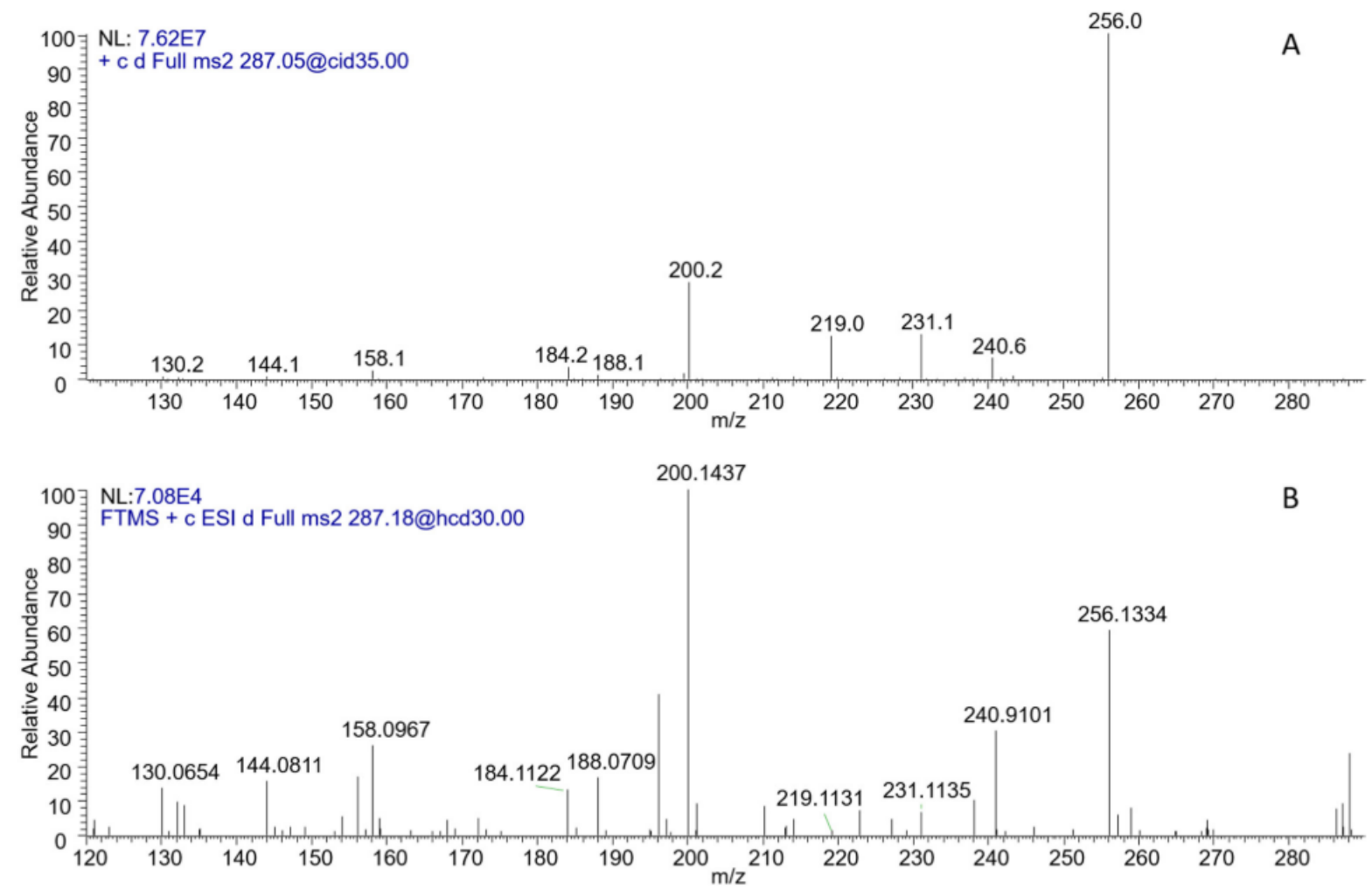

Figure 5. A CID fragmentation spectrum, of parent ion $287.1 \mathrm{~m} / \mathrm{z}$, was previously acquired from a N-MeDMAT standard, using a Thermo Scientific ion trap mass spectrometer (A). The resulting HCD MS/MS spectrum of the extracted ion $(287.1754 \mathrm{~m} / \mathrm{z})$ at 11.3 minutes from figure 4 , generated on a Thermo Scientific Orbitrap mass spectrometer (B). 


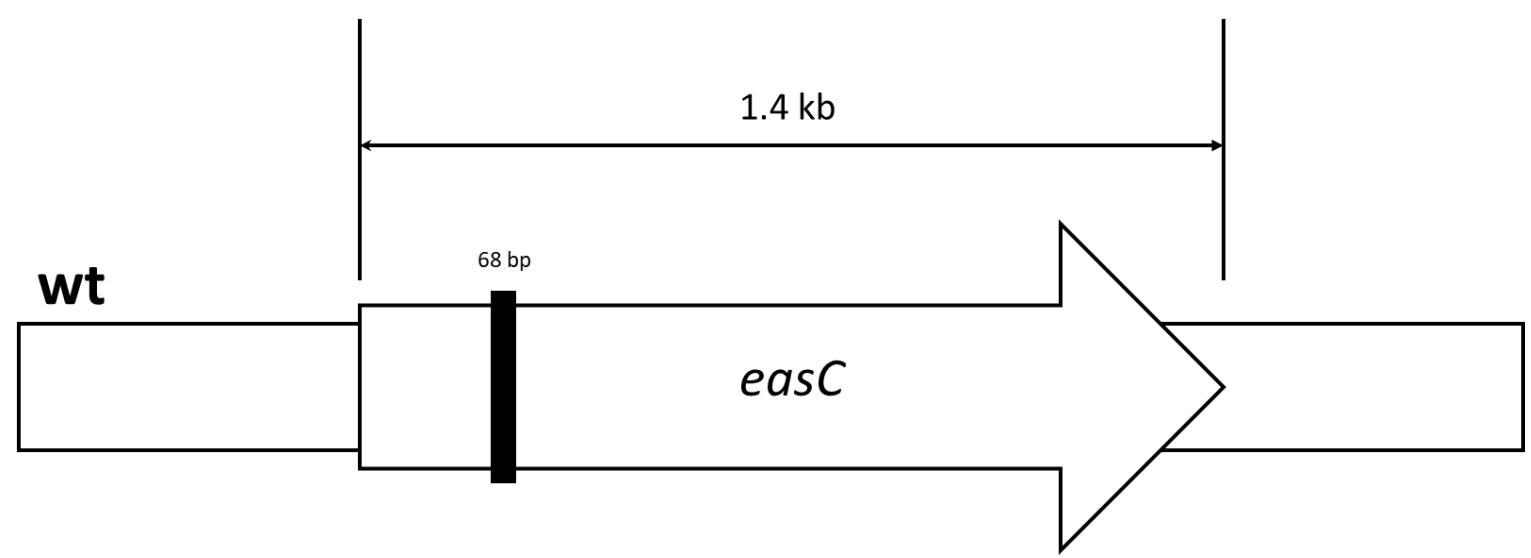

Figure 6. The ergot alkaloid synthesis gene cluster in $P$. camemberti and $P$. biforme contain the eas $C$ gene the product of which, along with the product of easE, decarboxylates methylated DMAT to yield chanoclavine-I. The gene is approximately $1.4 \mathrm{~kb}$ long, and contains a putative intron (marked with black) of $68 \mathrm{bp}$. 

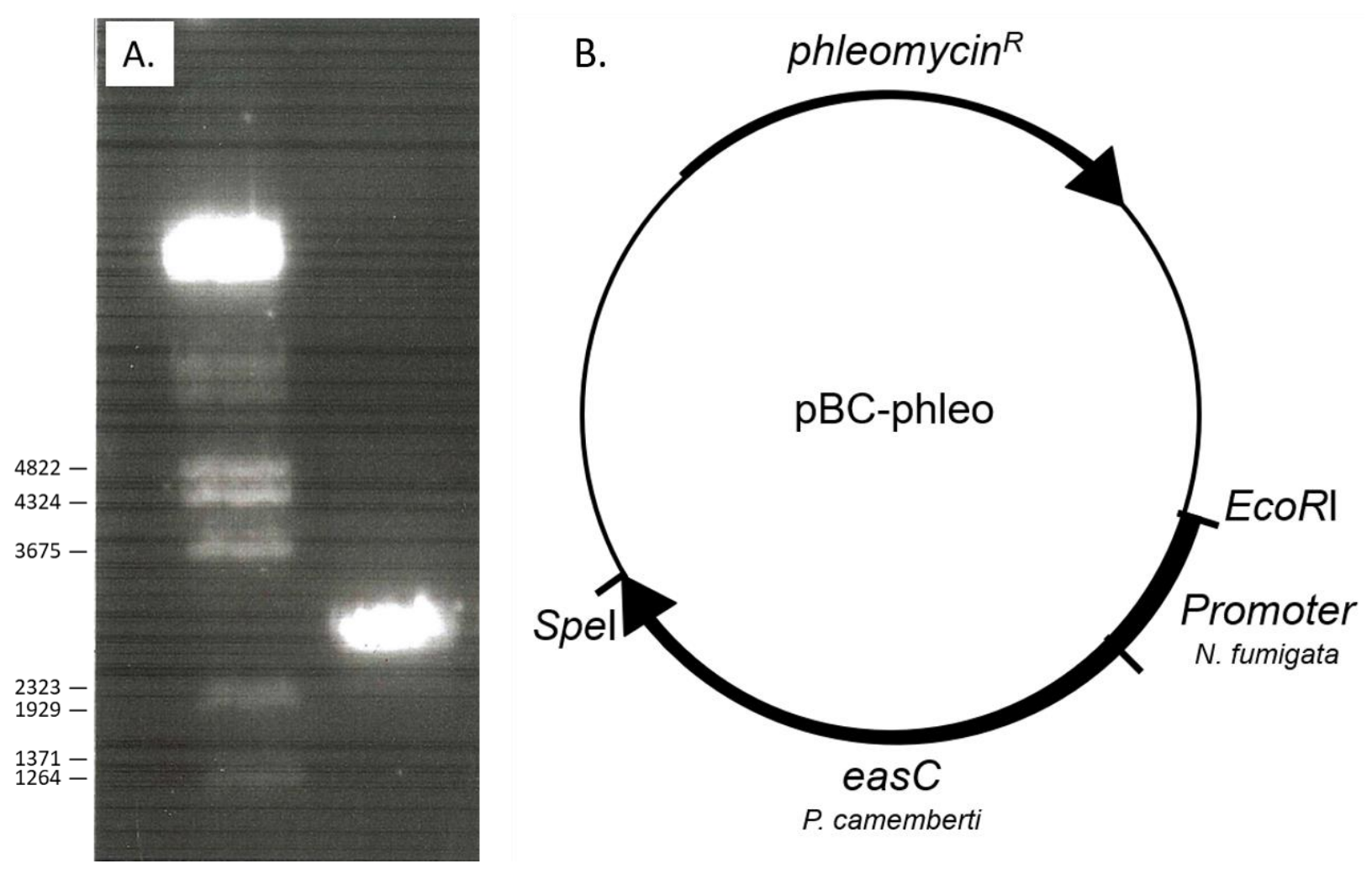

Figure 7. The easA-easG promoter from $N$. fumigata is joined to the $P$. camemberti eas $C$ gene by fusion PCR (A). The resulting product was inserted in the $\mathrm{pBCphleo}$ plasmid as a vector to complement the $N$. fumigata easC KO strain (B). 


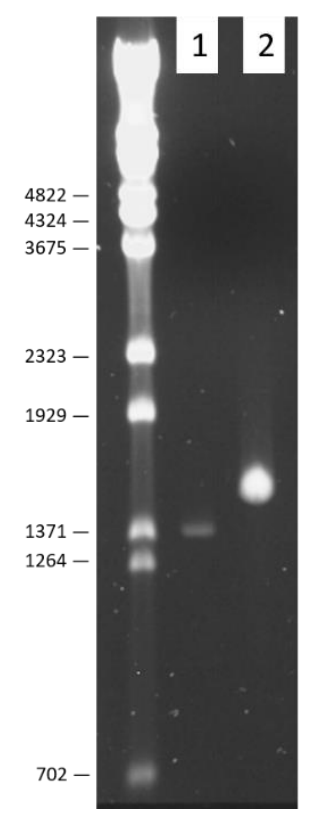

Lane 1

Sense strand

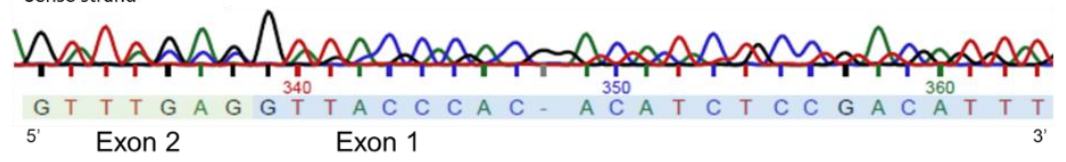

Lane 2

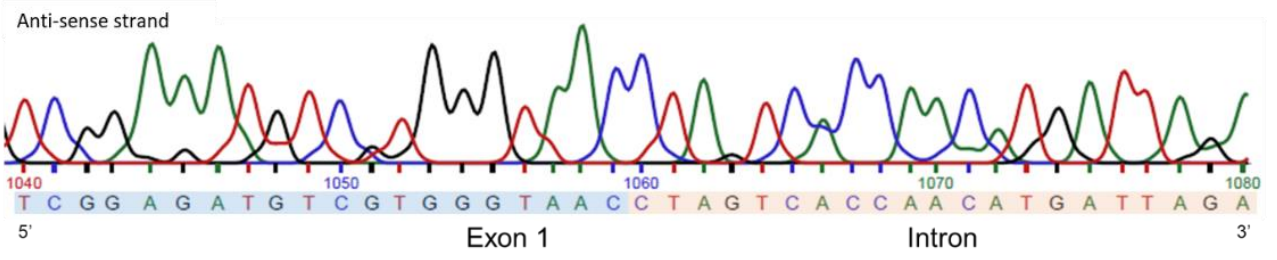

Figure 8. The same primers for $P$. camemberti eas $C$ were used to amplify cDNA (lane 1 ) and genomic

DNA (lane 2) from $N$. fumigata eas $C$ knockout transformed with the $P$. camemberti eas $C$ gene. Agarose electrophoresis of the two PCR products shows that the cDNA product is shorter than the genomic DNA product. Sanger sequencing of the product in lane 1 shows exon 1 (blue) directly adjacent to exon 2 (green), while sequencing of the product in lane 2 shows exon 1 (blue) directly adjacent to the intron. 


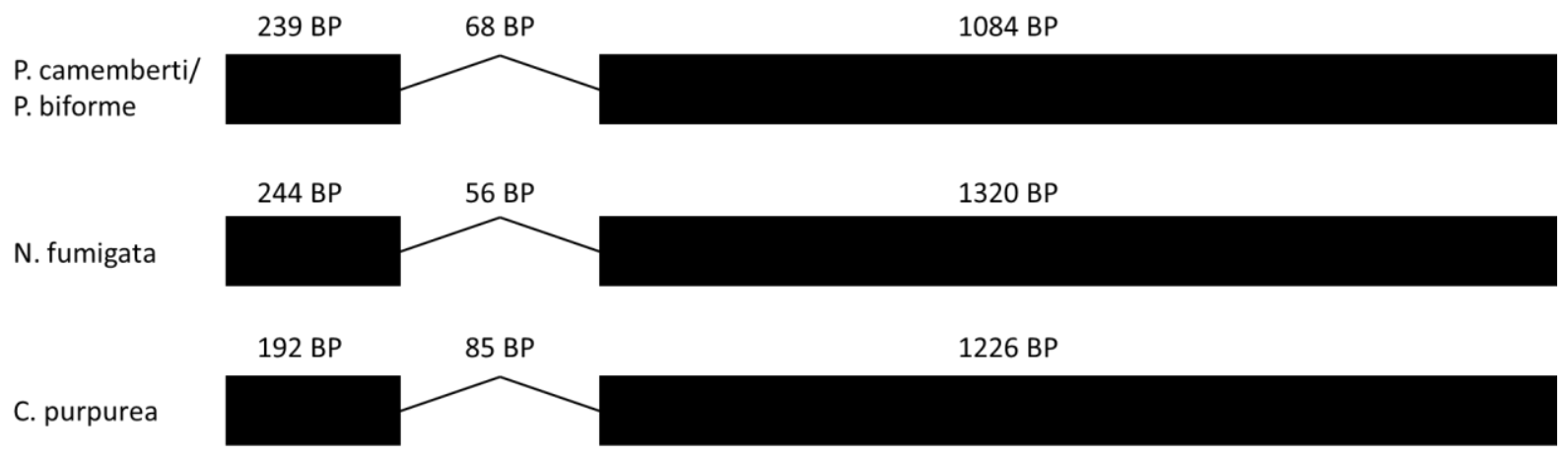

Figure 9. The splicing scheme for eas $C$ from $P$. camemberti is consistent with the splicing scheme from the homologous gene found in P. biforme, N. fumigata and C. purpurea. 


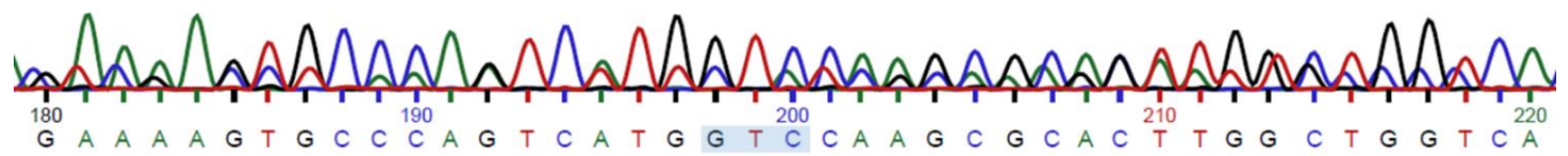

Figure 10. Sanger sequencing results of $P$. camemberti eas $C$ cDNA as expressed in $N$. fumigata eas $C$ knockout demonstrate a point mutation (highlighted in blue). This results in a GTC codon (valine) instead of a GGC codon (glycine) at that position. 


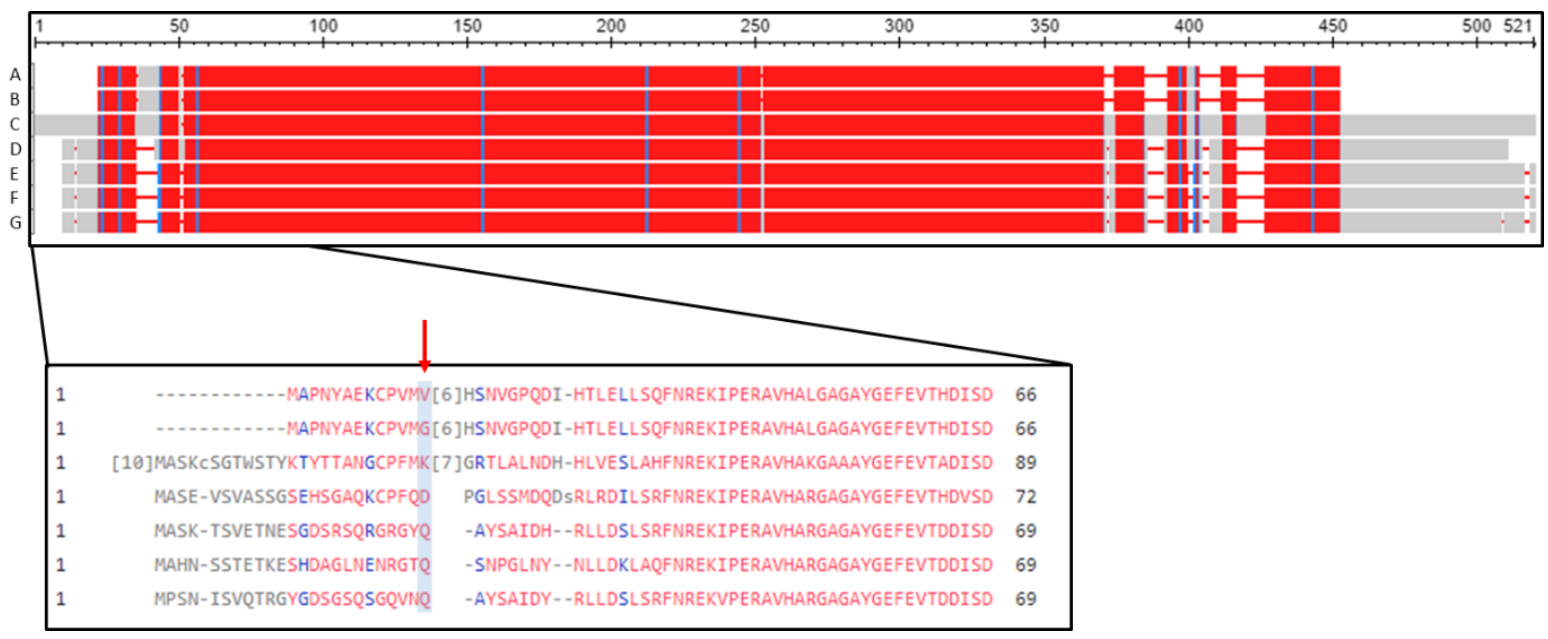

Figure 11. COBALT multiple protein sequence alignment of the eas $C$ gene from seven fungi - (A)

Penicillium camemberti, (B) Penicillium biforme, (C) Neosartorya fumigata, (D) Claviceps purpurea, (E)

Periglandula ipomoeae, (F) Metarhizium anisopliae, (G) Epichloë inebrians. Amino acid residues in red are conserved, while those in blue are not. The valine at position 13 in the $P$. camemberti sequence has been highlighted. This position is occupied by a large polar residue (>115 Da) in five of the seven species, while $P$. biforme has a small glycine $(57 \mathrm{Da})$ and $P$. camemberti has a valine which is larger (99 Da) and non-polar. 
Table 1. PCR primers and method

\begin{tabular}{clc}
\hline Primer Set & Primer sequence (5' to 3') & \multicolumn{1}{c}{$\begin{array}{c}\text { Product and length (bp) } \\
\text { Annealing temperature ('C), } \\
\text { Extension time (s) }\end{array}$} \\
\hline 1 & $\begin{array}{l}\text { GAGTAGGCACTCCGCACCATGATCTCAATAGATCATATTCC + } \\
\text { GTGGCCTTGAGTTAACTGAAGC }\end{array}$ & easC with $N$. fumigata promoter extension $(2,098)$ \\
2 & $\begin{array}{l}\text { GGAATATGATCTATTGAGATCATGGTGCGGAGTGCCTACTC + } \\
\text { GCTTCTAATCCACCAAGTACTTGG }\end{array}$ & N. fumigata promoter with easC extension $(809)$ \\
3 & $\begin{array}{l}\text { CTCGGAATTCGCTTCTAATCCACCAAGTACTTGG }+ \\
\text { GAGTACTAGTGTGGCCTTGAGTTAATCTGAAGC }\end{array}$ & N. fumigata promoter - easC fusion $(2,886)$ \\
4 & $\begin{array}{l}\text { GCCTGGAGCAAGTCAAGTTCTCA }+ \\
\text { TGATTCGACGATTCTCTCCACC }\end{array}$ & easC cDNA $(1,323)$, easC genomic DNA $(1,391)$ \\
\hline
\end{tabular}




\section{CHAPTER 5}

\section{SUMMARY}

Literature until now focused primarily on Periglandula sp. as ergot alkaloid producers and as endophytes of Ipomoea sp. Since Periglandula sp. cannot be grown in pure culture, DNA sequencing has been limited and genetic manipulation could not be conducted. This has so far prevented tracking of these fungal species through molecular means and has prevented the advancement of ergot alkaloid biosynthesis studies like those that have been conducted with other ergot alkaloid producing species. The first objective of this study was to investigate the impacts of Periglandula species infections on seed metabolite profiles of plants belonging to the genus Ipomoea. The second goal of this work was to investigate whether there are non-ergot alkaloid biomarkers of fungal presence in seed extracts. The results of the metabolomics data presented indicate that apart from ergot alkaloids, Periglandula species have a minimal impact on the metabolome of seeds of their host plants. We also found no evidence of non-ergot alkaloid producing Periglandula species in seeds of the Ipomoea species analyzed. This may suggest that, along with their plant embryo hosts, the Periglandula sp. endophytes are metabolically dormant at this stage.

One unexpected and novel observation from this study was the presence of the glycoside form of ergonovine. A major form of this compound, synthesized through plant vascular processes, is likely a galactoside but chromatography data suggest that there may be other hexose residue forms. That plants from a variety of clades produce this ergonovine glycoside suggests that plants such as Ipomoea sp. and Achnatherum sp. (which harbor ergonovine producing endophytes) have harnessed this process to gain a competitive advantage in their ecological niche. Of the ergot alkaloids described in Ipomoea species, only ergonovine was found to be present in a glycoside form. Given ergonovine's activity against 
invertebrates, more work could be done to evaluate the toxicity of this compound (Durden et al., 2019, Kaur et al., 2020). Perhaps plants harboring ergonovine-producing endophytes are using this form of the molecule to mobilize it throughout their tissues or a particularly potent toxin in their fight against invertebrates who would consume them.

Metarhizium spp. are known to harbor a wide range of specialized metabolites as virulence factors in their role as entomopathogens (Freimoser et al., 2003). With M. brunneum shown to produce ergot alkaloids (Leadmon et al., 2020), this was the first study to investigate the activity of these compounds as a virulence factor for this fungus. Additionally, the development of a hypervirulent ergot alkaloid deficient M. brunneum mutant (Tyo et al., 2018) provided a useful resource to test the effect of ergot alkaloids as virulence factors. One of the stark differences between the two strains of $M$. brunneum was that the ergot alkaloid deficient strain would not produce conidia after killing its insect host (Tyo et al., 2018). The results of protein expression analysis and of the melanization analysis point to ergot alkaloids as immune system suppressants in these infections. As an entomopathogen, $M$. brunneum is walking a narrow path of consuming its host, but not killing it too fast as to prevent creating an environment non-conducive to its own reproduction. As the hypervirulent strain of $M$. brunneum kills its host so fast that it cannot conidiate, it prevents its own spread which makes it a worse pathogen from an ecological perspective. An ergot alkaloid competent strain of M. brunneum is able to kill its host slowly enough that it does not prevent the conidiation process, which allows the next generation of $M$. brunneum spread to other insects, making it ultimately a more efficient pathogen.

The observation of ergot alkaloids in fungi infecting insects and significant roles for those ergot alkaloids is in stark contrast with the observations of $M$. brunneum growing in conjunction with a plant host, where it produced no ergot alkaloids (Leadmon et al., 2020). This contrast perhaps points to $M$. brunneum regularly growing alongside plants but changing its metabolism to infect insects when the opportunity presents itself. Indeed, the phylogeny of Clavicipitaceous fungi shows that the ancestral 
lifestyle for many of these fungi was as entomopathogens, and that some interactions with plants led to species adopting endophyte lifestyles (Spatafora et al., 2007). Given this perspective, it is possible that an ancestor of fungi like Epichloë spp. and Periglandula spp. accumulated enough mutations that they became poor entomopathogens and adapted to a strictly endophyte lifestyle. These interactions provided a benefit to their plant hosts whom they helped to protect from mammalian and insect herbivores. Furthermore, if Metarhizium brunneum symbiosis is an example, perhaps fungi promote metabolic shifts in plants which help the plant to tolerate other stressful conditions.

Ergot alkaloid synthesis likely does carry a cost for the fungus, especially considering that the synthetic pathways are inefficient and accumulate many intermediates. Apparently in the case of the domesticated fungus Penicillium camemberti this cost was prohibitive for the long-term maintenance of a pathway that was not needed. In the absence of competitors in the cheese making process, a mutation which inactivated the early step of the ergot alkaloid synthesis pathway could persist. This shows that there is some evolutionary benefit to species such as $P$. ipomoea and $M$. brunneum to maintain these inefficient pathways. As discussed in the introduction, an ergot different ergot alkaloid compounds have different activities against different organisms (e.g. mammals, insects, bacteria). Perhaps the advantage for these fungi to maintain such an inefficient pathway allows them to produce compounds with a central scaffold. This central scaffold can be modified in a way that gives the compounds activity in the wide range of circumstances the fungi find themselves in and help them or their symbionts to compete in their ecological niches. While this is not a new idea, it was proposed by Panaccione ( 2005), the new information provided in this dissertation help to provide evidence for that central concept regarding the ecological function of ergot alkaloids and their synthesis. 


\section{REFERENCES}

Baskys, A., \& Hou, A. C. (2007). Vascular dementia: pharmacological treatment approaches and perspectives. In Clinical interventions in aging.

Beaulieu, W. T. (2014). Chemical, ecological and co-evolutionary interactions between Periglandula and Convolvulaceae. Indiana University.

Beaulieu, W. T., Panaccione, D. G., Hazekamp, C. S., Mckee, M. C., Ryan, K. L., \& Clay, K. (2013). Differential Allocation of Seed-Borne Ergot Alkaloids During Early Ontogeny of Morning Glories (Convolvulaceae). Journal of Chemical Ecology, 39(7), 919-930. https://doi.org/10.1007/s10886013-0314-z

Beaulieu, W. T., Panaccione, D. G., Ryan, K. L., Kaonongbua, W., \& Clay, K. (2015). Phylogenetic and chemotypic diversity of Periglandula species in eight new morning glory hosts (Convolvulaceae). Mycologia, 107(4), 667-678. https://doi.org/10.3852/14-239

Belser-Ehrlich, S., Harper, A., Hussey, J., \& Hallock, R. (2013). Human and cattle ergotism since 1900: Symptoms, outbreaks, and regulations. In Toxicology and Industrial Health. https://doi.org/10.1177/0748233711432570

Betts, M. J., \& Russell, R. B. (2007). Amino-Acid Properties and Consequences of Substitutions. In Bioinformatics for Geneticists: A Bioinformatics Primer for the Analysis of Genetic Data: Second Edition. https://doi.org/10.1002/9780470059180.ch13

Boldo, J. T., Junges, A., do Amaral, K. B., Staats, C. C., Vainstein, M. H., \& Schrank, A. (2009). Endochitinase $\mathrm{CH} 2$ of the biocontrol fungus Metarhizium anisopliae affects its virulence toward the cotton stainer bug Dysdercus peruvianus. In Current Genetics. https://doi.org/10.1007/s00294009-0267-5

Bonuccelli, U., Del Dotto, P., \& Rascol, O. (2009). Role of dopamine receptor agonists in the treatment of early Parkinson's disease. Parkinsonism and Related Disorders. https://doi.org/10.1016/S13538020(09)70835-1

Brown, S. E., Howard, A., Kasprzak, A. B., Gordon, K. H., \& East, P. D. (2008). The discovery and analysis of a diverged family of novel antifungal moricin-like peptides in the wax moth Galleria mellonella. Insect Biochemistry and Molecular Biology, 38(2), 201-212. https://doi.org/10.1016/j.ibmb.2007.10.009

Buszewski, B., \& Noga, S. (2012). Hydrophilic interaction liquid chromatography (HILIC)-a powerful separation technique. Analytical and Bioanalytical Chemistry, 402(1), 231-247. https://doi.org/10.1007/s00216-011-5308-5

Cheeseman, K., Ropars, J., Renault, P., Dupont, J., Gouzy, J., Branca, A., Abraham, A. L., Ceppi, M., Conseiller, E., Debuchy, R., Malagnac, F., Goarin, A., Silar, P., Lacoste, S., Sallet, E., Bensimon, A., Giraud, T., \& Brygoo, Y. (2014). Multiple recent horizontal transfers of a large genomic region in cheese making fungi. Nature Communications. https://doi.org/10.1038/ncomms3876

Cooper, J. E. (2007). Early interactions between legumes and rhizobia: Disclosing complexity in a molecular dialogue. In Journal of Applied Microbiology. https://doi.org/10.1111/j.1365- 
2672.2007.03366.x

Craig, A. M., Klotz, J. L., \& Duringer, J. M. (2015). Cases of ergotism in livestock and associated ergot alkaloid concentrations in feed. Frontiers in Chemistry. https://doi.org/10.3389/fchem.2015.00008

Donnet, A., Braunstein, D., Pradel, V., Sciortino, V., Allaria-Lapierre, V., Micallef, J., \& Lanteri-Minet, M. (2016). Ergot Use and Overuse: A Pharmacoepidemiology Retrospective Cohort Study. Headache. https://doi.org/10.1111/head.12776

Duan, Z., Shang, Y., Gao, Q., Zheng, P., \& Wang, C. (2009). A phosphoketolase Mpk1 of bacterial origin is adaptively required for full virulence in the insect-pathogenic fungus Metarhizium anisopliae. Environmental Microbiology. https://doi.org/10.1111/j.1462-2920.2009.01961.x

Durden, L., Wang, D., Panaccione, D., \& Clay, K. (2019). Decreased Root-Knot Nematode Gall Formation in Roots of the Morning Glory Ipomoea tricolor Symbiotic with Ergot Alkaloid-Producing Fungal Periglandula Sp. Journal of Chemical Ecology, November. https://doi.org/10.1007/s10886-01901109-w

Eckart, E., \& Pertz, H. (1999). ANTIMICROBIAL AND ANTITUMOR EFFECTS OF ERGOT ALKALOIDS AND THEIR DERIVATIVES. In Ergot: the genus Claviceps (pp. 441-449).

Fabian, S. J., Maust, M. D., \& Panaccione, D. G. (2018). Ergot alkaloid synthesis capacity of Penicillium camemberti. Applied and Environmental Microbiology, 84(19), 1-14. https://doi.org/10.1128/AEM.01583-18

Faria, M. R. d., \& Wraight, S. P. (2007). Mycoinsecticides and Mycoacaricides: A comprehensive list with worldwide coverage and international classification of formulation types. Biological Control. https://doi.org/10.1016/j.biocontrol.2007.08.001

Flieger, M., Zelenkova, N. F., Sedmera, P., Kren, V., Novàk, J., Rylko, V., Sajdl, P., \& Rehacek, Z. (1989). Ergot alkaloid glycosides from saprophytic cultures of claviceps, I. Elymoclavine fructosides. Journal of Natural Products, 52(3), 506-510. https://doi.org/10.1021/np50063a007

Florea, S., Panaccione, D. G., \& Schardl, C. L. (2017). Ergot alkaloids of the family clavicipitaceae. Phytopathology, 107(5), 504-518. https://doi.org/10.1094/PHYTO-12-16-0435-RVW

Floss, H. G. (1976). Biosynthesis of ergot alkaloids and related compounds. Tetrahedron, 32(8), 873-912. https://doi.org/10.1016/0040-4020(76)85047-8

Gerhards, N., Neubauer, L., Tudzynski, P., \& Li, S. (2014). Biosynthetic Pathways of Ergot Alkaloids. Toxins, 6, 3281-3295. https://doi.org/10.3390/toxins6123281

Goetz, K. E., Coyle, C. M., Cheng, J. Z., Connor, S. E., \& Panaccione, D. G. (2011). Ergot cluster-encoded catalase is required for synthesis of chanoclavine-I in Aspergillus fumigatus. Current Genetics, 57(3), 201-211. https://doi.org/10.1007/s00294-011-0336-4

Haarmann, T., Rolke, Y., Giesbert, S., \& Tudzynski, P. (2009). Plant diseases that changed the world: Ergot: from witchcraft to biotechnology. Molecular Plant Pathology, 10(4), 563-577. https://doi.org/10.1111/j.1364-3703.2009.00548.x

Hara, S., \& Yamakawa, M. (1995). Moricin, a novel type of antibacterial peptide isolated from the silkworm, Bombyx mori. Journal of Biological Chemistry. https://doi.org/10.1074/jbc.270.50.29923

Howard, W. F., Mcfadden, P. R., \& Keettel, W. C. (1964). Oxytocic Drugs in Fourth Stage of Labor. JAMA: 
The Journal of the American Medical Association.

https://doi.org/10.1001/jama.1964.03070060021004

Hu, X., Xiao, G., Zheng, P., Shang, Y., Su, Y., Zhang, X., Liu, X., Zhan, S., St. Leger, R. J., \& Wang, C. (2014). Trajectory and genomic determinants of fungal-pathogen speciation and host adaptation.

Proceedings of the National Academy of Sciences of the United States of America. https://doi.org/10.1073/pnas.1412662111

Hutner, S. H., Provasoli, L., Schatz, A., \& Haskins, C. P. (1950). Some Approaches to the Study of the Role of Metals in the Metabolism of Microorganisms. Source: Proceedings of the American Philosophical Society.

Ichou, F., Schwarzenberg, A., Lesage, D., Alves, S., Junot, C., MacHuron-Mandard, X., \& Tabet, J. C. (2014). Comparison of the activation time effects and the internal energy distributions for the CID, PQD and HCD excitation modes. Journal of Mass Spectrometry, 49(6), 498-508.

https://doi.org/10.1002/jms.3365

Kaur, N., Cooper, W. R., Duringer, J. M., Badillo-Vargas, I. E., Esparza-Díaz, G., Rashed, A., \& Horton, D. R. (2018). Survival and development of potato psyllid (Hemiptera: Triozidae) on Convolvulaceae: Effects of a plant-fungus symbiosis (Periglandula). PLOS ONE. https://doi.org/10.1371/journal.pone.0201506

Kaur, N., Cooper, W. R., Duringer, J. M., Rashed, A., Badillo-Vargas, I. E., Esparza-Díaz, G., \& Horton, D. R. (2020). Mortality of Potato Psyllid (Hemiptera: Triozidae) on Host Clippings Inoculated With Ergot Alkaloids. Journal of Economic Entomology, 113(5), 2079-2085. https://doi.org/10.1093/jee/toaa144

Kind, T., Tsugawa, H., Cajka, T., Ma, Y., Lai, Z., Mehta, S. S., Wohlgemuth, G., Barupal, D. K., Showalter, M. R., Arita, M., \& Fiehn, O. (2018). Identification of small molecules using accurate mass MS/MS search. Mass Spectrometry Reviews, 37(4), 513-532. https://doi.org/10.1002/mas.21535

Klotz, J. L. (2015). Activities and effects of ergot alkaloids on livestock physiology and production. In Toxins. https://doi.org/10.3390/toxins7082801

Křen, V., Flieger, M., \& Sajdl, P. (1990). Glycosylation of ergot alkaloids by free and immobilized cells of Claviceps purpurea. Applied Microbiology and Biotechnology, 32(6), 645-650. https://doi.org/10.1007/BF00164733

Křen, V., Sedmera, P., Havlíček, V., \& Fišerová, A. (1992). Enzymatic galactosylation of ergot alkaloids. Tetrahedron Letters, 33(47), 7233-7236. https://doi.org/10.1016/S0040-4039(00)60881-X

Kucht, S., Groß, J., Hussein, Y., Grothe, T., Keller, U., Basar, S., König, W. A., Steiner, U., \& Leistner, E. (2004). Elimination of ergoline alkaloids following treatment of Ipomoea asarifolia (Convolvulaceae) with fungicides. Planta, 219(4), 619-625. https://doi.org/10.1007/s00425-0041261-2

Leadmon, C. E., Sampson, J. K., Maust, M. D., Macias, A. M., Rehner, S. A., Kasson, M. T., \& Panaccione, D. G. (2020). Several Metarhizium Species Produce Ergot Alkaloids in a Condition-Specific Manner. Applied and Environmental Microbiology, 86(14), 1-14. https://doi.org/10.1128/AEM.00373-20

Lessard, M. H., Viel, C., Boyle, B., St-Gelais, D., \& Labrie, S. (2014). Metatranscriptome analysis of fungal strains Penicillium camemberti and Geotrichum candidum reveal cheese matrix breakdown and potential development of sensory properties of ripened Camembert-type cheese. BMC Genomics. 
https://doi.org/10.1186/1471-2164-15-235

Lesur, A., \& Domon, B. (2015). Advances in high-resolution accurate mass spectrometry application to targeted proteomics. Proteomics, 15(5-6), 880-890. https://doi.org/10.1002/pmic.201400450

Li, Y., Fan, Y., Ma, Y., Zhang, Z., Yue, H., Wang, L., Li, J., \& Jiao, Y. (2017). Effects of Exogenous YAminobutyric Acid (GABA) on Photosynthesis and Antioxidant System in Pepper (Capsicum annuum L.) Seedlings Under Low Light Stress. Journal of Plant Growth Regulation. https://doi.org/10.1007/s00344-016-9652-8

Lorenz, N., Olšovská, J., Šulc, M., \& Tudzynski, P. (2010). Alkaloid cluster gene ccsA of the ergot fungus Claviceps purpurea encodes chanoclavine I synthase, a flavin adenine dinucleotide-containing oxidoreductase mediating the transformation of $\mathrm{N}$-methyl-dimethylallyltryptophan to chanoclavine I. Applied and Environmental Microbiology. https://doi.org/10.1128/AEM.00737-09

Mak, P., Zdybicka-Barabas, A., \& Cytryńska, M. (2010). A different repertoire of Galleria mellonella antimicrobial peptides in larvae challenged with bacteria and fungi. Developmental and Comparative Immunology, 34(10), 1129-1136. https://doi.org/10.1016/j.dci.2010.06.005

Mc Namara, L., Carolan, J. C., Griffin, C. T., Fitzpatrick, D., \& Kavanagh, K. (2017). The effect of entomopathogenic fungal culture filtrate on the immune response of the greater wax moth, Galleria mellonella. Journal of Insect Physiology, 100(February), 82-92. https://doi.org/10.1016/j.jinsphys.2017.05.009

Meurant, G. (1981). Roquefortines. In Handbook of Toxic Fungal Metabolites (pp. 528-544). https://doi.org/10.1016/c2009-0-03073-6

Nagy-Szakal, D., Barupal, D. K., Lee, B., Che, X., Williams, B. L., Kahn, E. J. R., Ukaigwe, J. E., Bateman, L., Klimas, N. G., Komaroff, A. L., Levine, S., Montoya, J. G., Peterson, D. L., Levin, B., Hornig, M., Fiehn, O., \& Lipkin, W. I. (2018). Insights into myalgic encephalomyelitis/chronic fatigue syndrome phenotypes through comprehensive metabolomics. Scientific Reports, 8(1), 1-11. https://doi.org/10.1038/s41598-018-28477-9

Nakhleh, J., El Moussawi, L., \& Osta, M. A. (2017). The Melanization Response in Insect Immunity. In Advances in Insect Physiology. https://doi.org/10.1016/bs.aiip.2016.11.002

Nisa, H., Kamili, A. N., Nawchoo, I. A., Shafi, S., Shameem, N., \& Bandh, S. A. (2015). Fungal endophytes as prolific source of phytochemicals and other bioactive natural products: A review. In Microbial Pathogenesis. https://doi.org/10.1016/j.micpath.2015.04.001

Panaccione, D. G. (2005). Origins and significance of ergot alkaloid diversity in fungi. FEMS Microbiology Letters, 251(1), 9-17. https://doi.org/10.1016/j.femsle.2005.07.039

Panaccione, D. G. (2011). Ergot Alkaloids. In Industrial Applications. https://doi.org/10.1007/978-3-64211458-8_9

Panaccione, D. G., \& Arnold, S. L. (2017). Ergot alkaloids contribute to virulence in an insect model of invasive aspergillosis. Scientific Reports. https://doi.org/10.1038/s41598-017-09107-2

Panaccione, D. G., Cipoletti, J. R., Sedlock, A. B., Blemings, K. P., Schardl, C. L., Machado, C., \& Seidel, G. E. (2006). Effects of ergot alkaloids on food preference and satiety in rabbits, as assessed with gene-knockout endophytes in perennial ryegrass (Lolium perenne). Journal of Agricultural and Food Chemistry. https://doi.org/10.1021/jf060626u 
Parish, J. A., McCann, M. A., Watson, R. H., Hoveland, C. S., Hawkins, L. L., Hill, N. S., \& Bouton, J. H. (2003). Use of nonergot alkaloid-producing endophytes for alleviating tall fescue toxicosis in sheep. Journal of Animal Science. https://doi.org/10.2527/2003.8151316x

Parish, J. A., McCann, M. A., Watson, R. H., Paiva, N. N., Hoveland, C. S., Parks, A. H., Upchurch, B. L., Hill, N. S., \& Bouton, J. H. (2003). Use of nonergot alkaloid-producing endophytes for alleviating tall fescue toxicosis in stocker cattle. Journal of Animal Science. https://doi.org/10.2527/2003.81112856x

Peregrín-Alvarez, J. M., Sanford, C., \& Parkinson, J. (2009). The conservation and evolutionary modularity of metabolism. Genome Biology. https://doi.org/10.1186/gb-2009-10-6-r63

Perez-Lloret, S., \& Rascol, O. (2010). Dopamine receptor agonists for the treatment of early or advanced Parkinsons disease. In CNS Drugs. https://doi.org/10.2165/11537810-000000000-00000

Potter, D. A., Tyler Stokes, J., Redmond, C. T., Schardl, C. L., \& Panaccione, D. G. (2008). Contribution of ergot alkaloids to suppression of a grass-feeding caterpillar assessed with gene knockout endophytes in perennial ryegrass. Entomologia Experimentalis et Applicata. https://doi.org/10.1111/j.1570-7458.2007.00650.x

Ramesh, S. A., Tyerman, S. D., Gilliham, M., \& Xu, B. (2017). Y-Aminobutyric acid (GABA) signalling in plants. In Cellular and Molecular Life Sciences (Vol. 74, Issue 9, pp. 1577-1603). https://doi.org/10.1007/s00018-016-2415-7

Robinson, S. L., \& Panaccione, D. G. (2015). Diversification of ergot alkaloids in natural and modified fungi. Toxins, 7(1), 201-218. https://doi.org/10.3390/toxins7010201

Ropars, J., Rodríguez De La Vega, R. C., López-Villavicencio, M., Gouzy, J., Sallet, E., Dumas, É., Lacoste, S., Debuchy, R., Dupont, J., Branca, A., \& Giraud, T. (2015). Adaptive horizontal gene transfers between multiple cheese-associated fungi. Current Biology. https://doi.org/10.1016/j.cub.2015.08.025

Ryan, K. L., Akhmedov, N. G., \& Panaccione, D. G. (2015). Identification and structural elucidation of ergotryptamine, a new ergot alkaloid produced by genetically modified aspergillus nidulans and natural isolates of epichlo?? species. Journal of Agricultural and Food Chemistry, 63(1), 61-67. https://doi.org/10.1021/jf505718x

Ryan, K. L., Moore, C. T., \& Panaccione, D. G. (2013). Partial reconstruction of the ergot alkaloid pathway by heterologous gene expression in Aspergillus nidulans. Toxins. https://doi.org/10.3390/toxins5020445

Schardl, C. L., Young, C. A., Hesse, U., Amyotte, S. G., Andreeva, K., Calie, P. J., Fleetwood, D. J., Haws, D. C., Moore, N., Oeser, B., Panaccione, D. G., Schweri, K. K., Voisey, C. R., Farman, M. L., Jaromczyk, J. W., Roe, B. A., O'Sullivan, D. M., Scott, B., Tudzynski, P., ... Zeng, Z. (2013). Plant-Symbiotic Fungi as Chemical Engineers: Multi-Genome Analysis of the Clavicipitaceae Reveals Dynamics of Alkaloid Loci. PLoS Genetics, 9(2). https://doi.org/10.1371/journal.pgen.1003323

Schrimpe-Rutledge, A. C., Codreanu, S. G., Sherrod, S. D., \& McLean, J. A. (2016). Untargeted Metabolomics Strategies-Challenges and Emerging Directions. Journal of the American Society for Mass Spectrometry. https://doi.org/10.1007/s13361-016-1469-y

Sheehan, G., \& Kavanagh, K. (2018). Analysis of the early cellular and humoral responses of galleria mellonella larvae to infection by Candida albicans. Virulence, 9(1), 163-172. 
https://doi.org/10.1080/21505594.2017.1370174

Shymanovich, T., Saari, S., Lovin, M. E., Jarmusch, A. K., Jarmusch, S. A., Musso, A. M., Charlton, N. D., Young, C. A., Cech, N. B., \& Faeth, S. H. (2014). Alkaloid Variation Among Epichloid Endophytes of Sleepygrass (Achnatherum robustum) and Consequences for Resistance to Insect Herbivores. Journal of Chemical Ecology, 41(1), 93-104. https://doi.org/10.1007/s10886-014-0534-x

Spatafora, J. W., Sung, G. H., Sung, J. M., Hywel-Jones, N. L., \& White, J. F. (2007). Phylogenetic evidence for an animal pathogen origin of ergot and the grass endophytes. Molecular Ecology, 16(8), 17011711. https://doi.org/10.1111/j.1365-294X.2007.03225.x

St. Leger, R. J., Joshi, L., Bidochka, M. J., \& Roberts, D. W. (1996). Construction of an improved mycoinsecticide overexpressing a toxic protease. Proceedings of the National Academy of Sciences of the United States of America. https://doi.org/10.1073/pnas.93.13.6349

St. Leger, R. J., Wang, C., \& Fang, W. (2011). New perspectives on insect pathogens. Fungal Biology Reviews, 25(2), 84-88. https://doi.org/10.1016/j.fbr.2011.04.005

Steiner, U., Leibner, S., Schardl, C. L., Leuchtmann, A., \& Leistner, E. (2011). Periglandula , a new fungal genus within the Clavicipitaceae and its association with Convolvulaceae. Mycologia, 103(5), 11331145. https://doi.org/10.3852/11-031

Steiner, U., \& Leistner, E. (2012). Ergoline alkaloids in convolvulaceous host plants originate from epibiotic clavicipitaceous fungi of the genus Periglandula. Fungal Ecology, 5(3), 316-321. https://doi.org/10.1016/j.funeco.2011.04.004

Tfelt-Hansen, P. C., \& Koehler, P. J. (2008). History of the use of ergotamine and dihydroergotamine in migraine from 1906 and onward. Cephalalgia. https://doi.org/10.1111/j.1468-2982.2008.01578.x

Tfelt-Hansen, Peer C. (2013). Triptans and ergot alkaloids in the acute treatment of migraine: similarities and differences. In Expert review of neurotherapeutics. https://doi.org/10.1586/14737175.2013.832851

Vijayakumari, K., \& Puthur, J. T. (2016). p-Aminobutyric acid (GABA) priming enhances the osmotic stress tolerance in Piper nigrum Linn. plants subjected to PEG-induced stress. Plant Growth Regulation. https://doi.org/10.1007/s10725-015-0074-6

Vogel, H., Altincicek, B., Glöckner, G., \& Vilcinskas, A. (2011). A comprehensive transcriptome and immune-gene repertoire of the lepidopteran model host Galleria mellonella. BMC Genomics, 12. https://doi.org/10.1186/1471-2164-12-308

Wallwey, C., \& Li, S.-M. (2011). Ergot alkaloids: structure diversity, biosynthetic gene clusters and functional proof of biosynthetic genes. Nat. Prod. Rep., 28(3), 496-510. https://doi.org/10.1039/CONP00060D

Wallwey, C., Matuschek, M., \& Li, S. M. (2010). Ergot alkaloid biosynthesis in Aspergillus fumigatus: Conversion of chanoclavine-I to chanoclavine-I aldehyde catalyzed by a short-chain alcohol dehydrogenase FgaDH. Archives of Microbiology, 192(2), 127-134. https://doi.org/10.1007/s00203-009-0536-1

Wang, B., Kang, Q., Lu, Y., Bai, L., \& Wang, C. (2012). Unveiling the biosynthetic puzzle of destruxins in Metarhizium species. Proceedings of the National Academy of Sciences of the United States of America. https://doi.org/10.1073/pnas.1115983109 
Wang, J., \& Hou, B. (2009). Glycosyltransferases: Key players involved in the modification of plant secondary metabolites. Frontiers of Biology in China, 4(1), 39-46. https://doi.org/10.1007/s11515008-0111-1

Xu, J., Liu, T., Yang, S., Jin, X., Qu, F., Huang, N., \& Hu, X. (2019). Polyamines are involved in GABAregulated salinity-alkalinity stress tolerance in muskmelon. Environmental and Experimental Botany. https://doi.org/10.1016/j.envexpbot.2019.05.011

Xu, X. X., Zhong, X., Yi, H. Y., \& Yu, X. Q. (2012). Manduca sexta gloverin binds microbial components and is active against bacteria and fungi. Developmental and Comparative Immunology, 38(2), 275-284. https://doi.org/10.1016/j.dci.2012.06.012

Yeung, P. K. (2018). Metabolomics and biomarkers for drug discovery. Metabolites, 8(1), 10-12. https://doi.org/10.3390/metabo8010011

Yi, H. Y., Chowdhury, M., Huang, Y. D., \& Yu, X. Q. (2014). Insect antimicrobial peptides and their applications. In Applied Microbiology and Biotechnology. https://doi.org/10.1007/s00253-0145792-6

Young, C. A., Schardl, C. L., Panaccione, D. G., Florea, S., Takach, J. E., Charlton, N. D., Moore, N., Webb, J. S., \& Jaromczyk, J. (2015). Genetics, genomics and evolution of ergot alkaloid diversity. In Toxins (Vol. 7, Issue 4, pp. 1273-1302). https://doi.org/10.3390/toxins7041273

Yu, J., Schorlemer, M., Gomez Toledo, A., Pett, C., Sihlbom, C., Larson, G., Westerlind, U., \& Nilsson, J. (2016). Distinctive MS/MS Fragmentation Pathways of Glycopeptide-Generated Oxonium lons Provide Evidence of the Glycan Structure. Chemistry - A European Journal, 22(3), 1114-1124. https://doi.org/10.1002/chem.201503659 